\title{
Reflectance and Texture of Real-World Surfaces
}

\author{
Columbia University Technical Report \\ CUCS-048-96 \\ December 1996
}

Kristin J. Dana Shree K. Nayar

Department of Computer Science

Columbia University

New York, NY 10027

Email:dana@cs.columbia.edu
Bram van Ginneken

Jan J. Koenderink

Department of Physics

Utrecht University

3508 TA Utrecht, the Netherlands

Email: Bram.vanGinneken@cv.ruu.nl 


\title{
Reflectance and Texture of Real World Surfaces
}

\begin{abstract}
In this work, we investigate the visual appearance of real-world surfaces and the dependence of appearance on scale, viewing direction and illumination direction. At fine scale, surface variations cause local intensity variation or image texture. The appearance of this texture depends on both illumination and viewing direction and can be characterized by the BTF (bidirectional texture function). At sufficiently coarse scale, local image texture is not resolvable and local image intensity is uniform. The dependence of this image intensity on illumination and viewing direction is described by the BRDF (bidirectional reflectance distribution function). We simultaneously measure the BTF and BRDF of over 60 different rough surfaces, each observed with over 200 different combinations of viewing and illumination direction. The resulting BTF database is comprised of over 12,000 image textures. To enable convenient use of the BRDF measurements, we fit the measurements to two recent models and obtain a BRDF parameter database. These parameters can be used directly in image analysis and synthesis of a wide variety of surfaces. The BTF, BRDF, and BRDF parameter databases have important implications for computer vision and computer graphics and and each is made publicly available.
\end{abstract}




\section{INTRODUCTION}

Texture has long been an important and challenging topic in computer vision and computer graphics. Unlike the tangible quantities of shape or color, texture is a more abstract term encompassing both random and deterministic variations of both albedo and surface height. Because of the numerous meanings of the word texture, it is useful to explicitly identify the taxonomy of texture used in this work. We concentrate on texture due to surface height variation which we term 3D-texture, to be contrasted with albedo or color variation which we term 2D-texture. Examples of 3D-textured surfaces encountered in practice include: foliage, soil, and sand in natural scenes; concrete, brick and pavement in urban scenes; rugs, upholstery and textured walls in domestic scenes; hair, skin, and clothing of people in portrait scenes.

Characterizing the appearance of real-world textured surfaces is a fundamental problem in computer vision and computer graphics. Appearance depends on view, illumination and the scale at which the texture is observed. At coarse scale, where local surface variations are subpixel and local intensity is uniform, appearance is characterized by the BRDF (bidirectional reflectance distribution function). At fine scale, where the surface variations give rise to local intensity variation, appearance can be characterized by the BTF (bidirectional texture function). As a direct analogy to the BRDF, we introduce the term BTF to describe the appearance of texture as a function of illumination and viewing direction. This taxonomy is illustrated in Figure 1 and Figure 2.

Our investigation of the reflectance and texture of real-world surfaces has resulted in three publicly available databases: a BTF measurement database with texture images from over 60 different samples, each observed with over 200 combinations of viewing and illumination directions, a BRDF measurement database with reflectance measurements from the same 60 samples and a BRDF model parameter database with parameters obtained by fitting the measured BRDF data to two recent BRDF models. Each of these databases is made publicly available at http://www.cs.columbia.edu/CAVE/curet.

Although BRDF models have been widely discussed and used in vision (see [13],[24],[29],[9],[15]) the BRDFs of a large and diverse collection of macroscopically rough, real-world surfaces have never before been obtained ${ }^{1}$. Our measurement procedure does not employ a gonioreflectometer or the hemispherical mirror arrangement described in [27]. Instead, a robotic manipulator and CCD camera are employed to allow simultaneous measurement of the BTF and the BRDF of large samples (about 10x10cm). Our BRDF measurements comprise a comprehensive database (the first of its kind) for macroscopically rough surfaces that is now publicly available. Exactly how well the BRDFs of real-world surfaces fit existing models has remained unknown as each model is typically verified using a small number (2 to 6$)$ of surfaces. Our large database allows us to evaluate the performance of known models. Specifically, the measurements are fit to two existing analytical representations: the Oren-Nayar model [15] for surfaces with isotropic roughness and the Koenderink et al. decomposition [9] for both anisotropic and isotropic surfaces. Our fitting results form a concise BRDF parameter database that is also publicly available. These BRDF parameters can be directly used for both image analysis and image synthesis. In addition, the

\footnotetext{
${ }^{1}$ In the microscopic domain, measurements and models of the BRDF have had a longstanding role in scientific disciplines concerned with precise and detailed characterization of microscopically rough surfaces. In material science, BRDF measurements have been used to quantify microsurface topography, interfacial roughness, performance of optical coatings, etc. Specific applications of these measurements include quantifying surface roughness in epitaxial semiconductor multilayer structures, testing reflectivity of thermal coatings of used in space vehicles for thermal control analysis, controlling contamination by comparing BRDF's of clean and potentially contaminated samples, testing antireflective coating of components in an optical system, and predicting stray light due to surface scattering in optical systems.
} 
BRDF measurements can be used to evaluate other existing models [13],[24],[29] as well as future models.

While obtaining BRDF measurements, images of each real-world sample are recorded. These images prove valuable since they comprise a texture database, or a BTF database, with over 12,000 images (61 samples with 205 images per sample). Current literature deals almost exclusively with textures due to albedo and color variations on planar surfaces (see [26],[4],[8]). In contrast, the texture due to surface roughness has complex dependencies on viewing and illumination directions. These dependencies cannot be studied using existing texture databases that include few images (often a single image) of each sample (for instance, the widely used the Brodatz database). Our texture database covers a diverse collection of rough surfaces and captures the variation of image texture with changing illumination and viewing directions.

This BTF database can also be used for development of 3D texture rendering algorithms. Using these images, we demonstrate 3D texture mapping of real-world surfaces which shows a substantial improvement in realism over standard 2D texture mapping methods. Traditional methods of 2D texture mapping make no provision for the change in texture appearance with viewing and illumination directions. When a single digital image of a rough surface is mapped onto a 3D object, the appearance of roughness is usually lost or distorted. Bump-mapping preserves some of the appearance of roughness, but knowledge of the surface shape is required and shadows from the local surface relief are not rendered. Many of the problems associated with traditional texture mapping and bump-mapping techniques are described in [10]. If the exact geometry of the surface is known, ray tracing can be used but at a high computational cost. Other methods of rendering surface texture include cellular texturing [5] which models the surface geometry with synthesized cells, and solid texturing ([17],[19],[18],[12],[22]) which combines a volumetric texture synthesis with volume rendering techniques. Both of these methods are also computationally intensive and both methods are applicable for a limited variety of textures. With the availability of the BTF database, the potential exists for developing 3D texturing algorithms using image texture appearance without the need for a 3D surface model.

The measurements and model fitting results of this work are pertinent to a variety of areas including remote-sensing, photogrammetry, image understanding and scene rendering. The results are accordingly of interest to both the computer vision and computer graphics communities.

\section{METHODS}

\subsection{MEASUREMENT DEVICE}

Our measurement equipment depicted in Figure 3 is comprised of a $\operatorname{robot}^{2}, \operatorname{lamp}^{3}$, personal computer ${ }^{4}$, spectrometer ${ }^{5}$ and video camera ${ }^{6}$. Measuring the BRDF requires radiance measurements for a range of viewing/illumination directions. For each sample and each combination of illumination and viewing directions, an image from the video camera is captured by the frame grabber. These images have $640 \times 480$ pixels with 24 bits per pixel ( 8 bits per R/G/B channel). The pixel values are converted to radiance values using a post-processing calibration and segmentation scheme described in Appendix C and Appendix B. The calibrated, segmented images serve as the BTF measurements

\footnotetext{
${ }^{2}$ SCORBOT-ER V by ESHED Robotec (Tel Aviv, Israel).

${ }^{3}$ Halogen bulb with a Fresnel lens.

${ }^{4}$ IBM compatible PC running Windows 3.1 with "Videomaker" frame grabber by VITEC Multimedia.

${ }^{5}$ SpectraScan PR-704 by Photoresearch (Chatsworth,CA).

${ }^{6}$ Sony DXC-930 3-CCD color video camera.
} 


\section{Surface Appearance}

\begin{tabular}{|c|c|c|}
\hline & Coarse-Scale & Fine-Scale \\
\hline $\begin{array}{c}\text { Fixed } \\
\text { View / Illumination }\end{array}$ & Reflectance & Texture \\
\hline $\begin{array}{c}\text { Varied } \\
\text { View / Illumination }\end{array}$ & BRDF & BTF \\
\hline
\end{tabular}

Figure 1: Taxonomy of surface appearance. When viewing and illumination directions are fixed, surface appearance can be described by either reflectance (at coarse-scale observation) or texture (at fine-scale observation). When viewing and illumination directions vary, the equivalent descriptions are the bidirectional reflectance distribution function (BRDF) and the bidirectional texture function (BTF). Analogous to the BRDF, BTF is a function of four independent angles (two each for viewing and illumination directions). Note that for a sufficiently large surface area, the BRDF remains the same as the scale of observation becomes more coarse; however, the BTF can change as the scale of observation becomes more fine.

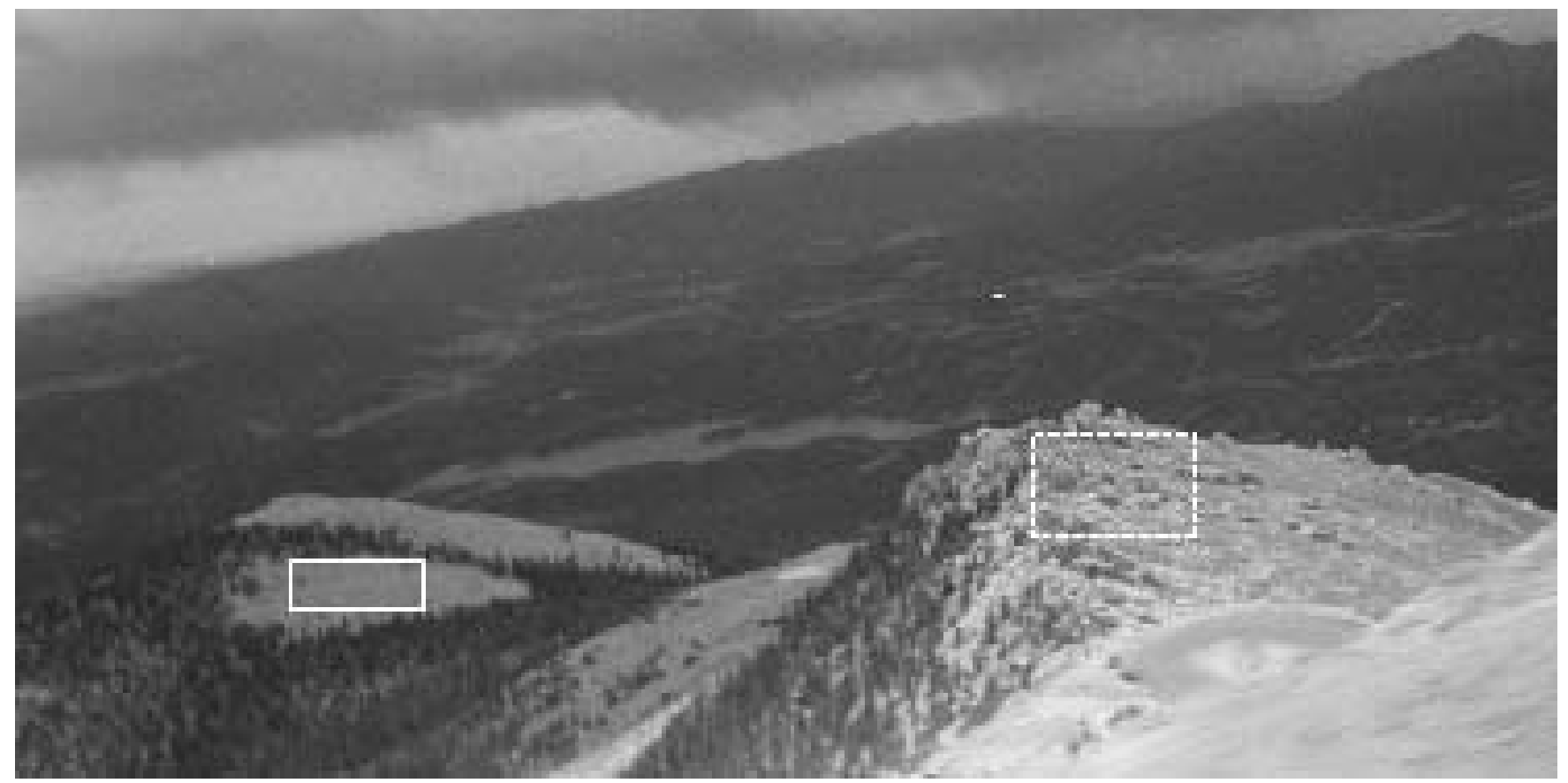

Figure 2: A View From Pike's Peak. Using the taxonomy of Figure 1, the appearance of the distantly viewed terrain in the solid-line square is characterized by the BRDF. The appearance of the closely viewed terrain in the dashed-line square is characterized by the BTF. 


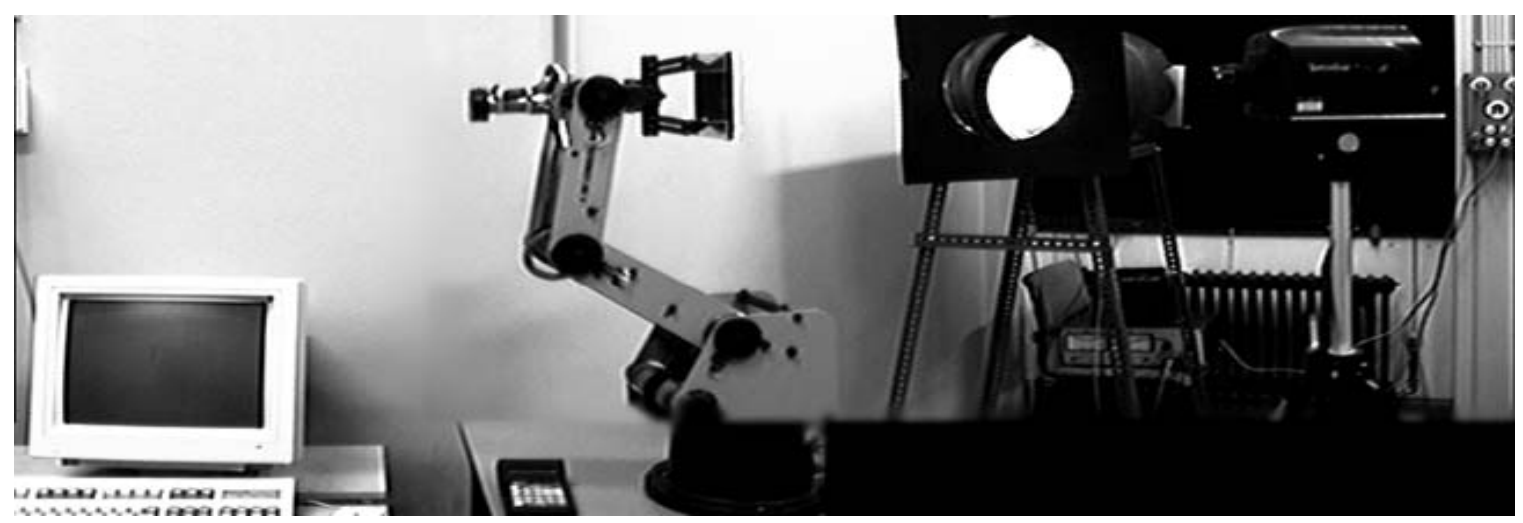

Figure 3: Measurement setup. The equipment consists of a personal computer with a 24-bit R/G/B frame grabber, a robot arm to orient the texture samples, a halogen bulb with a Fresnel lens which produces a parallel beam, a spectrometer and a 3-CCD color video camera (not shown).

and these images are averaged to obtain the BRDF measurements, as illustrated in Figure 4.

The need to vary the viewing and source directions over the entire hemisphere of possible directions presents a practical obstacle in the measurements. This difficulty is reduced considerably by orienting the sample to generate the varied conditions. As illustrated in Figure 5, the light source remains fixed throughout the measurements. The light rays incident on the sample are approximately parallel and uniformly illuminate the sample. The camera is mounted on a tripod and its optical axis is parallel to the floor of the lab. During measurements for a given sample, the camera is moved to seven different locations, each separated by 22.5 degrees in the ground plane at a distance of $200 \mathrm{~cm}$ from the sample. For each camera position, the sample is oriented so that its normal is directed toward the vertices of the facets which tessellate the fixed quarter-sphere illustrated in Figure 5. 7 With this arrangement, a considerable number of measurements are made in the plane of incidence (i.e. source direction, viewing direction and sample normal lie in the same plane). Also, for each camera position, a specular point is included where the sample normal bisects the angle between the viewing and source direction. Sample orientations with corresponding viewing angles or illumination angles greater than 85 degrees are excluded from the measurements to avoid self-occlusion and self-shadowing. This exclusion results in the collection of 205 images for each sample with 55 images taken at camera position 1, 48 images at position 2, 39 images at position 3, 28 images at position 4, 19 images at positions 5, 12 images at position 6 and 4 images at position 7. A complete table of sample orientations for each camera position is given in Appendix A.

Figure 6 shows an alternative representation of the 205 measurements for each sample. Here, the illumination directions are shown in the sample coordinate frame $\mathbf{x}_{\mathbf{s}}-\mathbf{y}_{\mathbf{s}}-\mathbf{z}_{\mathbf{s}}$. Notice that the illumination directions are evenly distributed over the quarter-sphere so the set of all possible illumination directions is well-represented by the subset used in the measurements. To determine which viewing directions are paired with each of these illumination directions, consider that each distinct illumination direction corresponds to a distinct sample orientation. Consequently, there are a maximum of 7 viewing directions for each illumination direction, corresponding to the 7 camera positions. There can be less than 7 viewing directions for a given illumination direction because a particular combination of sample orientation and camera position is excluded if the corresponding viewing direction is greater than 85 degrees from the sample normal. Figure 7 shows examples of

\footnotetext{
${ }^{7}$ The vertices of the quarter sphere shown in Figure 5(a) were obtained by starting with the two triangles formed by the coordinates $(1,0,0),(0,0,1),(0,-1,0)$, and $(0,1,0)$ and then barycentrically subdividing three times.
} 


\section{texture / BTF}

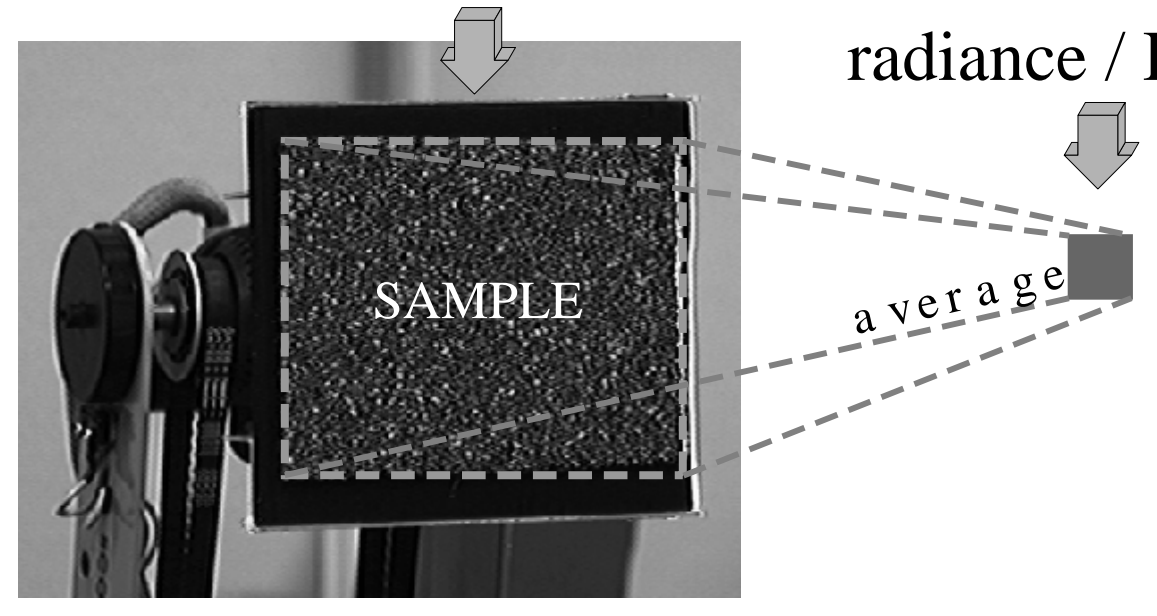

Figure 4: Sample (roofing shingle) mounted on a robot. The average intensity over the sample is the radiance or BRDF measurement and the image of the sample is the texture or BTF measurement for a particular viewing and source direction.

illumination directions with their associated viewing directions. From this figure it is readily seen that several measurements are taken in the plane of incidence and the specular position is always included.

For anisotropic textures, the 205 measurements are repeated after rotating the sample about $\mathbf{z}_{\mathbf{s}}$ by either 90 degrees or 45 degrees depending on the structure of the anisotropy. For example, a linen sample was rotated by 45 degrees and because it's horizontal and vertical threadings formed a gridlike structure that does not change when rotated by 90 degrees. On the other hand, the sample of corduroy was rotated by 90 degrees to obtain the maximum difference in surface structure.

\subsection{SAMPLES FOR MEASUREMENTS}

The collection of real-world surfaces used in the measurements are illustrated in Figure 8. Samples of these surfaces were mounted on $10 \times 12 \mathrm{~cm}$ bases which were constructed to fit onto the robot gripper. Each sample, though globally planar, exhibits considerable depth variation or macroscopic surface roughness. The samples were chosen to span a wide range of geometric and photometric properties. The categories include specular surfaces (aluminum foil, artificial grass), diffuse surfaces (plaster, concrete), isotropic surfaces (cork, leather, styrofoam), anisotropic surfaces (straw, corduroy, corn husk), surfaces with large height variations (crumpled paper, terrycloth, pebbles), surfaces with small height variations (sandpaper, quarry tile, brick), pastel surfaces (paper, cotton), colored surfaces (velvet, rug), natural surfaces (moss, lettuce, fur) and man-made surfaces (sponge, terrycloth, velvet). 


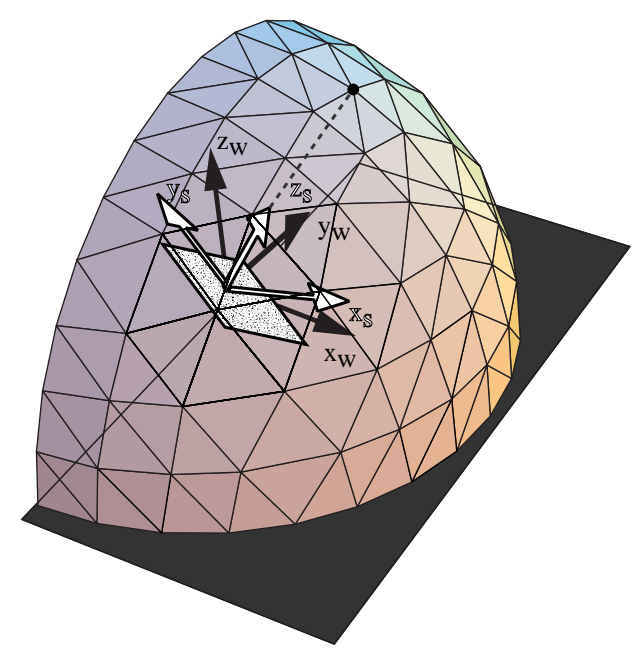

(a)

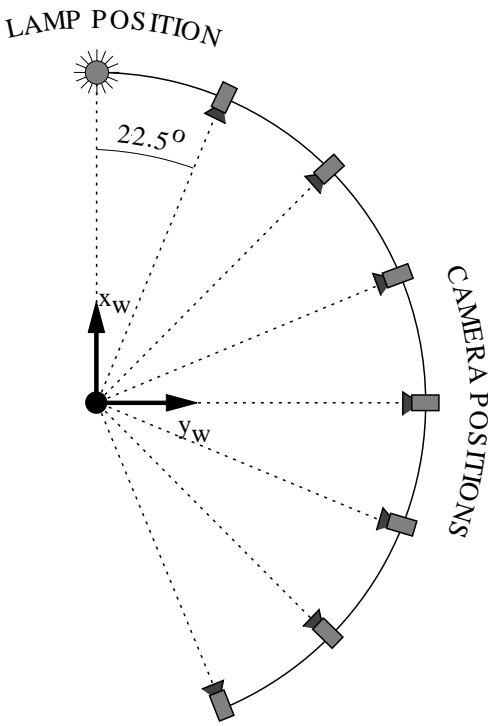

(b)

Figure 5: (a) Sample orientations. The vertices on the hemisphere correspond to the directions in which the robot orients the sample's surface normal $\left(\mathbf{z}_{\mathbf{s}}\right)$. The world coordinate frame $\mathbf{x}_{\mathbf{w}}-\mathbf{y}_{\mathbf{w}}-\mathbf{z}_{\mathrm{w}}$ is fixed with $\mathbf{x}_{\mathrm{w}}-\mathbf{y}_{\mathbf{w}}$ aligned with the floor and $\mathbf{z}_{\mathrm{w}}$ directed toward the ceiling. The sample coordinate frame $\mathbf{x}_{\mathbf{s}}-\mathbf{y}_{\mathbf{s}}-\mathbf{z}_{\mathbf{s}}$ is attached to the sample; $\mathbf{z}_{\mathbf{s}}$ is aligned with the sample normal while $\mathbf{x}_{\mathbf{s}}$ and $\mathbf{y}_{\mathbf{s}}$ are aligned with the horizontal and vertical edges of the sample. (b) Light source and camera positions. The illumination direction is fixed along $\mathbf{x}_{\mathrm{w}}$ and the camera is positioned at seven different locations in the $\mathbf{x}_{\mathbf{w}}-\mathbf{y}_{\mathbf{w}}$ plane. These seven locations correspond to angular deviations of $22.5^{\circ}, 45^{\circ}, 67.5^{\circ}$, $90^{\circ}, 112.5^{\circ}, 135^{\circ}, 157.5^{\circ}$ from $\mathbf{x}_{\mathrm{w}}$. For each camera position, images of the sample are acquired at the subset of sample orientations in (a) which are visible and illuminated. The total number of images acquired per sample is 205 . 


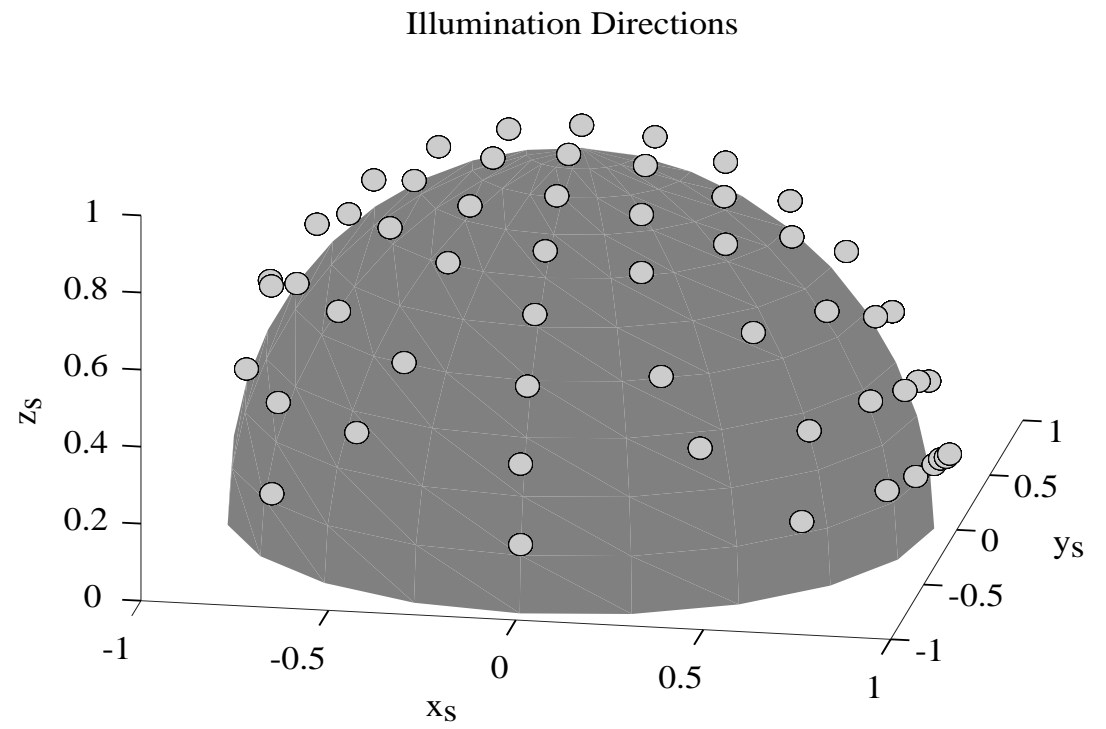

Figure 6: An alternative interpretation of the measurements described in Figure 5. Illumination directions are shown in the sample coordinate frame. The sample lies in the $\mathbf{x}_{\mathbf{s}}-\mathbf{y}_{\mathbf{s}}$ plane and its global normal points in the direction of $\mathbf{z}_{\mathbf{s}}$. Each circular marker represents a distinct illumination direction. For each of these illumination directions, the sample is imaged from 7 viewing directions, corresponding to the 7 camera positions shown in Figure 5 (b).
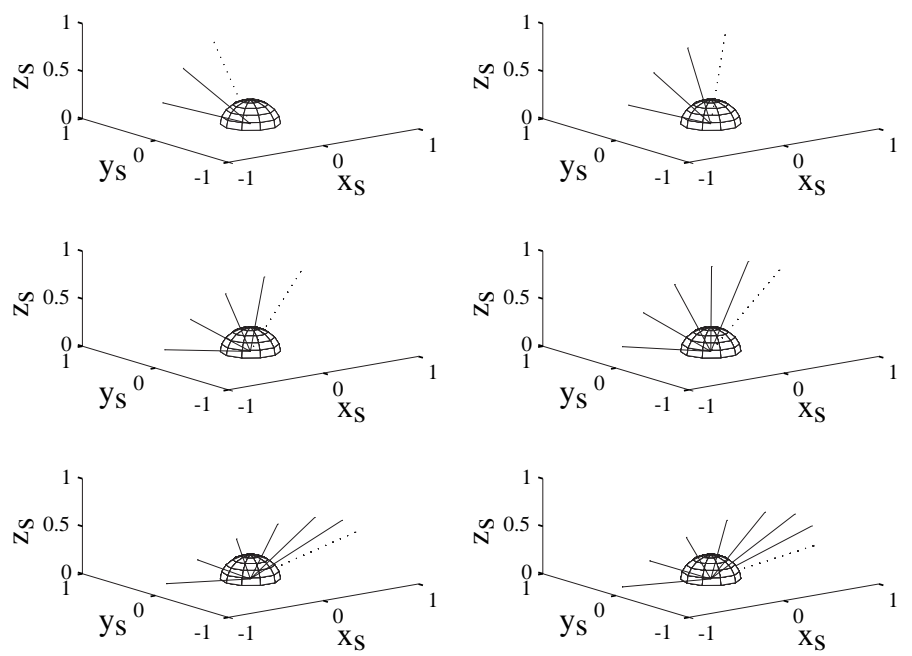

Figure 7: Viewing directions in the sample coordinate frame. For each illumination direction shown in Figure 6, there are at most seven associated viewing directions corresponding to the seven camera positions. Each panel shows an example of an illumination direction (dotted line) and the associated viewing directions (solid lines). The viewing directions lie in a plane that changes with illumination direction. Since the sample is not visible for every camera position, the number of associated viewing conditions also varies with illumination direction. The plots show examples of illumination directions with $2,3,4,5,6$, and 7 associated viewing directions. 


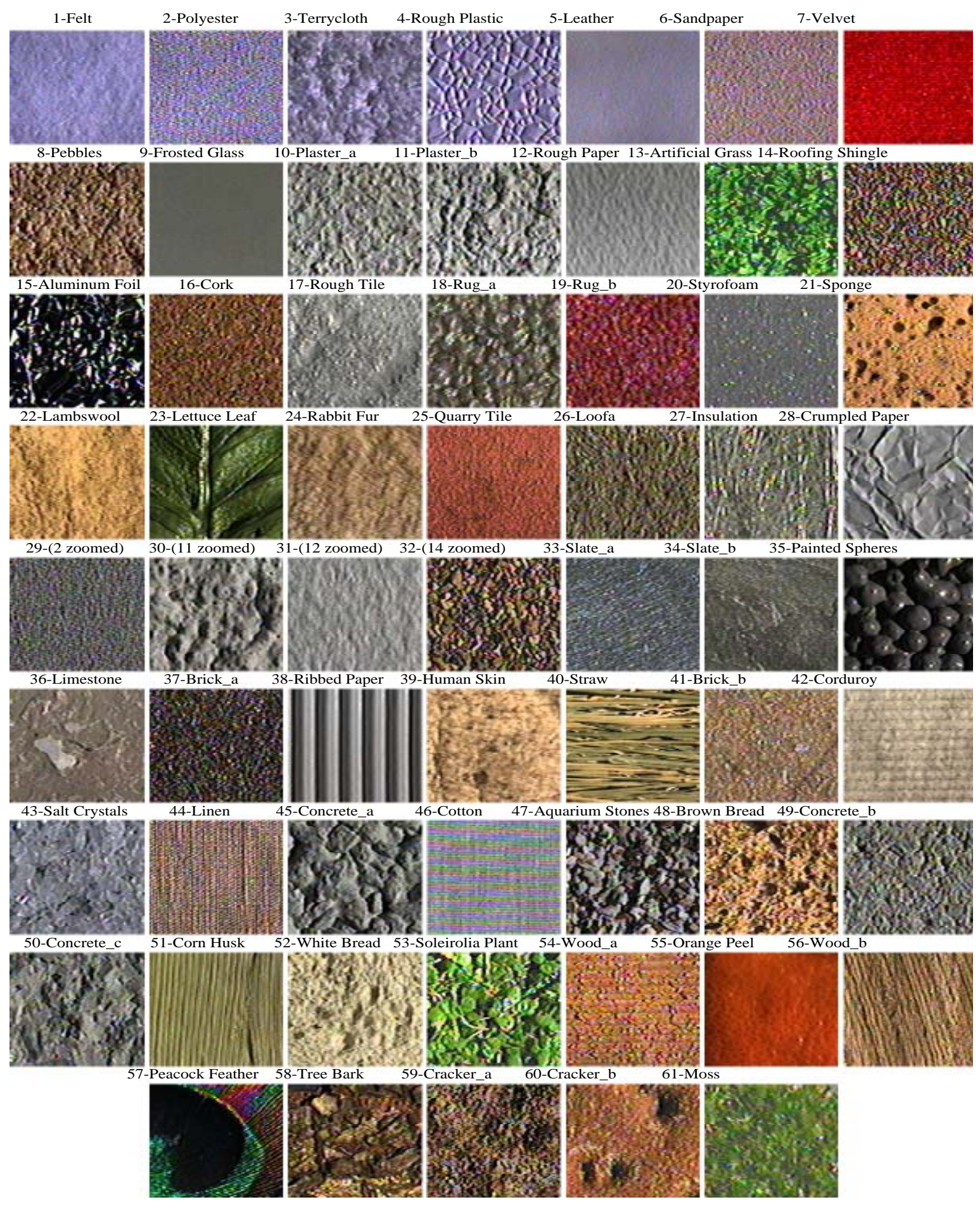

Figure 8: The collection of 61 real-world surfaces used in the measurements. The name and number of each sample is indicated above its image. The samples were chosen to span a wide range of geometric and photometric properties. The categories include specular surfaces (aluminum foil, artificial grass), diffuse surfaces (plaster, concrete), isotropic surfaces (cork, leather, styrofoam), anisotropic surfaces (straw, corduroy, corn husk), surfaces with large height variations (crumpled paper, terrycloth, pebbles), surfaces with small height variations (sandpaper, quarry tile, brick), pastel surfaces (paper, cotton), colored surfaces (velvet, rug), natural surfaces (moss, lettuce, fur) and man-made surfaces (sponge, terrycloth, velvet). Different samples of the same type of surfaces are denoted by letters, e.g. Brick_a and Brick_b. Samples $29,30,31$ and 32 are close-up views of samples $2,11,12$ and 14, respectively. 


\section{BRDF Database}

The BRDF measurements form a database with over 12,000 reflectance measurements (61 samples, 205 measurements per sample, 205 additional measurements for anisotropic samples). This entire set is available electronically at http://www.cs.columbia.edu/CAVE/curet. The measured BRDFs are quite diverse and reveal the complex appearance of many ordinary surfaces.

Figure 9 illustrates examples of spheres rendered with the measured BRDF as seen from camera position 1, i.e. with the illumination coming from $22.5^{\circ}$ to the right. Interpolation is used to obtain a continuous radiance pattern over each sphere. The rendered sphere corresponding to velvet shows a particularly interesting BRDF that has bright regions when the global surface normal is close to 90 degrees from the source direction. This effect can be accounted for by considering the individual strands comprising the velvet structure which reflect light strongly as the illumination becomes oblique. This effect is consistent with the observed brightness in the interiors of folds of a velvet sheet. Indeed, the rendered velvet sphere gives a convincing impression of velvet.

The rendered sphere of plaster shows a fairly flat appearance which is quite different from the Lambertian prediction for such matte objects, but is consistent with [14] and [15]. Sample 35, a collection of spheres painted with highly specular paint, show a similar flatness due to surface roughness. Compare sample 35 with sample 25 (quarry tile) which is a smoother sample that is only slightly specular. The rendered sphere from the measurements of sample 25 shows a clear specular lobe, while the rendered sphere from sample 35 is quite flat. This observation is counterintuitive since the material of sample 25 is significantly less specular than that of sample 35 . The observation can be explained by considering the geometries of the two surfaces. Because sample 35 is quite rough, the number of local surface points oriented in the specular direction is approximately the same for every global surface normal. The net result is a flat appearance for this highly specular, rough sample.

Rendered spheres from two anisotropic samples (sample 54 and sample 56) are also illustrated in Figure 9. The structure of the anisotropy of sample 54 consists of horizontally oriented ridges. This ridge structure causes a vertical bright stripe instead of a specular lobe in the rendered sphere. Sample 56 shows a similar effect, but the anisotropic structure for this sample consists of near vertical ridges. Consequently the corresponding rendered sphere shows a horizontal bright stripe due to specularities.

\section{Texture Database}

The appearance of a rough surface, whether manifested as a single radiance value or as image texture, depends on viewing and source direction. Just as the BRDF describes the coarse-scale appearance of a rough surface, the BTF (bidirectional texture function) is useful for describing the fine-scale appearance of a rough surface. Our measurements of image texture comprise the first BTF database for real-world surfaces. The database has over 12,000 images (61 samples, 205 measurements per sample, 205 additional measurements for anisotropic samples) and is available at http://www.cs.columbia.edu/CAVE/curet.

Important observations on the BTF can be made from the database. Consider the image texture of plaster (sample 11). The appearance of this sample from an oblique viewing direction is shown in Figure 10. A simulated oblique view is generated by mapping another image of plaster (frontal view) onto an appropriately tilted surface. This simulated view is also shown in Figure 10. The differences in the simulated and actual oblique views are readily apparent. Because of the varying surface

normals across the sample, foreshortening effects are quite complicated and cannot be accounted 


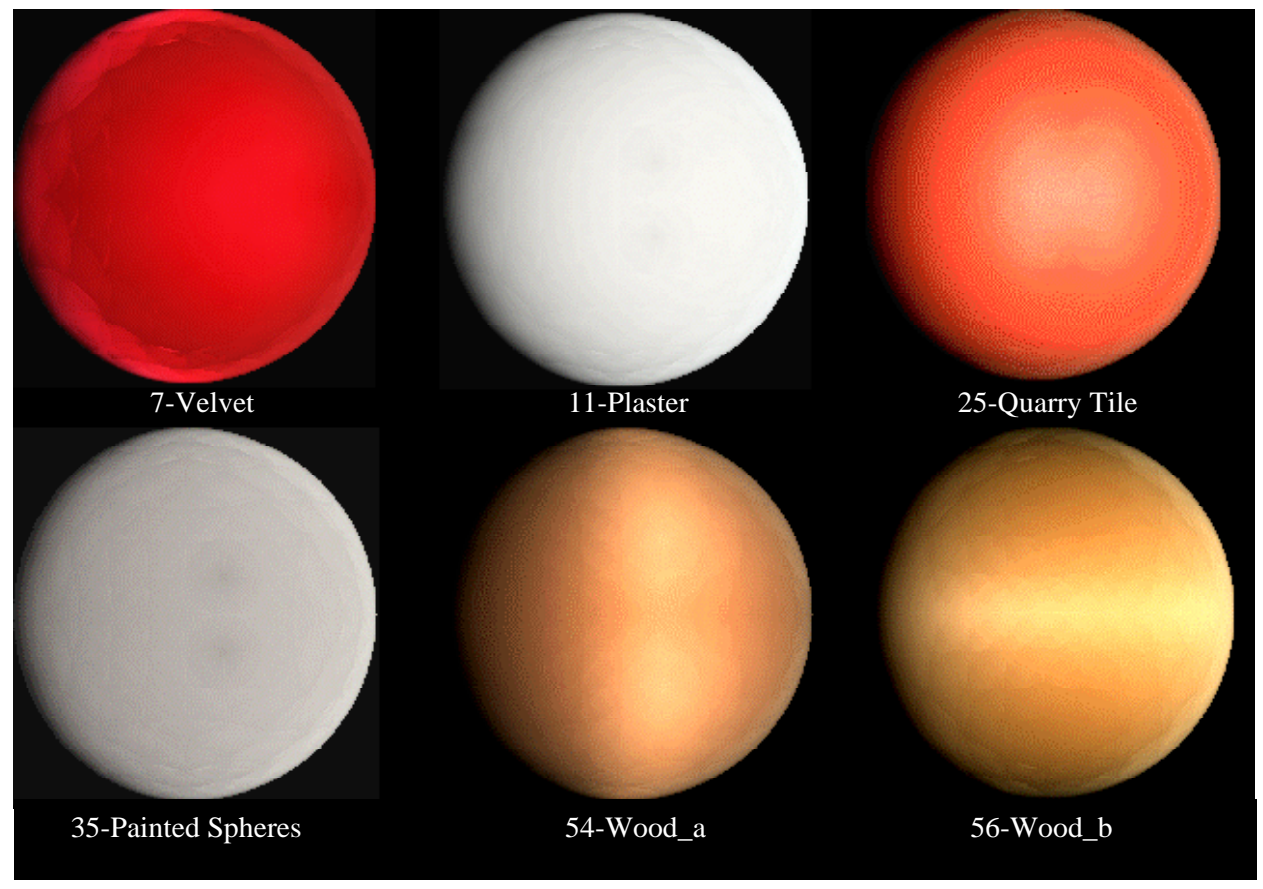

Figure 9: Spheres rendered from the BRDF measurements obtained from camera position 1. Interpolation was used to get radiance values between the measured positions.
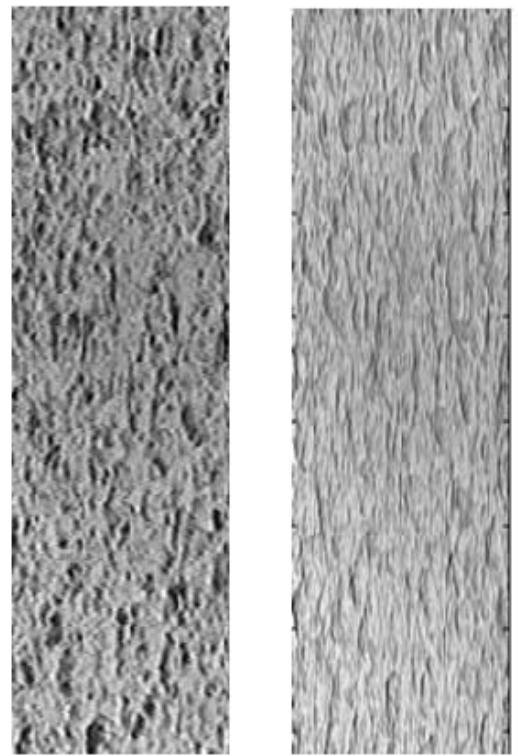

Figure 10: Obliquely viewed sample of plaster (left). Simulation of an obliquely viewed sample of plaster (right). The simulated oblique view is obtained by mapping an image of plaster (frontal view) onto a tilted surface. The clear differences in the left and right images show the importance of accounting for the surface structure. 

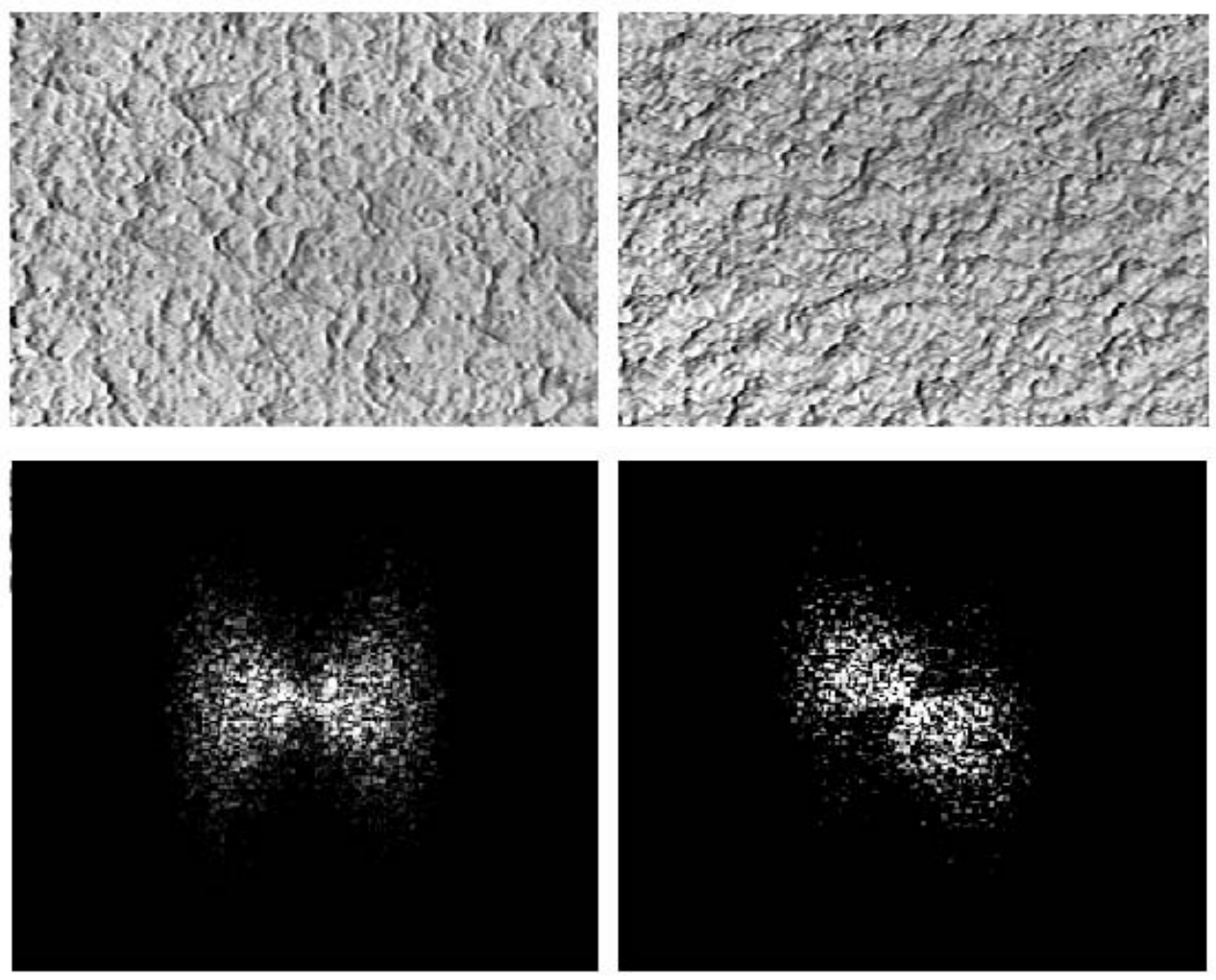

Figure 11: Changes in the spectrum due to changes in imaging conditions. (Top row) Two images of sample 11 with different source and viewing directions. (Bottom row) Fourier spectrum of the images in the top row, with zero frequency at the center and brighter regions corresponding to higher magnitudes. The orientation change in the spectrum is due to the change of source direction which causes a change in the shadow direction.

for by common texture-mapping techniques. A detailed discussion of the pitfalls of current texture rendering schemes is given in [10].

Consider the same sample shown under two different sets of illumination and viewing directions in Figure 11. The corresponding Fourier spectra are also shown in Figure 11. Notice that the spectra are quite different. Most of the difference is due to the change in azimuthal angle of the source direction which causes a change in the shadowing direction and hence a change in the dominant orientation of the spectrum. If the image texture was due to a planar albedo or color variation, changes in the source direction would not have this type of effect on the spectrum. Source direction changes would only cause a uniform scaling of the intensity over the entire image.

To illustrate the use of the BTF representation, Figure 12 and Figure 13 show rendered cylinders of plaster, pebbles, concrete, crumpled paper, plush rug, and rough wood using two methods: ordinary 2D texture mapping and 3D texture mapping using the BTF measurement. For 2D texture mapping, the texture is taken as the frontally viewed image with the illumination direction at an angle of $22.5^{\circ}$ to the right. For 3D texture mapping, 13 images per sample are used from the database collection of 205 images. Of these 13 images, one view is the frontal view and the rest are oblique views of the sample. More specifically, these 13 images correspond to orientations of the sample's global surface normal in the plane of the viewing and illumination direction at intervals of 11.25 degrees as shown in Figure 5. We make a piece-wise planar approximation of the cylinder so that each planar section corresponds to the viewing and illumination direction of one of the 13 images. Then a section of each image is pasted in its appropriate position with blurring of 3 pixels at the section borders to reduce the appearance of seams. 

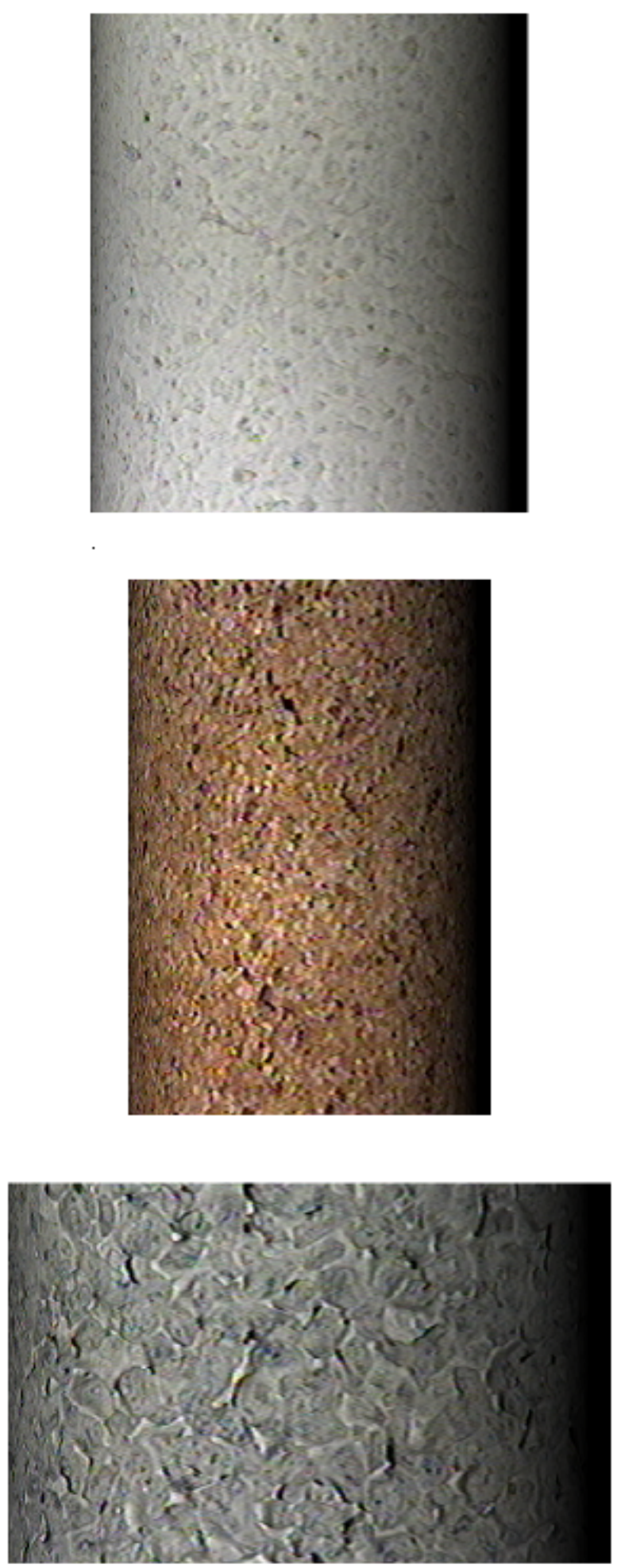
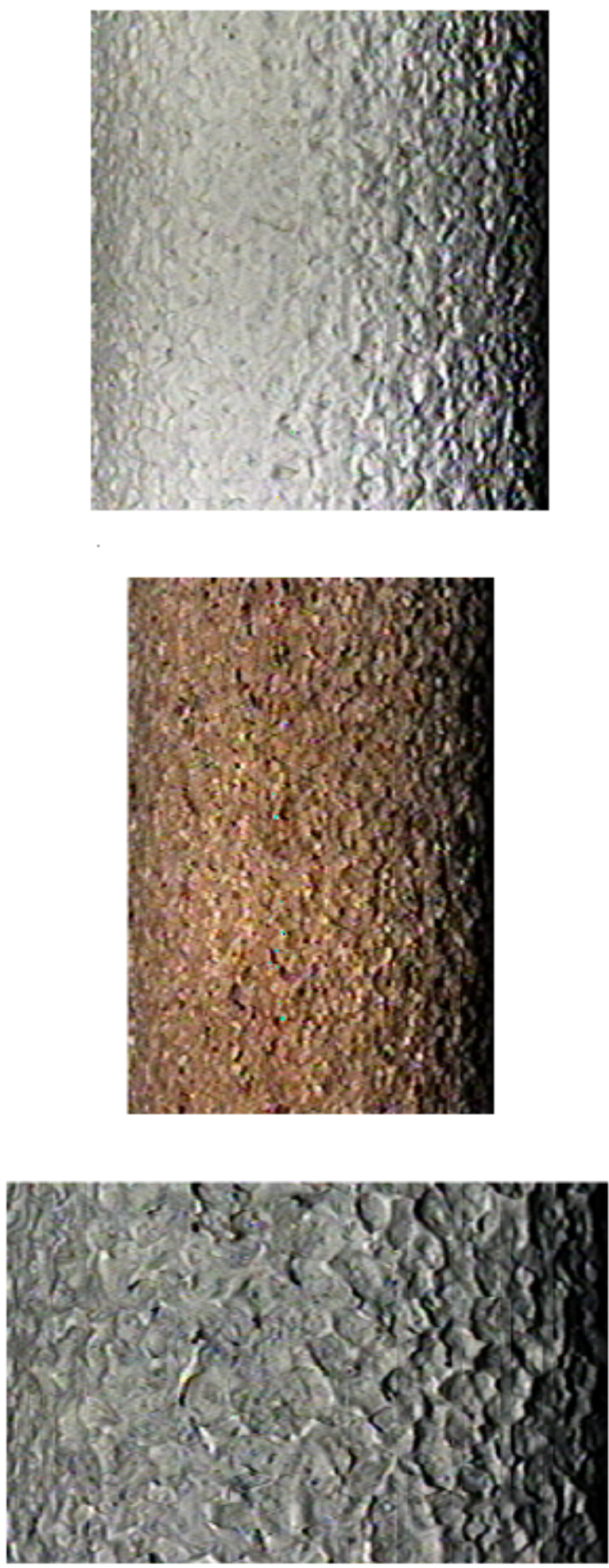

Figure 12: Cylinders rendered with 2D texture mapping (left) and 3D texture mapping (right). From top to bottom, the samples rendered are Sample 11 (plaster), Sample 8 (pebbles) and Sample 45 (concrete). 

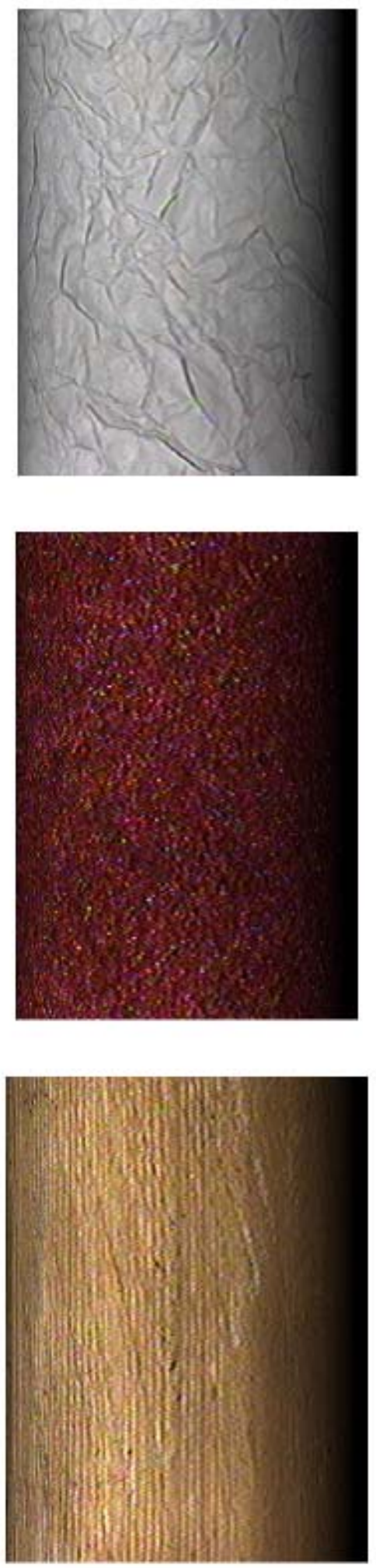
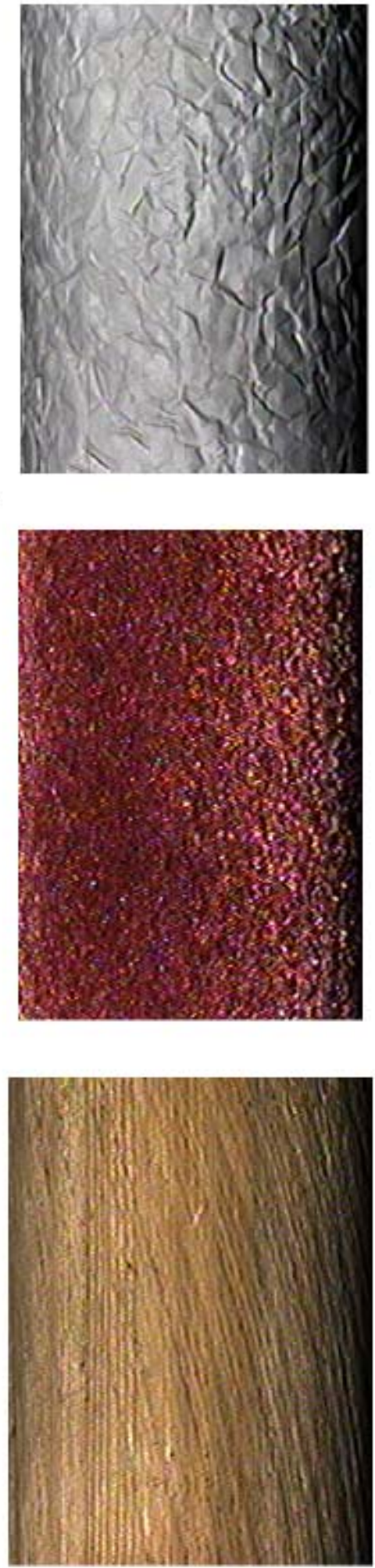

Figure 13: Cylinders rendered with 2D texture mapping (left) and 3D texture mapping (right). From top to bottom, the samples rendered are Sample 28 (crumpled paper), Sample 19 (rug) and Sample 56 (wood). 
Clearly 2D texture mapping can not account for the variations in texture appearance due to local shading, foreshortening, shadowing, occlusions and interreflections. These complex photometric and geometric effects significantly change the appearance of the surface texture. By using several images for 3D texture mapping of the cylinder, we can incorporate these effects and substantially improve the image realism. Consider the renderings shown in Figure 12 and Figure 13. The surface roughness of the cylinders rendered with 3D texture mapping is readily apparent, while the cylinders rendered with 2D texture mapping appear rather smooth. In some cases, like the concrete cylinder rendered in Figure 12, the cylinder's roughness appears to be flattened out so that it appears as shallow indentations. Even a complex surface geometry like that of the plush rug shown in the center row of Figure 13 is well-rendered with this simple 3D texture mapping, while the corresponding 2D texture-mapped cylinder hardly resembles a rug. The surface of the wood cylinder depicted in the last row of Figure 13 has machined grooves that are more noticeable when the surface is tilted away from the observer. This effect can be observed in the wood cylinder rendered with 3D texture mapping. However, the 2D texture-mapped version of the wood cylinder shows these grooves disappearing as the view becomes oblique.

These rendered cylinders demonstrate the potential of 3D texture mapping, but there are many unresolved issues. For instance, interpolation must be done between measured BTF images. Also, seams become a problem when the sizes of the characteristic texture elements become large compared to the size of the patch being textured. The database presented here is a starting point for further exploration into this area.

\section{$5 \quad$ Fitting to BRDF Models}

A concise description is required for functional utility of the measurements. We employ the OrenNayar model [15] and the Koenderink et al. representation [10] to obtain parametric descriptions of the BRDF measurement database. The resulting database of parameters can be used directly and conveniently in a variety of algorithms where accurate, concise and analytical reflectance descriptions are needed. In vision, these applications include shape-from-shading and photometric stereo. In computer graphics, the reflectance parameters are useful for realistic rendering of natural surfaces. As with the measurement database, the complete database of reflectance parameters is also available electronically at http://www.cs.columbia.edu/CAVE/curet.

\subsection{Oren-Nayar Model}

The details of the derivation of the Oren-Nayar reflectance model can be found in [15]; the main results are summarized here. In the Oren-Nayar reflectance model, a surface patch imaged by each sensor detector is modeled as a collection of numerous long symmetric V-shaped cavities; each cavity has two planar Lambertian facets with opposing normals, facet normals are free to deviate from the mean surface normal, and all facets on the surface have the same albedo $\rho$. It is assumed that the $\mathrm{V}$-cavities are uniformly distributed in orientation $\phi_{a}$ (azimuth angle) on the surface plane, whereas facet tilt $\theta_{a}$ (polar angle) is normally distributed with zero mean and standard deviation $\sigma$, the latter serving as a macroscopic roughness parameter. The geometry of the $\mathrm{V}$-groove is used to capture effects of foreshortening, masking, shadowing and interreflections (up to two bounces) between adjacent facets. As an example of these effects, consider an obliquely illuminated V-groove. One facet will be brighter since its local surface normal is closer to the source direction. Likewise, for non-frontal views, the amount of foreshortening is different for each of the facets. When the brighter facet is foreshortened more than the darker facet, the overall brightness of the V-groove 
decreases. Similarly, masking of the brighter facet decreases average brightness.

The total brightness of a surface patch is expressed as the integral of facet brightness over all facet normals. The complexities of foreshortening, masking, shadowing and interreflection makes this integral difficult to evaluate. A solution is obtained by first deriving a basis function for each component of the integral and then finding coefficients for the bases through extensive numerical simulations.

Define the viewing direction with the azimuthal and polar angles $\phi_{r}$ and $\theta_{r}$, respectively, and similarly define the illumination direction with $\phi_{i}$ and $\theta_{i}$. The diffuse radiance of the surface $L_{d}$ is expressed as

$$
L_{d}=L_{1}+L_{2}
$$

where $L_{1}$ is the contribution of the primary reflectance and $L_{2}$ is the contribution of interreflections. The final approximations for these components are

$$
\begin{aligned}
L_{1}= & \frac{\rho}{\pi} E_{0} \cos \theta_{i}\left[C_{1}(\sigma)\right. \\
& +\cos \left(\phi_{r}-\phi_{i}\right) C_{2}\left(\alpha ; \beta ; \phi_{r}-\phi_{i} ; \sigma\right) \tan \beta \\
& \left.+\left(1-\left|\cos \left(\phi_{r}-\phi_{i}\right)\right|\right) C_{3}(\alpha ; \beta ; \sigma) \tan \left(\frac{\alpha+\beta}{2}\right)\right], \\
L_{2}= & \frac{0.17 \rho^{2} \sigma^{2}}{\pi\left(\sigma^{2}+0.13\right)} E_{0} \cos \theta_{i}\left[1-\cos \left(\phi_{r}-\phi_{i}\right)\left(\frac{2 \beta}{\pi}\right)^{2}\right],
\end{aligned}
$$

where $\rho$ is the albedo of the facets, $E_{0} \cos \theta_{i}$ is the irradiance, $\alpha=\max \left[\theta_{r}, \theta_{i}\right]$ and $\beta=\min \left[\theta_{r}, \theta_{i}\right]$. The coefficients of the model are

$$
\begin{gathered}
C_{1}=1-0.5 \frac{\sigma^{2}}{\sigma^{2}+0.33}, \\
C_{2}=\begin{array}{ll}
0.45 \frac{\sigma^{2}}{\sigma^{2}+0.09} \sin \alpha & \text { if } \cos \left(\phi_{r}-\phi_{i}\right) \geq 0 \\
0.45 \frac{\sigma^{2}}{\sigma^{2}+0.09}\left(\sin \alpha-\left(\frac{2 \beta}{\pi}\right)^{3}\right) & \text { otherwise }
\end{array}
\end{gathered}
$$

and

$$
C_{3}=0.125 \frac{\sigma^{2}}{\sigma^{2}+0.09}\left(\frac{4 \alpha \beta}{\pi^{2}}\right)^{2},
$$

where $\sigma$ is the standard deviation of facet slope and hence serves as the surface roughness parameter. Notice that interchanging the source and viewing direction does not change the the ratio of radiance $\left(L_{d}\right)$ to irradiance $\left(E_{0} \cos \theta_{i}\right)$, indicating that this model obeys Helmholtz's reciprocity condition. To account for specular reflection, the Torrance-Sparrow model[25] is incorporated so that the total radiance $L$ is given by

$$
L\left(\theta_{r}, \theta_{i}, \phi_{r}-\phi_{i} ; \rho, \sigma\right)=k_{d} L_{d}+k_{s} L_{s},
$$

where $L_{s}$ is determined by the Torrance-Sparrow model, $k_{d}$ and $k_{s}$ are the diffuse and specular components, and $k_{d}=1-k_{s}$. A key benefit of this model is that it has only three unknown parameters: $\rho, \sigma$ and $k_{d}$. 


\subsection{Koenderink et al. Representation}

In order to interpolate BRDF data sets, a series development in terms of an orthonormal basis can be used. Koenderink et al. [9] have shown how to construct such a complete, orthonormal basis on the Cartesian product of the hemisphere. Their set of polynomials, called surface scattering modes, is ordered with respect to angular resolution and automatically respects Helmholtz's reciprocity and (if desired) surface isotropy. The derivation uses the Zernike circle polynomials [2] that form a complete basis for functions on the interior of the unit disk. By applying an area true mapping of the hemisphere on the unit disk, the Zernike polynomials are transformed into a complete orthonormal system $K_{n}^{l}(\theta, \phi)$ on $\mathbf{H}^{2}$ normalized so that

$$
\int_{\mathbf{H}^{2}} K_{n}^{l *} K_{n}^{l} d \Omega=\delta_{n n^{\prime}} \delta_{l l^{\prime}}
$$

The permissible values of the integers $n$ and $l$ are $n \geq 0,|l| \leq n$, and $(n-l)$ even. The functions $K_{n}^{l}$ are each the product of a polar function $\Theta_{n}^{l}(\theta)$ and an azimuthal function $G^{l}(\phi)$ :

$$
K_{n}^{l}(\theta, \phi)=\Theta_{n}^{l}(\theta) G^{l}(\phi)
$$

with

$$
\Theta_{n}^{l}(\theta)=\sqrt{\frac{n+1}{2 \pi}} R_{n}^{l}\left(\sqrt{2} \sin \frac{\theta}{2}\right)
$$

and

$$
G^{l}(\phi)=\exp (i l \phi)
$$

Instead of the complex functions $G^{l}$, we use real trigonometric functions. Positive values of $l$ correspond to cosine terms, negative values to sine terms:

$$
G^{l}(\phi)= \begin{cases}\cos (l \phi) & \text { for } l \geq 0 \\ \sin (|l| \phi) & \text { for } l<0\end{cases}
$$

The functions $R_{n}^{l}$ are closely related to Jacobi's polynomials [2] and are given by

$$
R_{n}^{ \pm l}(\tau)=\sum_{s=0}^{(n-l) / 2}(-1)^{s} \frac{(n-s) ! \tau^{n-2 s}}{s !((n+l) / 2-s) !((n-l) / 2-s) !}
$$

Helmholtz reciprocity expresses the fact that, in the approximation of geometrical optics, the direction of light rays may be reversed. Therefore, the BRDF does not change when the entrance and exit beams are interchanged. This implies that the development of the BRDF $f\left(\theta_{i}, \phi_{i}, \theta_{r}, \phi_{r}\right)$ in terms of $K_{n}^{l}$ can be written as

$$
\begin{gathered}
f\left(\theta_{i}, \phi_{i}, \theta_{r}, \phi_{r}\right)=\sum_{n l n^{\prime} l^{\prime}} a_{n l n^{\prime} l^{\prime}} H_{n n^{\prime}}^{l l^{\prime}}\left(\theta_{i}, \phi_{i}, \theta_{r}, \phi_{r}\right) \\
=\sum_{n l n^{\prime} l^{\prime}} a_{n l n^{\prime} l^{\prime}}\left(K_{n}^{l}\left(\theta_{i}, \phi_{i}\right) K_{n^{\prime}}^{l^{\prime}}\left(\theta_{r}, \phi_{r}\right)+K_{n^{\prime}}^{l^{\prime}}\left(\theta_{i}, \phi_{i}\right) K_{n}^{l}\left(\theta_{r}, \phi_{r}\right)\right),
\end{gathered}
$$

where $n \geq 0,|l| \leq n,(n-l)$ even, $n^{\prime} \leq n,\left|l^{\prime}\right| \leq n^{\prime},\left(n^{\prime}-l^{\prime}\right)$ even, and if $n^{\prime}=n$ then $l^{\prime} \leq l$. Explicit expressions for $H_{n n^{\prime}}^{l l^{\prime}}$ for order 0 and 1 are

$$
H_{00}^{00}\left(\theta_{i}, \phi_{i}, \theta_{r}, \phi_{r}\right)=\frac{1}{\pi}
$$




$$
\begin{aligned}
H_{00}^{1-1}\left(\theta_{i}, \phi_{i}, \theta_{r}, \phi_{r}\right) & =\frac{-1}{\pi}\left(\sin \phi_{i} \sin \frac{\theta_{i}}{2}+\sin \phi_{r} \sin \frac{\theta_{r}}{2}\right) \\
H_{1-1}^{1-1}\left(\theta_{i}, \phi_{i}, \theta_{r}, \phi_{r}\right) & =\frac{4}{\pi} \sin \phi_{i} \sin \frac{\theta_{i}}{2} \sin \phi_{r} \sin \frac{\theta_{r}}{2} \\
H_{00}^{11}\left(\theta_{i}, \phi_{i}, \theta_{r}, \phi_{r}\right) & =\frac{1}{\pi}\left(\cos \phi_{i} \sin \frac{\theta_{i}}{2}+\cos \phi_{r} \sin \frac{\theta_{r}}{2}\right) \\
H_{1-1}^{11}\left(\theta_{i}, \phi_{i}, \theta_{r}, \phi_{r}\right) & =\frac{-2}{\pi} \sin \left(\phi_{i}+\phi_{r}\right) \sin \frac{\theta_{i}}{2} \sin \frac{\theta_{r}}{2} \\
H_{11}^{11}\left(\theta_{i}, \phi_{i}, \theta_{r}, \phi_{r}\right) & =\frac{4}{\pi} \cos \phi_{i} \sin \frac{\theta_{i}}{2} \cos \phi_{r} \sin \frac{\theta_{r}}{2} .
\end{aligned}
$$

If the surface under consideration is isotropic, the BRDF depends only on $\left|\phi_{i}-\phi_{r}\right|$. This means that in the expansion we need to keep only terms containing $\cos \left(\phi_{i}-\phi_{r}\right)$. This greatly reduces the number of basis functions. If we set $\Delta \phi=\left|\phi_{i}-\phi_{r}\right|$, we can write the development in this system as

$$
\begin{gathered}
f\left(\theta_{i}, \phi_{i}, \Delta \phi\right)=\sum_{n m l} a_{n m l} S_{n m}^{l}\left(\theta_{i}, \phi_{i}, \Delta \phi\right) \\
=\sum_{n m l} a_{n m l}\left(\Theta_{n}^{l}\left(\theta_{i}\right) \Theta_{m}^{l}\left(\theta_{r}\right)+\Theta_{m}^{l}\left(\theta_{i}\right) \Theta_{n}^{l}\left(\theta_{r}\right)\right) \cos (l \Delta \phi),
\end{gathered}
$$

where $n \geq 0,0 \leq m \leq n, 0 \leq l \leq m,(n-l)$ and $(n-m)$ even. Explicit expressions for $S_{n m}^{l}$ up to order 2 are

$$
\begin{aligned}
S_{00}^{0}\left(\theta_{i}, \theta_{r}, \Delta \phi\right) & =\frac{1}{\pi} \\
S_{11}^{1}\left(\theta_{i}, \theta_{r}, \Delta \phi\right) & =\frac{4}{\pi} \sin \frac{\theta_{i}}{2} \sin \frac{\theta_{r}}{2} \cos \Delta \phi \\
S_{20}^{0}\left(\theta_{i}, \theta_{r}, \Delta \phi\right) & =\frac{\sqrt{3}}{\pi}\left(1-\cos \theta_{i}-\cos \theta_{r}\right) \\
S_{22}^{0}\left(\theta_{i}, \theta_{r}, \Delta \phi\right) & =\frac{3}{\pi}\left(1-2 \cos \theta_{i}\right)\left(1-2 \cos \theta_{r}\right) \\
S_{22}^{2}\left(\theta_{i}, \theta_{r}, \Delta \phi\right) & =\frac{3}{\pi}\left(1-\cos \theta_{i}\right)\left(1-\cos \theta_{r}\right) \cos (2 \Delta \phi) .
\end{aligned}
$$

Note that for both systems, the 0-order term is the BRDF for a perfect Lambertian reflector. The development of the BRDF in Equation 12 can be used for anisotropic samples, while the development in Equation 13 is applicable to isotropic samples. In practice, the series to be fitted should be truncated so that the highest frequency terms are still sampled by the experimental data. The following table gives the number of basis functions (and hence coefficients) per order for the first 8 orders of both systems:

\begin{tabular}{l|ccccccccc} 
order $n$ & 0 & 1 & 2 & 3 & 4 & 5 & 6 & 7 & 8 \\
\hline \# of $H_{n n^{\prime}}^{l l^{\prime}}$ & 1 & 5 & 15 & 34 & 65 & 111 & 175 & 260 & 369 \\
\# of $S_{n m}^{l}$ & 1 & 1 & 3 & 3 & 6 & 6 & 10 & 10 & 15
\end{tabular}

\subsection{BRDF Fitting Results}

The 3 parameter Oren-Nayar model was fit to the 205 radiance measurements for each sample using the Levenberg-Marquardt method. The series used in the Koenderink et al. representation 
was truncated at order 2 and order 8, resulting in a 5 parameter and a 55 parameter representation, respectively. Linear estimation was used to fit the truncated series to the BRDF measurements.

Plots for the entire set of fitting results are too numerous to include here and are provided in Appendix D. The samples included here were chosen to be representative of the overall results. These samples are 11-plaster, 12-rough paper, 49-concrete, 25-quarry tile, 21-sponge, and 20-styrofoam. The measurements and modeling results are depicted in Figure 14. Row A of Figure 14 shows the raw measurement data plotted as a function of decreasing source angle to emphasize the nonmonotonic and non-Lambertian behavior of the reflectance. Rows B,C and D show the scatter-plots of the Oren-Nayar model fit, the order 2 Koenderink et al. fit and the order 8 Koenderink et al. fit, respectively. These scatter-plots show the 205 measurement values plotted as a function of the corresponding estimated value; a straight line indicates a perfect fit. Rows E,F and G show the residuals from the Oren-Nayar model fit, the order 2 Koenderink et al. fit and the order 8 Koenderink et al. fit, respectively. The residuals are plotted as a function of increasing viewing angle to show the concentration of errors at oblique views for some of the samples. All measurements are shown in radiance with units of watts per steridian per square-meter.

For the Oren-Nayar model fits, the best performance is with diffuse samples, like plaster and concrete. Samples like quarry tile and styrofoam, which show sharp peaks in the radiance plots of Row A, have more fitting errors. For each of the fits shown, the average error is less than $10 \%$ of the peak reflectance value. For many applications this amount of error may be acceptable given the conciseness of this 3 parameter model.

It is useful to compare the performance of the order 2 Koenderink et al. decomposition and the Oren-Nayar model since these representations have approximately the same number of parameters. The performance is better for most cases using the Oren-Nayar model. The low orders of the Koenderink et al. model are best used for Lambertian-type reflectance (with order 0 identical to Lambertian reflectance). As a result, samples like the sponge and styrofoam are not wellrepresented.

The 55 parameter Koenderink et al. decomposition accurately represents each of the reflectance plots as shown by the near straight line scatter-plots for each sample in Row D. In fact, all of the samples in the database are represented accurately with this decomposition. Note that the behavior of the fit between measured points is not indicated by these plots. The possible presence of oscillations associated with high order fits needs further investigation.

\section{IMPLICATIONS FOR COMPUTER VISION}

Our BRDF measurement database provides a thorough investigation of the reflectance properties of real-world rough surfaces. This database fills a long-standing need for a benchmark to test and compare BRDF models as we have done here for the Oren-Nayar model and the Koenderink et al. decomposition. Such a comparison is useful in choosing a representation that has the right balance of accuracy and compactness for the application. In circumstances which require the use of simpler and less accurate reflectance models, e.g. the Lambertian model, our database is useful for gauging expected errors.

Our BRDF parameter database, obtained by fitting the measurements to the Oren-Nayar model and the Koenderink et al. decomposition, can be used in place of the popular Lambertian reflectance model in such algorithms as shape-from-shading [7] and photometric stereo [30]. Since these algorithms rely on a reflectance model to ascertain shape, inaccuracies of the Lambertian model can significantly affect their performance.

Since the parameter database covers two BRDF representations, a choice can be made to bal- 


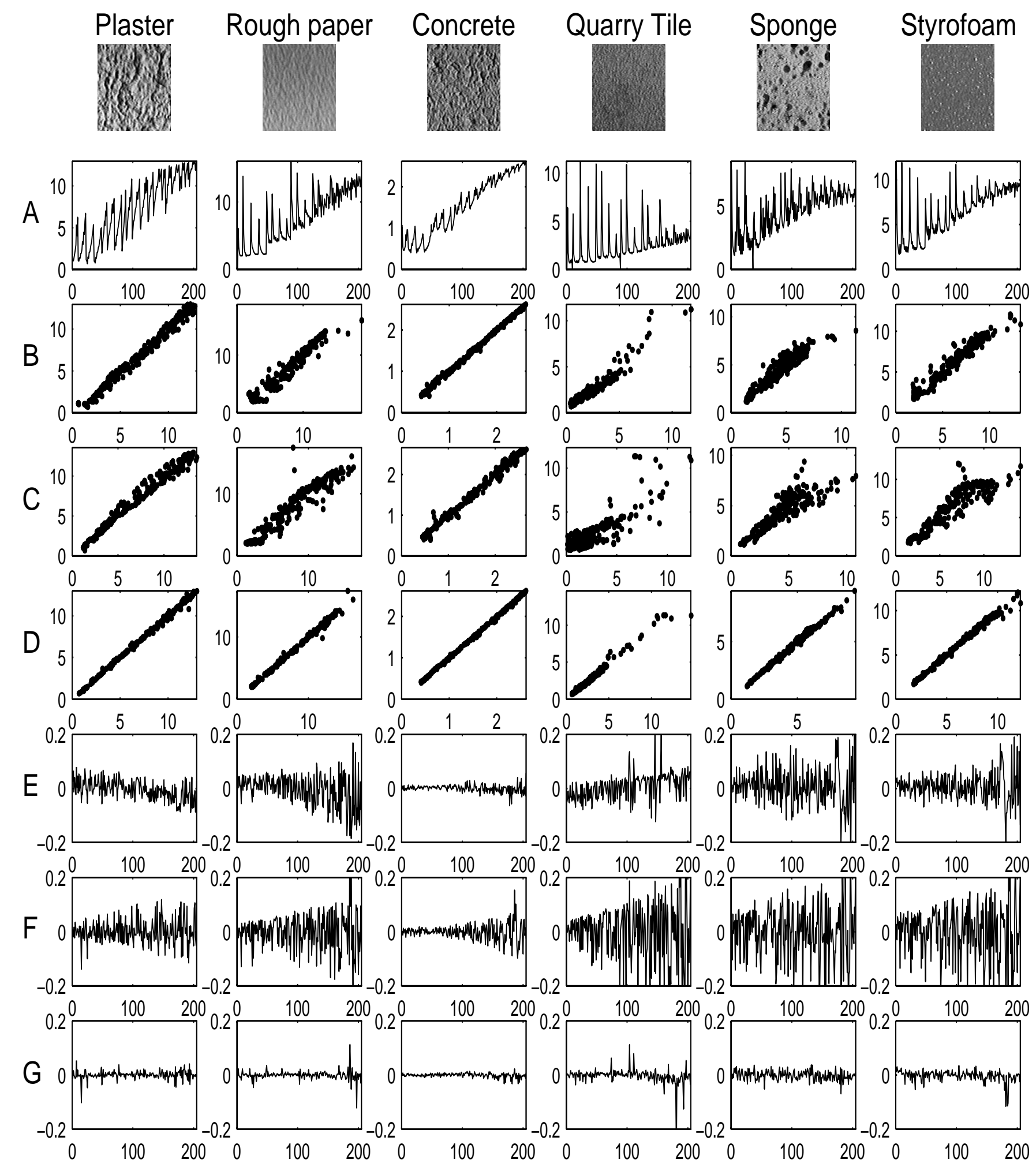

Figure 14: BRDF measurements and model-fitting results. Row A shows the raw measurement data plotted as a function of decreasing source angle to emphasize the non-Lambertian behavior of each sample. Rows B,C and D show the scatter-plots of the Oren-Nayar model fit ( 3 parameters), the order 2 Koenderink et al. fit ( 5 parameters) and the order 8 Koenderink et al. fit (55 parameters), respectively. Rows E,F and $\mathrm{G}$ show the residuals from the Oren-Nayar model fit, the order 2 Koenderink et al. fit and the order 8 Koenderink et al. fit, respectively. The residuals are plotted as a function of increasing viewing angle to show the concentration of errors at oblique views for some of the samples. The residuals shown are normalized by maximum measured radiance for that sample. 
ance accuracy and conciseness. For isotropic surfaces the 3-parameter Oren-Nayar model can be employed. For isotropic and anisotropic surfaces, when a richer description can be afforded, the 55 parameter Koenderink et al. model can be used. For the 61 surfaces we have investigated, the parameters for both models are readily available.

Our BTF database is the first comprehensive investigation of texture appearance as a function of viewing and illumination direction. As illustrated in Figure 10 and Figure 11, surface roughness causes notable effects on the BTF which are not considered by current texture algorithms. Present algorithms for shape-from-texture [16],[23],[11], texture segmentation [31],[11] and texture recognition [20] are only suitable for 2D textures, i.e. planar texture due to albedo variation. Texture analysis of real-world rough surfaces remains an important unsolved problem. The database illustrates the need for 3D texture algorithms and serves as a starting point for their exploration.

\section{Implications for Computer Graphics}

Our BTF measurement database is the first comprehensive investigation of texture appearance as a function of viewing and illumination directions. As illustrated in Figure 12 and Figure 13, changes of view and illumination cause notable effects on texture appearance which are not considered by current texture rendering algorithms. When the surface is rough, standard texture rendering tends to be too flat and unrealistic. Even if the rendering from a single view is suitably realistic, the realism is lost when the view or illumination changes. The database illustrates the need for 3D texture rendering algorithms and serves as a starting point for their exploration.

Our BRDF parameter database, obtained by fitting the measurements to the Oren-Nayar model and the Koenderink et al. decomposition, can be used directly in image rendering instead of popular shading models like [6],[3] for photorealistic rendering of real-world surfaces. Since the parameter database covers two BRDF representations, a choice can be made to balance accuracy and concise-

ness. For isotropic surfaces the 3-parameter Oren-Nayar model can be employed. For isotropic and anisotropic surfaces, when a richer description can be afforded, the 55 parameter Koenderink et al. model can be used. For the 61 surfaces we have investigated, the parameters for both models are readily available.

Our BTF measurement database, BRDF measurement database and BRDF model parameter database together represent an extensive investigation of the appearance of real-world surfaces. Each of these databases has important implications for computer vision and computer graphics and each is available at $h t t p: / / w w w . c s . c o l u m b i a . e d u / C A V E /$ curet.

\section{ACKNOWLEDGEMENTS}

The research is sponsored in part by the National Science Foundation, DARPA/ONR under the MURI Grant No. N00014-95-1-0601 and by REALISE of the European Commision, 


\section{APPENDIX}

\section{A IMAGING PARAMETERS FOR MEASUREMENTS}

To achieve the varied imaging conditions required for BRDF measurements the camera is moved to 7 different positions illustrated in Figure 5(b) and for each camera position the sample is reoriented so that the sample normal is directed toward each of the 64 vertices shown in Figure 5(a). Tables 1-7 quantify the resulting illumination and viewing direction by listing: sample orientation label (1-64), sample normal in world coordinates $n_{x}^{w}, n_{y}^{w}$ and $n_{z}^{w}$, polar viewing angle $\theta_{r}$, azimuthal viewing angle $\phi_{r}$, polar illumination angle $\theta_{i}$ and azimuthal illumination angle $\phi_{i}$. The viewing and illumination angles $\theta_{r} \phi_{r}, \theta_{i}$ and $\phi_{i}$ are expressed in the sample coordinate frame.

\section{B IMAGE SEGMENTATION}

The image of each texture sample contains unnecessary background information depicting the sample base and the robot gripper. The purpose of image segmentation is to define the boundary of useful information so that subsequent applications can readily ignore background data. The method of segmenting the images is a combination of manual corner selection and automatic corner estimation using the known sample positions. Specifically, the boundaries are manually chosen for a subset of the image set for a given sample. Using these image-based corner locations and the known position of the sample and camera, the corner locations in a sample-base coordinate frame are estimated using linear regression. These corner points are then transformed to predict the corner locations in the remaining images of the set. For a conservative estimate of the valid data region, the area of the predicted region is slightly reduced. The resulting boundary is stored in a *. cnr file in the format of 8 consecutive integers representing the $(x, y)$ corner coordinates of the polygonal boundary.

\section{B.1 COORDINATE FRAME TRANSFORMATIONS}

Figure 3 depicts the measurement equipment comprised of a robot, lamp and camera. Three coordinate frames are illustrated in this figure: the world coordinate frame $\mathbf{x}_{\mathbf{w}}-\mathbf{y}_{\mathbf{w}}-\mathbf{z}_{\mathbf{w}}$, the sample coordinate frame $\mathbf{x}_{\mathbf{s}}-\mathbf{y}_{\mathbf{s}}-\mathbf{z}_{\mathbf{s}}$, and the image coordinate frame $\mathbf{x}_{\mathbf{i}}-\mathbf{y}_{\mathbf{i}}-\mathbf{z}_{\mathbf{i}}$. A halogen lamp with a Fresnel lens was positioned to illuminate the sample along $\mathbf{x}_{\mathbf{w}}$. The camera is shown in one of its seven positions, i.e. with the viewing direction along $\mathbf{y}_{\mathbf{w}}$. The world coordinate frame is fixed; however, the sample coordinate frame is attached to the sample and changes with the sample position. Likewise, the image coordinate frame is defined relative to the camera and changes with the camera position.

Each corner of the polygonal boundary of valid data can be represented by a $2 \times 1$ vector for the $x-y$ coordinates. Let the vectors $\mathbf{a}_{\mathbf{i}}, \mathbf{b}_{\mathbf{i}}, \mathbf{c}_{\mathbf{i}}, \mathbf{d}_{\mathbf{i}}$ represent the four corners of the boundary $\left(\mathbf{a}_{\mathbf{i}}\right.$ is upper left corner and the remaining corners are ordered clockwise). Let $\mathbf{R}_{\mathbf{w}}^{\mathrm{i}}$ be the rotation matrix which transforms points from world to image coordinates. Specifically, for the camera in position 4 , i.e. for the viewing direction along $\mathbf{y}_{\mathbf{w}}$,

$$
\mathbf{R}_{\mathrm{w}}^{\mathbf{i}}=\left[\begin{array}{ccc}
1 & 0 & 0 \\
0 & 0 & -1 \\
0 & 1 & 0
\end{array}\right]
$$




\begin{tabular}{|c|c|c|c|c|c|c|c|c|}
\hline Image No. & Vertex & $n_{x}^{w}$ & $n_{y}^{w}$ & $n_{z}^{w}$ & $\theta_{r}$ & $\phi_{r}$ & $\theta_{i}$ & $\phi_{i}$ \\
\hline 1 & 2 & 0.195090 & 0.000000 & 0.980785 & 1.389566 & -1.970402 & 1.374447 & -1.570796 \\
\hline 2 & 3 & 0.195090 & 0.980785 & 0.000000 & 0.981748 & 0.000000 & 1.374447 & 0.000000 \\
\hline 3 & 5 & 0.198757 & -0.198757 & 0.959683 & 1.463021 & -2.763231 & 1.370707 & -2.376765 \\
\hline 4 & 6 & 0.198757 & 0.198757 & 0.959683 & 1.308097 & -1.163336 & 1.370707 & -0.764828 \\
\hline 5 & 7 & 0.198757 & 0.959683 & 0.198757 & 0.987375 & -0.134267 & 1.370707 & -0.041141 \\
\hline 6 & 10 & 0.208847 & 0.404615 & 0.890320 & 1.215585 & -0.811489 & 1.360401 & -0.430767 \\
\hline 7 & 11 & 0.208847 & 0.890320 & 0.404615 & 1.007874 & -0.282959 & 1.360401 & -0.094629 \\
\hline 8 & 14 & 0.211325 & 0.577350 & 0.788675 & 1.141555 & -0.627520 & 1.357866 & -0.281035 \\
\hline 9 & 15 & 0.211325 & 0.788675 & 0.577350 & 1.050598 & -0.417031 & 1.357866 & -0.153484 \\
\hline 10 & 17 & 0.382683 & 0.000000 & 0.923880 & 1.209430 & -1.992270 & 1.178098 & -1.570796 \\
\hline 11 & 18 & 0.382683 & 0.923880 & 0.000000 & 0.785399 & 0.000000 & 1.178098 & 0.000000 \\
\hline 12 & 20 & 0.404615 & -0.208847 & 0.890320 & 1.272499 & -2.496589 & 1.154239 & -2.096192 \\
\hline 13 & 21 & 0.404615 & 0.208847 & 0.890320 & 1.099841 & -1.476736 & 1.154239 & -1.045400 \\
\hline 14 & 22 & 0.404615 & 0.890320 & 0.208847 & 0.774850 & -0.219877 & 1.154239 & -0.094629 \\
\hline 15 & 23 & 0.408248 & -0.408248 & 0.816497 & 1.348016 & -2.815465 & 1.150262 & -2.456873 \\
\hline 16 & 24 & 0.408248 & 0.408248 & 0.816497 & 1.008179 & -1.101321 & 1.150262 & -0.684719 \\
\hline 17 & 26 & 0.408248 & 0.816497 & 0.408248 & 0.809816 & -0.439945 & 1.150262 & -0.201358 \\
\hline 18 & 27 & 0.426401 & -0.639603 & 0.639603 & 1.421060 & -3.015733 & 1.130287 & -2.738536 \\
\hline 19 & 28 & 0.426401 & 0.639603 & 0.639603 & 0.877978 & -0.762419 & 1.130287 & -0.403056 \\
\hline 20 & 29 & 0.555570 & -0.831470 & 0.000000 & 1.374447 & 3.141593 & 0.981748 & 3.141593 \\
\hline 21 & 30 & 0.555570 & 0.000000 & 0.831470 & 1.031794 & -2.032979 & 0.981748 & -1.570796 \\
\hline 22 & 31 & 0.555570 & 0.831470 & 0.000000 & 0.589049 & 0.000000 & 0.981748 & 0.000000 \\
\hline 23 & 32 & 0.577350 & -0.788675 & 0.211325 & 1.337086 & -3.090099 & 0.955317 & -2.988109 \\
\hline 24 & 33 & 0.577350 & -0.211325 & 0.788675 & 1.101194 & -2.432726 & 0.955317 & -2.005315 \\
\hline 25 & 34 & 0.577350 & 0.211325 & 0.788675 & 0.909333 & -1.623802 & 0.955317 & -1.136277 \\
\hline 26 & 35 & 0.577350 & 0.788675 & 0.211325 & 0.582273 & -0.334581 & 0.955317 & -0.153484 \\
\hline 27 & 36 & 0.639603 & -0.426401 & 0.639603 & 1.128806 & -2.736898 & 0.876815 & -2.376909 \\
\hline 28 & 37 & 0.639603 & 0.426401 & 0.639603 & 0.716526 & -1.270836 & 0.876815 & -0.764684 \\
\hline 29 & 38 & 0.639603 & -0.639603 & 0.426401 & 1.217332 & -2.966775 & 0.876815 & -2.738536 \\
\hline 30 & 39 & 0.639603 & 0.639603 & 0.426401 & 0.581425 & -0.799895 & 0.876815 & -0.403056 \\
\hline 31 & 40 & 0.707107 & -0.707107 & 0.000000 & 1.178097 & 3.141593 & 0.785398 & 3.141593 \\
\hline 32 & 41 & 0.707107 & 0.000000 & 0.707107 & 0.858886 & -2.100699 & 0.785398 & -1.570796 \\
\hline 33 & 42 & 0.707107 & 0.707107 & 0.000000 & 0.392699 & 0.000000 & 0.785398 & 0.000000 \\
\hline 34 & 43 & 0.788675 & -0.577350 & 0.211325 & 1.038285 & -3.013835 & 0.662145 & -2.860557 \\
\hline 35 & 44 & 0.788675 & -0.211325 & 0.577350 & 0.866142 & -2.496546 & 0.662145 & -2.005315 \\
\hline 36 & 45 & 0.788675 & 0.211325 & 0.577350 & 0.627477 & -1.794991 & 0.662145 & -1.136277 \\
\hline 37 & 46 & 0.788675 & 0.577350 & 0.211325 & 0.318893 & -0.713993 & 0.662145 & -0.281035 \\
\hline 38 & 47 & 0.816497 & -0.408248 & 0.408248 & 0.929649 & -2.801295 & 0.615479 & -2.456873 \\
\hline 39 & 48 & 0.816497 & 0.408248 & 0.408248 & 0.426124 & -1.398445 & 0.615479 & -0.684719 \\
\hline 40 & 49 & 0.831470 & -0.555570 & 0.000000 & 0.981747 & 3.141593 & 0.589048 & 3.141593 \\
\hline 41 & 50 & 0.831470 & 0.000000 & 0.555570 & 0.694806 & -2.211453 & 0.589048 & -1.570796 \\
\hline 42 & 51 & 0.831470 & 0.555570 & 0.000000 & 0.196349 & 0.000000 & 0.589048 & 0.000000 \\
\hline 43 & 52 & 0.890320 & -0.404615 & 0.208847 & 0.839669 & -2.948852 & 0.472749 & -2.710826 \\
\hline 44 & 53 & 0.890320 & -0.208847 & 0.404615 & 0.733813 & -2.628785 & 0.472749 & -2.096192 \\
\hline 45 & 54 & 0.890320 & 0.208847 & 0.404615 & 0.445325 & -1.955310 & 0.472749 & -1.045400 \\
\hline 46 & 55 & 0.890320 & 0.404615 & 0.208847 & 0.213063 & -1.410017 & 0.472749 & -0.430767 \\
\hline 47 & 56 & 0.923880 & -0.382683 & 0.000000 & 0.785398 & 3.141593 & 0.392699 & 3.141593 \\
\hline 48 & 57 & 0.923880 & 0.000000 & 0.382683 & 0.548028 & -2.395741 & 0.392699 & -1.570796 \\
\hline 49 & 58 & 0.923880 & 0.382683 & 0.000000 & 0.000001 & 3.141593 & 0.392699 & 0.000000 \\
\hline 50 & 59 & 0.959683 & -0.198757 & 0.198757 & 0.625671 & -2.857075 & 0.284924 & -2.376765 \\
\hline 51 & 60 & 0.959683 & 0.198757 & 0.198757 & 0.274014 & -2.335623 & 0.284924 & -0.764828 \\
\hline 52 & 61 & 0.980785 & -0.195090 & 0.000000 & 0.589048 & 3.141593 & 0.196349 & 3.141593 \\
\hline 53 & 62 & 0.980785 & 0.000000 & 0.195090 & 0.436759 & -2.701422 & 0.196349 & -1.570796 \\
\hline 54 & 63 & 0.980785 & 0.195090 & 0.000000 & 0.196350 & 3.141593 & 0.196349 & 0.000000 \\
\hline 55 & 64 & 1.000000 & 0.000000 & 0.000000 & 0.392699 & 3.141593 & 0.000000 & 0.000000 \\
\hline
\end{tabular}

Table 1: Sample normal and corresponding viewing and illumination angles for Camera Position 1. 


\begin{tabular}{|c|c|c|c|c|c|c|c|c|}
\hline Image No. & Vertex & $n_{x}^{w}$ & $n_{y}^{w}$ & $n_{z}^{w}$ & $\theta_{r}$ & $\phi_{r}$ & $\overline{\theta_{i}}$ & $\overline{\phi_{i}}$ \\
\hline 56 & 2 & 0.195090 & 0.000000 & 0.980785 & 1.432405 & -2.365895 & 1.374447 & -1.570796 \\
\hline 57 & 3 & 0.195090 & 0.980785 & 0.000000 & 0.589049 & 0.000000 & 1.374447 & 0.000000 \\
\hline 58 & 6 & 0.198757 & 0.198757 & 0.959683 & 1.285872 & -1.570796 & 1.370707 & -0.764828 \\
\hline 59 & 7 & 0.198757 & 0.959683 & 0.198757 & 0.610885 & -0.293831 & 1.370707 & -0.041141 \\
\hline 60 & 10 & 0.208847 & 0.404615 & 0.890320 & 1.122109 & -1.226629 & 1.360401 & -0.430767 \\
\hline 61 & 11 & 0.208847 & 0.890320 & 0.404615 & 0.680547 & -0.578211 & 1.360401 & -0.094629 \\
\hline 62 & 14 & 0.211325 & 0.577350 & 0.788675 & 0.979211 & -1.038908 & 1.357866 & -0.281035 \\
\hline 63 & 15 & 0.211325 & 0.788675 & 0.577350 & 0.785398 & -0.785398 & 1.357866 & -0.153484 \\
\hline 64 & 17 & 0.382683 & 0.000000 & 0.923880 & 1.296783 & -2.395740 & 1.178098 & -1.570796 \\
\hline 65 & 18 & 0.382683 & 0.923880 & 0.000000 & 0.392700 & 0.000000 & 1.178098 & 0.000000 \\
\hline 66 & 20 & 0.404615 & -0.208847 & 0.890320 & 1.431921 & -2.864769 & 1.154239 & -2.096192 \\
\hline 67 & 21 & 0.404615 & 0.208847 & 0.890320 & 1.122109 & -1.914964 & 1.154239 & -1.045400 \\
\hline 68 & 22 & 0.404615 & 0.890320 & 0.208847 & 0.413656 & -0.508053 & 1.154239 & -0.094629 \\
\hline 69 & 24 & 0.408248 & 0.408248 & 0.816497 & 0.955317 & -1.570796 & 1.150262 & -0.684719 \\
\hline 70 & 26 & 0.408248 & 0.816497 & 0.408248 & 0.523599 & -0.886076 & 1.150262 & -0.201358 \\
\hline 71 & 28 & 0.426401 & 0.639603 & 0.639603 & 0.717004 & -1.267733 & 1.130287 & -0.403056 \\
\hline 72 & 30 & 0.555570 & 0.000000 & 0.831470 & 1.167071 & -2.447955 & 0.981748 & -1.570796 \\
\hline 73 & 31 & 0.555570 & 0.831470 & 0.000000 & 0.196350 & 0.000000 & 0.981748 & 0.000000 \\
\hline 74 & 33 & 0.577350 & -0.211325 & 0.788675 & 1.308997 & -2.790714 & 0.955317 & -2.005315 \\
\hline 75 & 34 & 0.577350 & 0.211325 & 0.788675 & 0.979211 & -2.102685 & 0.955317 & -1.136277 \\
\hline 76 & 35 & 0.577350 & 0.788675 & 0.211325 & 0.261800 & -0.938882 & 0.955317 & -0.153484 \\
\hline 77 & 36 & 0.639603 & -0.426401 & 0.639603 & 1.419463 & -3.014362 & 0.876815 & -2.376909 \\
\hline 78 & 37 & 0.639603 & 0.426401 & 0.639603 & 0.717004 & -1.873860 & 0.876815 & -0.764684 \\
\hline 79 & 39 & 0.639603 & 0.639603 & 0.426401 & 0.440510 & -1.570796 & 0.876815 & -0.403056 \\
\hline 80 & 41 & 0.707107 & 0.000000 & 0.707107 & 1.047198 & -2.526113 & 0.785398 & -1.570796 \\
\hline 81 & 42 & 0.707107 & 0.707107 & 0.000000 & 0.000000 & 0.000000 & 0.785398 & 0.000000 \\
\hline 82 & 43 & 0.788675 & -0.577350 & 0.211325 & 1.420805 & -3.108912 & 0.662145 & -2.860557 \\
\hline 83 & 44 & 0.788675 & -0.211325 & 0.577350 & 1.150262 & -2.819842 & 0.662145 & -2.005315 \\
\hline 84 & 45 & 0.788675 & 0.211325 & 0.577350 & 0.785398 & -2.356194 & 0.662145 & -1.136277 \\
\hline 85 & 46 & 0.788675 & 0.577350 & 0.211325 & 0.261800 & -2.202711 & 0.662145 & -0.281035 \\
\hline 86 & 47 & 0.816497 & -0.408248 & 0.408248 & 1.277953 & -3.006341 & 0.615479 & -2.456873 \\
\hline 87 & 48 & 0.816497 & 0.408248 & 0.408248 & 0.523599 & -2.255517 & 0.615479 & -0.684719 \\
\hline 88 & 49 & 0.831470 & -0.555570 & 0.000000 & 1.374446 & 3.141593 & 0.589048 & 3.141593 \\
\hline 89 & 50 & 0.831470 & 0.000000 & 0.555570 & 0.942289 & -2.634483 & 0.589048 & -1.570796 \\
\hline 90 & 51 & 0.831470 & 0.555570 & 0.000000 & 0.196350 & 3.141593 & 0.589048 & 0.000000 \\
\hline 91 & 52 & 0.890320 & -0.404615 & 0.208847 & 1.220213 & -3.063418 & 0.472749 & -2.710826 \\
\hline 92 & 53 & 0.890320 & -0.208847 & 0.404615 & 1.068004 & -2.895807 & 0.472749 & -2.096192 \\
\hline 93 & 54 & 0.890320 & 0.208847 & 0.404615 & 0.680547 & -2.563381 & 0.472749 & -1.045400 \\
\hline 94 & 55 & 0.890320 & 0.404615 & 0.208847 & 0.413656 & -2.633539 & 0.472749 & -0.430767 \\
\hline 95 & 56 & 0.923880 & -0.382683 & 0.000000 & 1.178097 & 3.141593 & 0.392699 & 3.141593 \\
\hline 96 & 57 & 0.923880 & 0.000000 & 0.382683 & 0.858886 & -2.776103 & 0.392699 & -1.570796 \\
\hline 97 & 58 & 0.923880 & 0.382683 & 0.000000 & 0.392700 & 3.141593 & 0.392699 & 0.000000 \\
\hline 98 & 59 & 0.959683 & -0.198757 & 0.198757 & 1.002667 & -3.011773 & 0.284924 & -2.376765 \\
\hline 99 & 60 & 0.959683 & 0.198757 & 0.198757 & 0.610885 & -2.847762 & 0.284924 & -0.764828 \\
\hline 100 & 61 & 0.980785 & -0.195090 & 0.000000 & 0.981747 & 3.141593 & 0.196349 & 3.141593 \\
\hline 101 & 62 & 0.980785 & 0.000000 & 0.195090 & 0.804433 & -2.948923 & 0.196349 & -1.570796 \\
\hline 102 & 63 & 0.980785 & 0.195090 & 0.000000 & 0.589049 & 3.141593 & 0.196349 & 0.000000 \\
\hline 103 & 64 & 1.000000 & 0.000000 & 0.000000 & 0.785398 & 3.141593 & 0.000000 & 0.000000 \\
\hline
\end{tabular}

Table 2: Sample normal and corresponding viewing and illumination angle for Camera Position 2 


\begin{tabular}{|c|c|c|c|c|c|c|c|c|}
\hline Image No. & Vertex & $n_{x}^{w}$ & $n_{y}^{w}$ & $n_{z}^{w}$ & $\theta_{r}$ & $\phi_{r}$ & $\theta_{i}$ & $\phi_{i}$ \\
\hline 104 & 3 & 0.195090 & 0.980785 & 0.000000 & 0.196350 & 0.000000 & 1.374447 & 0.000000 \\
\hline 105 & 6 & 0.198757 & 0.198757 & 0.959683 & 1.308097 & -1.978257 & 1.370707 & -0.764828 \\
\hline 106 & 7 & 0.198757 & 0.959683 & 0.198757 & 0.274014 & -0.805969 & 1.370707 & -0.041141 \\
\hline 107 & 10 & 0.208847 & 0.404615 & 0.890320 & 1.099841 & -1.664856 & 1.360401 & -0.430767 \\
\hline 108 & 11 & 0.208847 & 0.890320 & 0.404615 & 0.445325 & -1.186283 & 1.360401 & -0.094629 \\
\hline 109 & 14 & 0.211325 & 0.577350 & 0.788675 & 0.909333 & -1.517790 & 1.357866 & -0.281035 \\
\hline 110 & 15 & 0.211325 & 0.788675 & 0.577350 & 0.627477 & -1.346601 & 1.357866 & -0.153484 \\
\hline 111 & 17 & 0.382683 & 0.000000 & 0.923880 & 1.423821 & -2.776103 & 1.178098 & -1.570796 \\
\hline 112 & 18 & 0.382683 & 0.923880 & 0.000000 & 0.000001 & 0.000000 & 1.178098 & 0.000000 \\
\hline 113 & 21 & 0.404615 & 0.208847 & 0.890320 & 1.215585 & -2.330104 & 1.154239 & -1.045400 \\
\hline 114 & 22 & 0.404615 & 0.890320 & 0.208847 & 0.213063 & -1.731576 & 1.154239 & -0.094629 \\
\hline 115 & 24 & 0.408248 & 0.408248 & 0.816497 & 1.008179 & -2.040271 & 1.150262 & -0.684719 \\
\hline 116 & 26 & 0.408248 & 0.816497 & 0.408248 & 0.426124 & -1.743148 & 1.150262 & -0.201358 \\
\hline 117 & 28 & 0.426401 & 0.639603 & 0.639603 & 0.716526 & -1.870756 & 1.130287 & -0.403056 \\
\hline 118 & 30 & 0.555570 & 0.000000 & 0.831470 & 1.356554 & -2.809910 & 0.981748 & -1.570796 \\
\hline 119 & 31 & 0.555570 & 0.831470 & 0.000000 & 0.196349 & 3.141593 & 0.981748 & 0.000000 \\
\hline 120 & 34 & 0.577350 & 0.211325 & 0.788675 & 1.141555 & -2.514072 & 0.955317 & -1.136277 \\
\hline 121 & 35 & 0.577350 & 0.788675 & 0.211325 & 0.318893 & -2.427600 & 0.955317 & -0.153484 \\
\hline 122 & 37 & 0.639603 & 0.426401 & 0.639603 & 0.877978 & -2.379174 & 0.876815 & -0.764684 \\
\hline 123 & 39 & 0.639603 & 0.639603 & 0.426401 & 0.581425 & -2.341698 & 0.876815 & -0.403056 \\
\hline 124 & 41 & 0.707107 & 0.000000 & 0.707107 & 1.296782 & -2.856669 & 0.785398 & -1.570796 \\
\hline 125 & 42 & 0.707107 & 0.707107 & 0.000000 & 0.392699 & 3.141593 & 0.785398 & 0.000000 \\
\hline 126 & 44 & 0.788675 & -0.211325 & 0.577350 & 1.464020 & -3.065729 & 0.662145 & -2.005315 \\
\hline 127 & 45 & 0.788675 & 0.211325 & 0.577350 & 1.050598 & -2.724561 & 0.662145 & -1.136277 \\
\hline 128 & 46 & 0.788675 & 0.577350 & 0.211325 & 0.582273 & -2.807012 & 0.662145 & -0.281035 \\
\hline 129 & 48 & 0.816497 & 0.408248 & 0.408248 & 0.809816 & -2.701648 & 0.615479 & -0.684719 \\
\hline 130 & 50 & 0.831470 & 0.000000 & 0.555570 & 1.246977 & -2.915406 & 0.589048 & -1.570796 \\
\hline 131 & 51 & 0.831470 & 0.555570 & 0.000000 & 0.589049 & 3.141593 & 0.589048 & 0.000000 \\
\hline 132 & 53 & 0.890320 & -0.208847 & 0.404615 & 1.422492 & -3.075442 & 0.472749 & -2.096192 \\
\hline 133 & 54 & 0.890320 & 0.208847 & 0.404615 & 1.007874 & -2.858633 & 0.472749 & -1.045400 \\
\hline 134 & 55 & 0.890320 & 0.404615 & 0.208847 & 0.774850 & -2.921715 & 0.472749 & -0.430767 \\
\hline 135 & 57 & 0.923880 & 0.000000 & 0.382683 & 1.209429 & -2.984388 & 0.392699 & -1.570796 \\
\hline 136 & 58 & 0.923880 & 0.382683 & 0.000000 & 0.785399 & 3.141593 & 0.392699 & 0.000000 \\
\hline 137 & 59 & 0.959683 & -0.198757 & 0.198757 & 1.386121 & -3.103699 & 0.284924 & -2.376765 \\
\hline 138 & 60 & 0.959683 & 0.198757 & 0.198757 & 0.987375 & -3.007326 & 0.284924 & -0.764828 \\
\hline 139 & 61 & 0.980785 & -0.195090 & 0.000000 & 1.374447 & 3.141593 & 0.196349 & 3.141593 \\
\hline 140 & 62 & 0.980785 & 0.000000 & 0.195090 & 1.186043 & -3.060959 & 0.196349 & -1.570796 \\
\hline 141 & 63 & 0.980785 & 0.195090 & 0.000000 & 0.981748 & 3.141593 & 0.196349 & 0.000000 \\
\hline 142 & 64 & 1.000000 & 0.000000 & 0.000000 & 1.178097 & 3.141593 & 0.000000 & 0.000000 \\
\hline
\end{tabular}

Table 3: Sample normal and corresponding viewing and illumination angle for Camera Position 3 


\begin{tabular}{|c|c|c|c|c|c|c|c|c|}
\hline Image No. & Vertex & $n_{x}^{w}$ & $n_{y}^{w}$ & $n_{z}^{w}$ & $\theta_{r}$ & $\phi_{r}$ & $\theta_{i}$ & $\phi_{i}$ \\
\hline 143 & 3 & 0.195090 & 0.980785 & 0.000000 & 0.196349 & 3.141593 & 1.374447 & 0.000000 \\
\hline 144 & 6 & 0.198757 & 0.198757 & 0.959683 & 1.370707 & -2.376765 & 1.370707 & -0.764828 \\
\hline 145 & 7 & 0.198757 & 0.959683 & 0.198757 & 0.284924 & -2.376765 & 1.370707 & -0.041141 \\
\hline 146 & 10 & 0.208847 & 0.404615 & 0.890320 & 1.154239 & -2.096192 & 1.360401 & -0.430767 \\
\hline 147 & 11 & 0.208847 & 0.890320 & 0.404615 & 0.472749 & -2.096192 & 1.360401 & -0.094629 \\
\hline 148 & 14 & 0.211325 & 0.577350 & 0.788675 & 0.955317 & -2.005315 & 1.357866 & -0.281035 \\
\hline 149 & 15 & 0.211325 & 0.788675 & 0.577350 & 0.662145 & -2.005315 & 1.357866 & -0.153484 \\
\hline 150 & 18 & 0.382683 & 0.923880 & 0.000000 & 0.392699 & 3.141593 & 1.178098 & 0.000000 \\
\hline 151 & 21 & 0.404615 & 0.208847 & 0.890320 & 1.360401 & -2.710826 & 1.154239 & -1.045400 \\
\hline 152 & 22 & 0.404615 & 0.890320 & 0.208847 & 0.472749 & -2.710826 & 1.154239 & -0.094629 \\
\hline 153 & 24 & 0.408248 & 0.408248 & 0.816497 & 1.150262 & -2.456873 & 1.150262 & -0.684719 \\
\hline 154 & 26 & 0.408248 & 0.816497 & 0.408248 & 0.615479 & -2.456873 & 1.150262 & -0.201358 \\
\hline 155 & 28 & 0.426401 & 0.639603 & 0.639603 & 376815 & -2.376909 & 1.130287 & -0.403056 \\
\hline 156 & 31 & 0.555570 & 0.831470 & 0.000000 & 0.589048 & 3.141593 & 0.981748 & 0.000000 \\
\hline 157 & 34 & 0.577350 & 0.211325 & 0.788675 & 1.357866 & -2.860557 & 0.955317 & -1.136277 \\
\hline 158 & 35 & 0.577350 & 0.788675 & 0.211325 & 0.662145 & -2.860557 & 0.955317 & -0.153484 \\
\hline 159 & 37 & 0.639603 & 0.426401 & 0.639603 & 1.130287 & -2.738536 & 0.876815 & -0.764684 \\
\hline 160 & 39 & 0.639603 & 0.639603 & 0.426401 & 0.876815 & -2.738536 & 0.876815 & -0.403056 \\
\hline 161 & 42 & 0.707107 & 0.707107 & 0.000000 & 0.785398 & 3.141593 & 0.785398 & 0.000000 \\
\hline 162 & 45 & 0.788675 & 0.211325 & 0.577350 & 1.357866 & -2.988109 & 0.662145 & -1.136277 \\
\hline 163 & 46 & 0.788675 & 0.577350 & 0.211325 & 0.955317 & -2.988109 & 0.662145 & -0.281035 \\
\hline 164 & 48 & 0.816497 & 0.408248 & 0.408248 & 1.150262 & -2.940235 & 0.615479 & -0.684719 \\
\hline 165 & 51 & 0.831470 & 0.555570 & 0.000000 & 0.981748 & 3.141593 & 0.589048 & 0.000000 \\
\hline 166 & 54 & 0.890320 & 0.208847 & 0.404615 & 1.360401 & -3.046963 & 0.472749 & -1.045400 \\
\hline 167 & 55 & 0.890320 & 0.404615 & 0.208847 & 1.154239 & -3.046963 & 0.472749 & -0.430767 \\
\hline 168 & 58 & 0.923880 & 0.382683 & 0.000000 & 1.178098 & 3.141593 & 0.392699 & 0.000000 \\
\hline 169 & 60 & 0.959683 & 0.198757 & 0.198757 & 1.370707 & -3.100452 & 0.284924 & -0.764828 \\
\hline 170 & 63 & 0.980785 & 0.195090 & 0.000000 & 1.374447 & 3.141593 & 0.196349 & 0.000000 \\
\hline
\end{tabular}

Table 4: Sample normal and corresponding viewing and illumination angle for Camera Position 4

\begin{tabular}{|c|c|c|c|c|c|c|c|c|}
\hline Image No. & Vertex & $n_{x}^{w}$ & $n_{y}^{w}$ & $n_{z}^{w}$ & $\theta_{r}$ & $\phi_{r}$ & $\theta_{i}$ & $\phi_{i}$ \\
\hline 171 & 3 & 0.195090 & 0.980785 & 0.000000 & 0.589048 & 3.141593 & 1.374447 & 0.000000 \\
172 & 6 & 0.198757 & 0.198757 & 0.959683 & 1.463021 & -2.763231 & 1.370707 & -0.764828 \\
173 & 7 & 0.198757 & 0.959683 & 0.198757 & 0.625671 & -2.857075 & 1.370707 & -0.041141 \\
174 & 10 & 0.208847 & 0.404615 & 0.890320 & 1.272499 & -2.496589 & 1.360401 & -0.430767 \\
175 & 11 & 0.208847 & 0.890320 & 0.404615 & 0.733813 & -2.628785 & 1.360401 & -0.094629 \\
176 & 14 & 0.211325 & 0.577350 & 0.788675 & 1.101194 & -2.432726 & 1.357866 & -0.281035 \\
177 & 15 & 0.211325 & 0.788675 & 0.577350 & 0.866142 & -2.496546 & 1.357866 & -0.153484 \\
178 & 18 & 0.382683 & 0.923880 & 0.000000 & 0.785398 & 3.141593 & 1.178098 & 0.000000 \\
179 & 22 & 0.404615 & 0.890320 & 0.208847 & 0.839669 & -2.948852 & 1.154239 & -0.094629 \\
180 & 24 & 0.408248 & 0.408248 & 0.816497 & 1.348016 & -2.815465 & 1.150262 & -0.684719 \\
181 & 26 & 0.408248 & 0.816497 & 0.408248 & 0.929649 & -2.801295 & 1.150262 & -0.201358 \\
182 & 28 & 0.426401 & 0.639603 & 0.639603 & 1.128806 & -2.736898 & 1.130287 & -0.403056 \\
183 & 31 & 0.555570 & 0.831470 & 0.000000 & 0.981747 & 3.141593 & 0.981748 & 0.000000 \\
184 & 35 & 0.577350 & 0.788675 & 0.211325 & 1.038285 & -3.013835 & 0.955317 & -0.153484 \\
185 & 37 & 0.639603 & 0.426401 & 0.639603 & 1.421060 & -3.015733 & 0.876815 & -0.764684 \\
186 & 39 & 0.639603 & 0.639603 & 0.426401 & 1.217332 & -2.966775 & 0.876815 & -0.403056 \\
187 & 42 & 0.707107 & 0.707107 & 0.000000 & 1.178097 & 3.141593 & 0.785398 & 0.000000 \\
188 & 46 & 0.788675 & 0.577350 & 0.211325 & 1.337086 & -3.090099 & 0.662145 & -0.281035 \\
189 & 51 & 0.831470 & 0.555570 & 0.000000 & 1.374447 & 3.141593 & 0.589048 & 0.000000 \\
\hline
\end{tabular}

Table 5: Sample normal and corresponding viewing and illumination angle for Camera Position 5 


\begin{tabular}{|c|c|c|c|c|c|c|c|c|}
\hline Image No. & Vertex & $n_{x}^{w}$ & $n_{y}^{w}$ & $n_{z}^{w}$ & $\theta_{r}$ & $\phi_{r}$ & $\theta_{i}$ & $\phi_{i}$ \\
\hline 190 & 3 & 0.195090 & 0.980785 & 0.000000 & 0.981747 & 3.141593 & 1.374447 & 0.000000 \\
191 & 7 & 0.198757 & 0.959683 & 0.198757 & 1.002667 & -3.011773 & 1.370707 & -0.041141 \\
192 & 10 & 0.208847 & 0.404615 & 0.890320 & 1.431921 & -2.864769 & 1.360401 & -0.430767 \\
193 & 11 & 0.208847 & 0.890320 & 0.404615 & 1.068004 & -2.895807 & 1.360401 & -0.09462 \\
194 & 14 & 0.211325 & 0.577350 & 0.788675 & 1.308997 & -2.790714 & 1.357866 & -0.281035 \\
195 & 15 & 0.211325 & 0.788675 & 0.577350 & 1.150262 & -2.819842 & 1.357866 & -0.153484 \\
196 & 18 & 0.382683 & 0.923880 & 0.000000 & 1.178097 & 3.141593 & 1.178098 & 0.000000 \\
197 & 22 & 0.404615 & 0.890320 & 0.208847 & 1.220213 & -3.063418 & 1.154239 & -0.094629 \\
198 & 26 & 0.408248 & 0.816497 & 0.408248 & 1.277953 & -3.006341 & 1.150262 & -0.201358 \\
199 & 28 & 0.426401 & 0.639603 & 0.639603 & 1.419463 & -3.014362 & 1.130287 & -0.403056 \\
200 & 31 & 0.555570 & 0.831470 & 0.000000 & 1.374446 & 3.141593 & 0.981748 & 0.000000 \\
201 & 35 & 0.577350 & 0.788675 & 0.211325 & 1.420805 & -3.108912 & 0.955317 & -0.153484 \\
\hline
\end{tabular}

Table 6: Sample normal and corresponding viewing and illumination angle for Camera Position 6

\begin{tabular}{|c|c|c|c|c|c|c|c|c|}
\hline Image No. & Vertex & $n_{x}^{w}$ & $n_{y}^{w}$ & $n_{z}^{w}$ & $\theta_{r}$ & $\phi_{r}$ & $\theta_{i}$ & $\phi_{i}$ \\
\hline 202 & 3 & 0.195090 & 0.980785 & 0.000000 & 1.374447 & 3.141593 & 1.374447 & 0.000000 \\
203 & 7 & 0.198757 & 0.959683 & 0.198757 & 1.386121 & -3.103699 & 1.370707 & -0.041141 \\
204 & 11 & 0.208847 & 0.890320 & 0.404615 & 1.422492 & -3.075442 & 1.360401 & -0.094629 \\
205 & 15 & 0.211325 & 0.788675 & 0.577350 & 1.464020 & -3.065729 & 1.357866 & -0.153484 \\
\hline
\end{tabular}

Table 7: Sample normal and corresponding viewing and illumination angle for Camera Position 7

The translation vector $\mathbf{T}_{\mathbf{w}}^{\mathbf{i}}$ is used to translate from the world-frame origin to the image-frame origin. Let $\mathbf{R}_{\mathbf{s}}^{\mathbf{w}}$ denote the rotation matrix which transforms points from sample to world coordinates. For example if the sample is oriented so that it's normal is pointing along $\mathbf{x}_{\mathbf{w}}$, i.e. if the sample is frontal to the lamp then

$$
\mathbf{R}_{\mathbf{s}}^{\mathbf{w}}=\left[\begin{array}{ccc}
0 & 0 & 1 \\
-1 & 0 & 0 \\
0 & 1 & 0
\end{array}\right]
$$

The transformation of an arbitrary position vector $\mathbf{p}_{\mathbf{s}}$ from sample coordinates to image coordinates is given by

$$
\mathbf{p}_{\mathbf{i}}=\mathbf{R}_{\mathrm{s}}^{\mathrm{i}} \mathbf{p}_{\mathrm{s}}+\mathbf{T}_{\mathrm{w}}^{\mathrm{i}}
$$

where

$$
\mathbf{R}_{\mathrm{s}}^{\mathrm{i}}=\mathbf{R}_{\mathrm{w}}^{\mathrm{i}} \mathbf{R}_{\mathrm{s}}^{\mathrm{w}}
$$

Assuming orthographic projection and unity magnification, the $3 \times 1$ vector depicting a corner point $\mathbf{a}_{\mathbf{s}}$ in sample coordinate frames is related to the $2 \times 1$ vector $\mathbf{a}_{\mathbf{i}}$ as follows

$$
\mathbf{a}_{\mathbf{i}}=\mathbf{R}_{\mathrm{s}}^{\mathbf{i}^{\prime}} \mathbf{a}_{\mathbf{s}}+\mathbf{T}_{\mathbf{w}}^{\mathbf{i}^{\prime}}
$$

where $\mathbf{R}_{\mathrm{s}}^{\mathrm{i}^{\prime}}$ is the first two rows of $\mathbf{R}_{\mathbf{s}}^{\mathrm{i}}$ and $\mathbf{T}_{\mathrm{w}}^{\mathrm{i}^{\prime}}$ is the first two rows of $\mathbf{T}_{\mathrm{w}}^{\mathrm{i}}$. This relation is the basis for the linear regression which uses manually chosen values of $\mathbf{a}_{\mathbf{i}}, \mathbf{b}_{\mathbf{i}}, \mathbf{c}_{\mathbf{i}}, \mathbf{d}_{\mathbf{i}}$ for several camera/sample positions and the corresponding known values of $\mathbf{R}_{\mathbf{s}}^{\mathbf{i}^{\prime}}$ to estimate $\mathbf{a}_{\mathbf{s}}, \mathbf{b}_{\mathbf{s}}, \mathbf{c}_{\mathbf{s}}, \mathbf{d}_{\mathbf{s}}$. Once these corner points are estimated in the sample coordinate frame, Equation 18 is used again to predict the position of corner points for the remaining images.

\section{B.2 ESTIMATION OF CORNER LOCATIONS}

In Equation 18, the only unknowns are the corner locations and $\mathbf{T}_{\mathbf{w}}^{\mathbf{i}^{\prime}}$. Ideally the translation from world to image origin would be fixed; however significant shifts were noticed for each camera position. To compensate for these shifts a different value for $\mathbf{T}_{\mathbf{w}}^{\mathbf{i}^{\prime}}$ is estimated for each camera position 
for a total of 14 translational parameters. There are four unknown $3 \times 1$ corner vectors. If we assume that the thickness of the sample is approximately uniform than each corner vector has the same component along $\mathbf{z}_{\mathbf{s}}$. Consequently, there are 9 corner parameters to be estimated. In total 23 parameters need to be estimated. Define

$$
\mathbf{R}=\left[\begin{array}{ll}
\mathbf{R}_{\mathbf{s}}^{\mathbf{i}^{\prime}}(1,1) & \mathbf{R}_{\mathbf{s}}^{\mathbf{i}^{\prime}}(1,2) \\
\mathbf{R}_{\mathbf{s}}^{\mathbf{i}^{\prime}}(2,1) & \mathbf{R}_{\mathbf{s}}^{\mathbf{i}^{\prime}}(2,2)
\end{array}\right]
$$

where $\mathbf{R}_{\mathbf{s}}^{\mathrm{i}^{\prime}}(1, \mathrm{~m})$ is the value in the lth row and mth column of $\mathbf{R}_{\mathbf{s}}^{\mathrm{i}^{\prime}}$,

$$
\begin{gathered}
\mathbf{r}=\left[\begin{array}{l}
\mathbf{R}_{\mathbf{s}}^{\mathbf{i}^{\prime}}(1,3) \\
\mathbf{R}_{\mathbf{s}}^{\mathbf{i}^{\prime}}(2,3)
\end{array}\right], \\
\overline{\mathbf{0}}=\left[\begin{array}{ll}
0 & 0 \\
0 & 0
\end{array}\right], \\
\mathbf{I}_{\mathbf{0}}=\left[\begin{array}{ll}
1 & 0 \\
0 & 1
\end{array}\right],
\end{gathered}
$$

and

$$
\begin{aligned}
& \mathbf{I}_{1}=\left[\begin{array}{llllllll}
\mathbf{I} & \overline{\mathbf{0}} & \overline{\mathbf{0}} & \overline{\mathbf{0}} & \overline{\mathbf{0}} & \overline{\mathbf{0}} & \overline{\mathbf{0}} & \overline{\mathbf{0}}
\end{array}\right] \\
& \mathbf{I}_{2}=\left[\begin{array}{llllllll}
\overline{\mathbf{0}} & \mathbf{I} & \overline{\mathbf{0}} & \overline{\mathbf{0}} & \overline{\mathbf{0}} & \overline{\mathbf{0}} & \overline{\mathbf{0}} & \overline{\mathbf{0}}
\end{array}\right] \\
& \mathbf{I}_{7}=\left[\begin{array}{llllllll}
\overline{\mathbf{0}} & \overline{\mathbf{0}} & \overline{\mathbf{0}} & \overline{\mathbf{0}} & \overline{\mathbf{0}} & \overline{\mathbf{0}} & \overline{\mathbf{0}} & \mathrm{I}
\end{array}\right] .
\end{aligned}
$$

Let $j \in\{1 . .7\}$ denote the camera position and let $n \in\{1 . . N\}$ denote the image index where $N$ is the total number of images that had corners manually chosen. Define

$$
\mathbf{A}_{n}=\left[\begin{array}{cccccc}
\mathbf{R} & \overline{\mathbf{0}} & \overline{\mathbf{0}} & \overline{\mathbf{0}} & \mathbf{r} & \mathbf{I}_{j} \\
\overline{\mathbf{0}} & \mathbf{R} & \overline{\mathbf{0}} & \overline{\mathbf{0}} & \mathbf{r} & \mathbf{I}_{j} \\
\overline{\mathbf{0}} & \overline{\mathbf{0}} & \mathbf{R} & \overline{\mathbf{0}} & \mathbf{r} & \mathbf{I}_{j} \\
\overline{\mathbf{0}} & \overline{\mathbf{0}} & \overline{\mathbf{0}} & \mathbf{R} & \mathbf{r} & \mathbf{I}_{j}
\end{array}\right]
$$

and

$$
\mathbf{A}=\left[\begin{array}{llll}
\mathbf{A}_{1} & \mathbf{A}_{2} & \ldots & \mathbf{A}_{N}
\end{array}\right]^{T}
$$

Define the right hand side vector

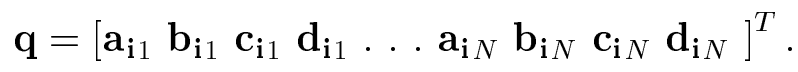

The solution vector $\mathbf{u}$ is

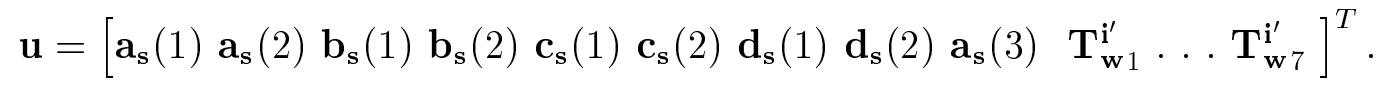

The problem is to solve for $\mathbf{u}$ in the equation

$$
\mathrm{Au}=\mathbf{q}
$$

where $\mathbf{A}$ is an $8 N \times 23$ matrix and $\mathbf{q}$ is an $8 N \times 1$ matrix. The least square error solution is given by

$$
\hat{\mathbf{u}}=\left(\mathbf{A}^{T} \mathbf{A}\right)^{-1} \mathbf{A}^{T} \mathbf{q} .
$$

where $\mathbf{A}^{T} \mathbf{A}$ is a $23 \times 23$ matrix, $\mathbf{A}^{T} \mathbf{q}$ is a $23 \times 1$ vector and $\hat{\mathbf{u}}$ is the $23 \times 1$ solution vector.

There were some observed errors in the positioning of some corner points. Therefore manual adjustment of a few corner points was necessary so that the segmented image would include only sample data. 


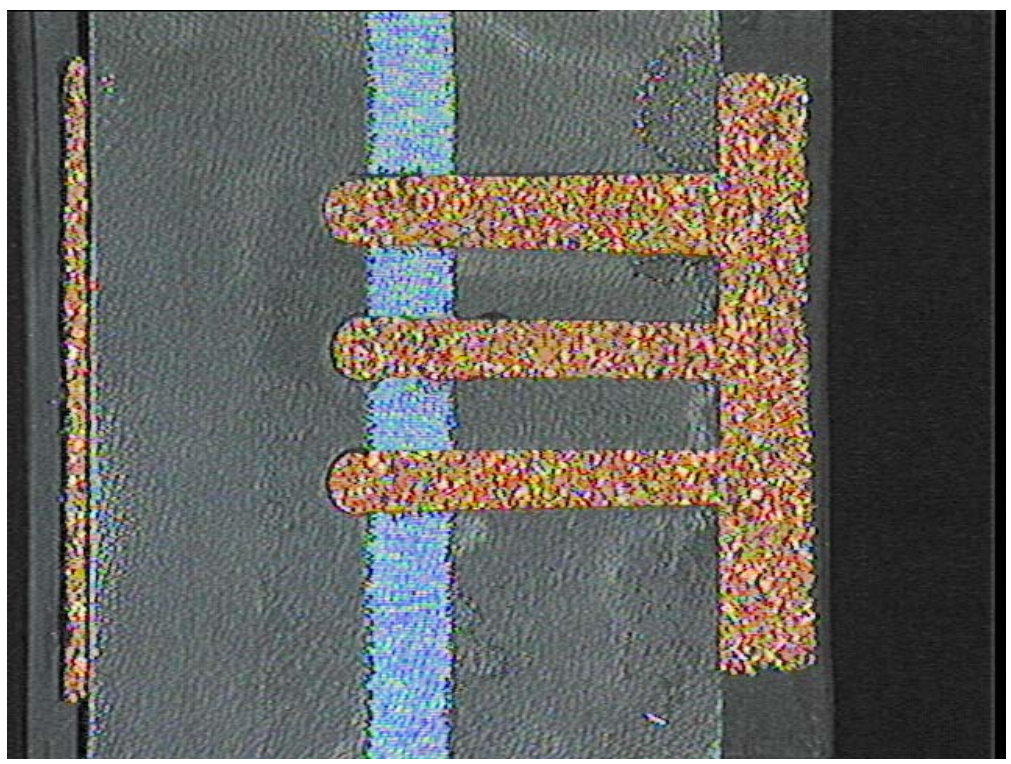

Figure 15: Fiducial Marker attached to Sample. This metallic grid with painted stripe is used as a fiducial marker to specify a spatial region for measuring radiance with the photometer and recording pixel values with the video camera. These radiance and pixel measurements are used in radiometric calibration.

\section{RADIOMETRIC CALIBRATION}

Radiometric calibration is performed to interpret pixel values as radiance. This calibration is done by measuring radiance from the samples and from a Kodak Standard Color Card (illustrated in Figure 16) using a spectrometer ${ }^{8}$. We refer to the radiance measurements and pixel values from the standard color card as the standard calibration data. The radiance measurements and pixel values from the sample is termed sample calibration data.

Obtaining the standard calibration data is straight forward. The photometer's region of interest is positioned within each "strip" on the card shown in Figure 16. The photometer records 200 radiance values, corresponding to the radiance in watts/(steridian-meter ${ }^{2}$ ) for each $2 \mathrm{~nm}$ interval between $380 \mathrm{~nm}$ and $780 \mathrm{~nm}$. After the aperture and video-camera setting are adjusted for each sample, an image is recorded of the standard color card. The pixels in each "strip" are averaged. Since there are 40 strips on the card (20 gray and 12 color), the standard calibration data is 40 radiance values with corresponding pixel values. Note that during data collection, the standard color card had to be imaged for every sample (since the camera's aperture was typically adjusted for each sample). The photometer recordings are a function of the light source and was not repeated for every sample.

To obtain the sample calibration data, a fiducial marker is necessary to locate a region where pixel values and photometer readings could be obtained. We use a metal grid that fit over the sample as the fiducial marker as illustrated in Figure 15. The circular photometer region $(0.8 \mathrm{~cm}$ diameter) is positioned so that its center diameter aligns with the right edge of the grid line. For 8 sample orientations, 3 radiance and pixel values are obtained corresponding to the 3 slit openings of the grid. The 8 sample orientations correspond to the light source direction and the directions of camera position 1 through 7 . These orientations were chosen so that bright and dark points would be included in the sample calibration data.

\footnotetext{
${ }^{8}$ SpectraScan PR-704 by Photoresearch (Chatsworth,CA).
} 


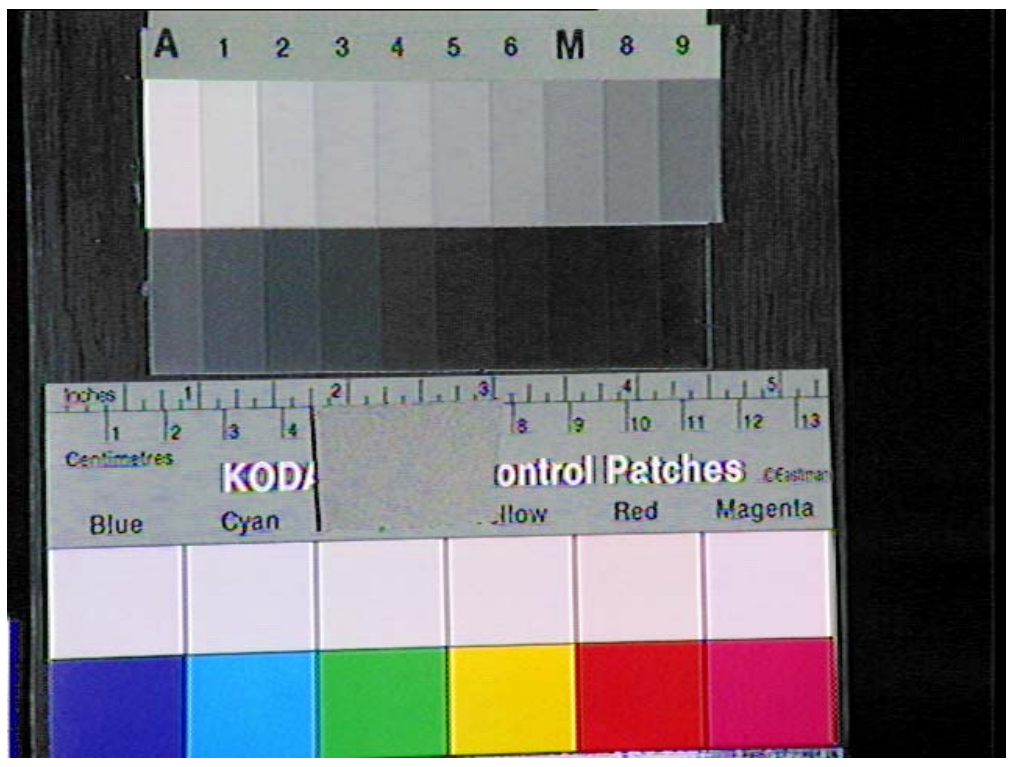

Figure 16: Kodak Standard Color Card with 20 strips of gray and 12 color strips.

We use two methods for radiometric calibration. In both methods the relationship between radiance and associated pixel value is approximated as linear. The first method, termed gray calibration, relates the average of the red, green and blue pixel to the total radiance recorded by the photometer (i.e. radiance from the wavelength range $380 \mathrm{~nm}-780 \mathrm{~nm}$ ). Letting $r$ denote the total radiance and $p$ denote the average pixel value, the parameters estimated are $a$ and $b$ where

$$
r=a * p+b
$$

The gray calibration results are illustrated in Figures 18 and 19. The resulting calibration parameters for this method are as follows:

The second method, termed color calibration, estimates the camera sensitivity curves for the red, green and blue CCD of the video camera. The average radiance for the red, green and blue spectral regions is obtained by integrating the estimated sensitivity curves multiplied by the radiance per wavelength (as measured by the photometer). The relationship between the the $R / G / B$ pixels and corresponding $\mathrm{R} / \mathrm{G} / \mathrm{B}$ radiance is assumed linear. The sensitivity function is assumed to be a unit area guassian function. For each sample, the mean and variance of this guassian function is estimated along with the linear radiometric calibration curves through nonlinear estimation. The resulting mean and variances over all samples are averaged to obtain the final estimates of the sensitivity curves. These estimated sensitivity curves, depicted in Figure 17, were used to obtain the final estimate for the linear relationships between the $\mathrm{R} / \mathrm{G} / \mathrm{B}$ pixel values and $\mathrm{R} / \mathrm{G} / \mathrm{B}$ radiance for each sample. Let $r_{r}, r_{g}$ and $r_{b}$ denote the red, green and blue radiance, respectively and let $p_{r}, p_{g}$ and $p_{b}$ denot the red, green and blue pixel values respectively. Then the color calibration method estimates $a_{r}, b_{r}, a_{g}, b_{g}, a_{b}$, and $b_{b}$, where

$$
\begin{aligned}
& r_{r}=a_{r} * p_{r}+b_{r} \\
& r_{g}=a_{g} * p_{g}+b_{g} \\
& r_{b}=a_{b} * p_{b}+b_{b}
\end{aligned}
$$

The color calibration results are illustrated in Figures 20, 21, 22, 23, 24, and 25. The resulting calibration parameters for the color calibration method are as follows: 


\begin{tabular}{|c|c|c|}
\hline Sample Number & $a$ & $b$ \\
\hline 1 & 0.007646 & -0.092646 \\
\hline 2 & 0.007697 & -0.057489 \\
\hline 3 & 0.011687 & -0.089474 \\
\hline 4 & 0.008654 & -0.196334 \\
\hline 5 & 0.010682 & -0.054209 \\
\hline 6 & 0.007561 & -0.081635 \\
\hline 7 & 0.008403 & -0.005292 \\
\hline 8 & 0.011256 & -0.134361 \\
\hline 9 & 0.075967 & -0.595488 \\
\hline 10 & 0.081855 & -0.591199 \\
\hline 11 & 0.074773 & -0.896702 \\
\hline 12 & 0.082896 & -0.401243 \\
\hline 13 & 0.021683 & -0.297281 \\
\hline 14 & 0.065336 & -0.937571 \\
\hline 15 & 0.236777 & -2.373478 \\
\hline 16 & 0.066489 & -0.776715 \\
\hline 17 & 0.102022 & -0.326226 \\
\hline 18 & 0.082454 & -0.624636 \\
\hline 19 & 0.014382 & -0.082942 \\
\hline 20 & 0.066848 & 0.341182 \\
\hline 21 & 0.047135 & 0.017802 \\
\hline 22 & 0.051874 & -0.109838 \\
\hline 23 & 0.066701 & -0.277704 \\
\hline 24 & 0.056012 & -0.773515 \\
\hline 25 & 0.044797 & 0.077123 \\
\hline 26 & 0.083199 & -0.550297 \\
\hline 27 & 0.113167 & -0.648234 \\
\hline 28 & 0.063683 & -0.423375 \\
\hline 29 & 0.064007 & -0.549698 \\
\hline 30 & 0.073835 & -0.453216 \\
\hline 31 & 0.076897 & -0.386471 \\
\hline 32 & 0.050812 & -0.266455 \\
\hline 33 & 0.015020 & -0.135932 \\
\hline 34 & 0.048566 & -0.191045 \\
\hline 35 & 0.064377 & -0.283413 \\
\hline 36 & 0.060800 & -0.375083 \\
\hline 37 & 0.031799 & -0.246173 \\
\hline 38 & 0.079932 & -0.815990 \\
\hline 39 & 0.053476 & -0.068293 \\
\hline 40 & 0.055310 & -0.267601 \\
\hline 41 & 0.036139 & -0.009112 \\
\hline 42 & 0.058214 & -0.219933 \\
\hline 43 & 0.048633 & -0.157401 \\
\hline 44 & 0.061965 & -0.193188 \\
\hline 45 & 0.019034 & -0.113938 \\
\hline 46 & 0.016155 & -0.176481 \\
\hline 47 & 0.022797 & -0.133047 \\
\hline 48 & 0.012764 & -0.021903 \\
\hline 49 & 0.018468 & -0.185967 \\
\hline 50 & 0.020797 & -0.124803 \\
\hline 51 & 0.019157 & -0.109284 \\
\hline 52 & 0.017870 & -0.101733 \\
\hline 53 & 0.011529 & -0.042242 \\
\hline 54 & 0.009872 & -0.074923 \\
\hline 55 & 0.025887 & 0.007188 \\
\hline 56 & 0.021053 & -0.323061 \\
\hline 57 & 0 & 0 \\
\hline 58 & 0 & 0 \\
\hline 59 & 0.027655 & -0.625534 \\
\hline 60 & 0.020467 & -0.036492 \\
\hline 61 & 0.002498 & -0.082089 \\
\hline
\end{tabular}

Table 8: Parameters from Gray Calibration 


\begin{tabular}{|c|c|c|c|c|c|c|}
\hline Sample Number & $a_{r}$ & $b_{r}$ & $a_{g}$ & $b_{g}$ & $a_{b}$ & $b_{b}$ \\
\hline 1 & 0.000025 & -0.000044 & 0.000021 & -0.000170 & 0.000009 & 0.000002 \\
\hline 2 & 0.000022 & 0.000130 & 0.000020 & -0.000148 & 0.000008 & 0.000028 \\
\hline 3 & 0.000035 & -0.000124 & 0.000028 & -0.000174 & 0.000013 & -0.000109 \\
\hline 4 & 0.000024 & -0.000112 & 0.000021 & -0.000278 & 0.000008 & 0.000010 \\
\hline 5 & 0.000031 & -0.000058 & 0.000027 & -0.000218 & 0.000012 & -0.000049 \\
\hline 6 & 0.000018 & 0.000150 & 0.000018 & -0.000196 & 0.000006 & 0.000077 \\
\hline 7 & 0.000004 & -0.000006 & 0.000005 & -0.000016 & 0.000002 & -0.000030 \\
\hline 8 & 0.000024 & 0.000025 & 0.000023 & -0.000369 & 0.000010 & -0.000069 \\
\hline 9 & 0.000213 & -0.001408 & 0.000139 & -0.001049 & 0.000067 & -0.000497 \\
\hline 10 & 0.000242 & -0.001194 & 0.000157 & -0.000528 & 0.000075 & -0.000318 \\
\hline 11 & 0.000218 & -0.001021 & 0.000141 & -0.000232 & 0.000068 & -0.000173 \\
\hline 12 & 0.000232 & -0.000642 & 0.000152 & -0.000426 & 0.000072 & -0.000203 \\
\hline 13 & 0.000061 & -0.000582 & 0.000040 & -0.000503 & 0.000020 & -0.000272 \\
\hline 14 & 0.000170 & -0.002303 & 0.000127 & -0.002200 & 0.000062 & -0.001022 \\
\hline 15 & 0.000572 & -0.003279 & 0.000416 & -0.004377 & 0.000184 & -0.001264 \\
\hline 16 & 0.000092 & 0.000442 & 0.000097 & -0.002184 & 0.000043 & -0.000662 \\
\hline 17 & 0.000289 & -0.000751 & 0.000197 & -0.000986 & 0.000092 & -0.000431 \\
\hline 18 & 0.000210 & -0.000320 & 0.000143 & -0.000710 & 0.000067 & -0.000370 \\
\hline 19 & 0.000042 & -0.000321 & 0.000029 & -0.000083 & 0.000015 & -0.000157 \\
\hline 20 & 0.000212 & -0.000210 & 0.000146 & -0.000642 & 0.000068 & -0.000233 \\
\hline 21 & 0.000096 & 0.000925 & 0.000099 & -0.001918 & 0.000044 & -0.000624 \\
\hline 22 & 0.000129 & -0.000134 & 0.000108 & -0.001682 & 0.000055 & -0.000800 \\
\hline 23 & 0.000162 & -0.002388 & 0.000118 & -0.002695 & 0.000057 & -0.000844 \\
\hline 24 & 0.000104 & 0.001151 & 0.000082 & -0.000570 & 0.000045 & -0.000614 \\
\hline 25 & 0.000087 & -0.000131 & 0.000079 & -0.001003 & 0.000037 & -0.000396 \\
\hline 26 & 0.000206 & -0.001284 & 0.000143 & -0.001305 & 0.000067 & -0.000589 \\
\hline 27 & 0.000363 & -0.004614 & 0.000251 & -0.003889 & 0.000114 & -0.001303 \\
\hline 28 & 0.000180 & -0.000416 & 0.000123 & -0.000548 & 0.000057 & -0.000209 \\
\hline 29 & 0.000184 & -0.000565 & 0.000126 & -0.000930 & 0.000057 & -0.000125 \\
\hline 30 & 0.000209 & -0.000465 & 0.000143 & -0.000843 & 0.000065 & -0.000090 \\
\hline 31 & 0.000217 & -0.000615 & 0.000144 & -0.000638 & 0.000067 & -0.000184 \\
\hline 32 & 0.000147 & -0.001754 & 0.000100 & -0.001338 & 0.000048 & -0.000687 \\
\hline 33 & 0.000049 & -0.000370 & 0.000031 & -0.000280 & 0.000015 & -0.000171 \\
\hline 34 & 0.000172 & -0.001325 & 0.000120 & -0.001423 & 0.000053 & -0.000398 \\
\hline 35 & 0.000211 & -0.004222 & 0.000138 & -0.002689 & 0.000063 & -0.001150 \\
\hline 36 & 0.000167 & -0.001144 & 0.000116 & -0.001227 & 0.000049 & -0.000277 \\
\hline 37 & 0.000098 & -0.001567 & 0.000069 & -0.001101 & 0.000036 & -0.000766 \\
\hline 38 & 0.000219 & -0.001377 & 0.000146 & -0.000985 & 0.000068 & -0.000359 \\
\hline 39 & 0.000053 & -0.000241 & 0.000041 & -0.000363 & 0.000024 & -0.000211 \\
\hline 40 & 0.000126 & -0.000688 & 0.000086 & -0.000765 & 0.000043 & -0.000376 \\
\hline 41 & 0.000117 & -0.000705 & 0.000091 & -0.001343 & 0.000041 & -0.000527 \\
\hline 42 & 0.000144 & 0.000573 & 0.000107 & -0.000610 & 0.000049 & -0.000260 \\
\hline 43 & 0.000166 & -0.001118 & 0.000109 & -0.000802 & 0.000048 & -0.000293 \\
\hline 44 & 0.000148 & -0.001217 & 0.000113 & -0.001551 & 0.000045 & -0.000432 \\
\hline 45 & 0.000056 & -0.000475 & 0.000043 & -0.000468 & 0.000024 & -0.000259 \\
\hline 46 & 0.000046 & -0.000205 & 0.000036 & -0.000337 & 0.000020 & -0.000121 \\
\hline 47 & 0.000068 & -0.000693 & 0.000052 & -0.000679 & 0.000030 & -0.000431 \\
\hline 48 & 0.000029 & -0.000007 & 0.000031 & -0.000606 & 0.000017 & -0.000238 \\
\hline 49 & 0.000053 & -0.000241 & 0.000041 & -0.000363 & 0.000024 & -0.000211 \\
\hline 50 & 0.000061 & -0.000739 & 0.000047 & -0.000688 & 0.000027 & -0.000343 \\
\hline 51 & 0.000048 & -0.000140 & 0.000036 & -0.000231 & 0.000022 & -0.000196 \\
\hline 52 & 0.000050 & -0.000355 & 0.000038 & -0.000385 & 0.000022 & -0.000209 \\
\hline 53 & 0.000020 & -0.000287 & 0.000013 & -0.000107 & 0.000009 & -0.000167 \\
\hline 54 & 0.000016 & -0.000080 & 0.000013 & -0.000104 & 0.000008 & -0.000095 \\
\hline 55 & 0.000045 & -0.000077 & 0.000035 & 0.000002 & 0.000021 & -0.000093 \\
\hline 56 & 0.000040 & -0.000049 & 0.000032 & -0.000270 & 0.000020 & -0.000181 \\
\hline 57 & 0 & 0 & 0 & 0 & 0 & 0 \\
\hline 58 & 0 & 0 & 0 & 0 & 0 & 0 \\
\hline 59 & 0.000044 & -0.000416 & 0.000034 & -0.000411 & 0.000021 & -0.000287 \\
\hline 60 & 0.000032 & 0.000103 & 0.000026 & -0.000083 & 0.000016 & -0.000111 \\
\hline 61 & 0.000006 & -0.000089 & 0.000005 & -0.000063 & 0.000003 & -0.000054 \\
\hline
\end{tabular}

Table 9: Parameters from Color Calibration 


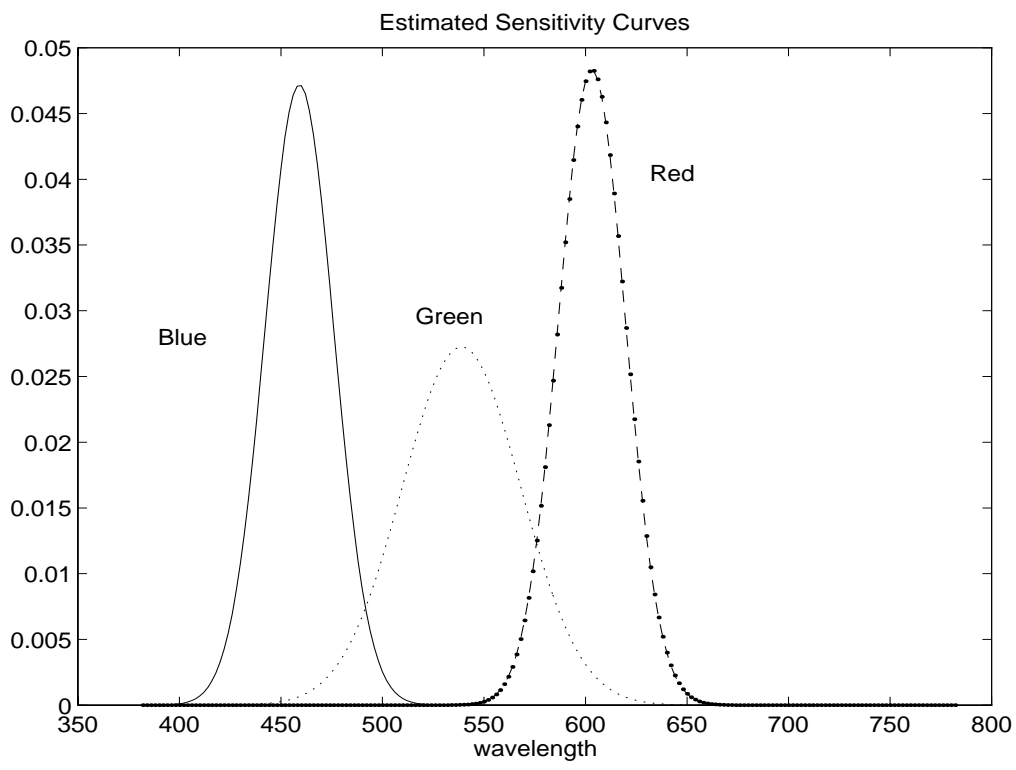

Figure 17: The estimated sensitivity curve for the 3CCD color video camera. The mean and std for the red sensitivity curve were $604 \mathrm{~nm}$ and $16.6 \mathrm{~nm}$, respectively. The mean and std for the green sensitivity curve were $538 \mathrm{~nm}$ and $29.2 \mathrm{~nm}$, respectively. The mean and std for the red sensitivity curve were $460 \mathrm{~nm}$ and $17 \mathrm{~nm}$, respectively. Note that each estimated sensitivity curve is normalized to have unit area.

In both the gray and color calibration methods the data is not always weighted equally. Sample calibration data is weighted more than the standard calibration except when the sample calibration data suffers from underflow or overflow. Data with significant pixel underflow (pixel values near zero) or overflow (pixel values near 255) are not used. 

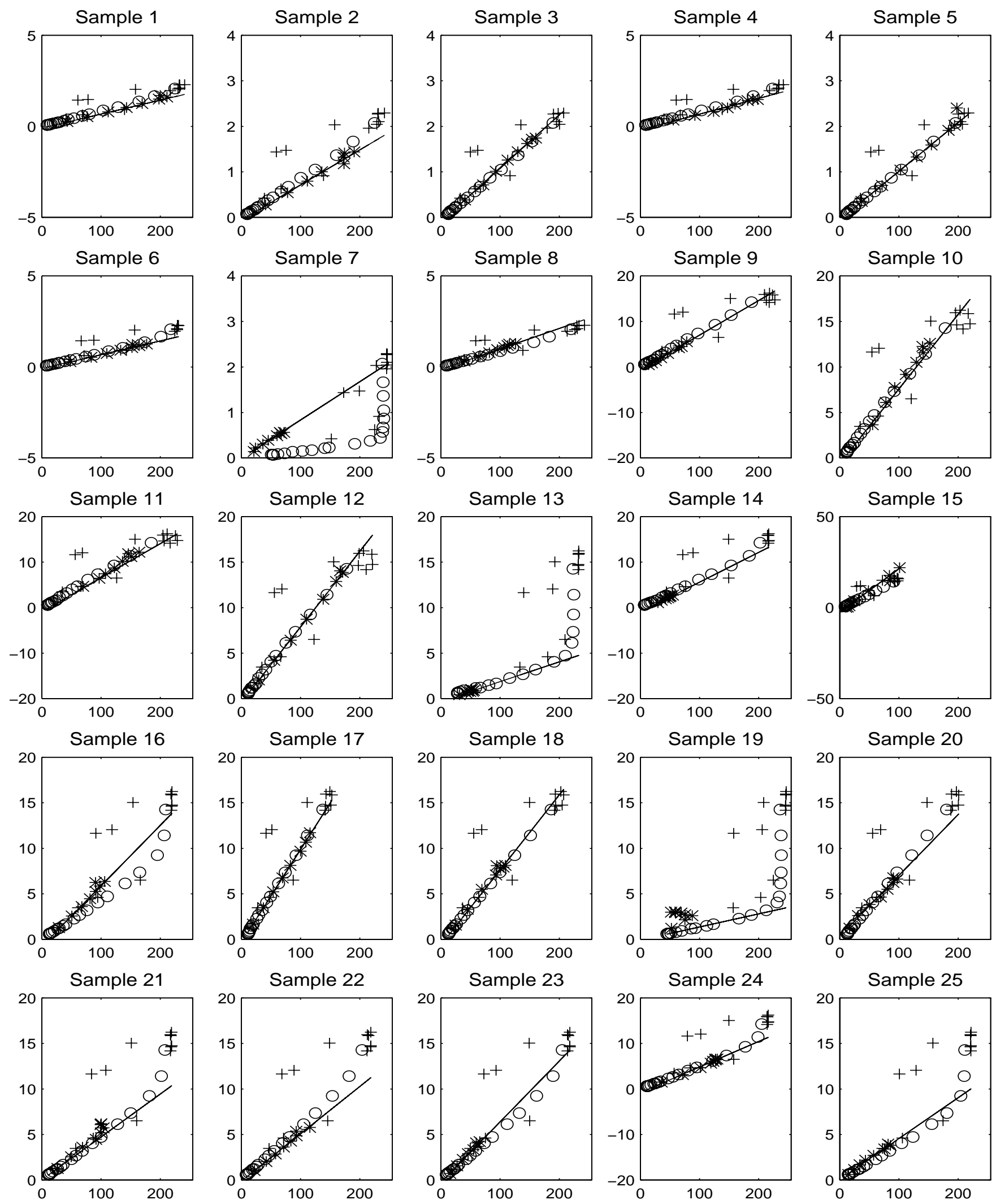

Figure 18: Calibration plots for the gray calibration data for samples 1-25. The sample calibration data are plotted with the symbol "*". The gray strips from the standard calibration data are plotted with the symbol "o", and the colored strips from the standard calibration data are plotted with the symbol "+". The estimated linear calibration function is plotted with a solid line. Figures 19, $20,21,22,23,24$, and 25 use the same plotting symbols. 

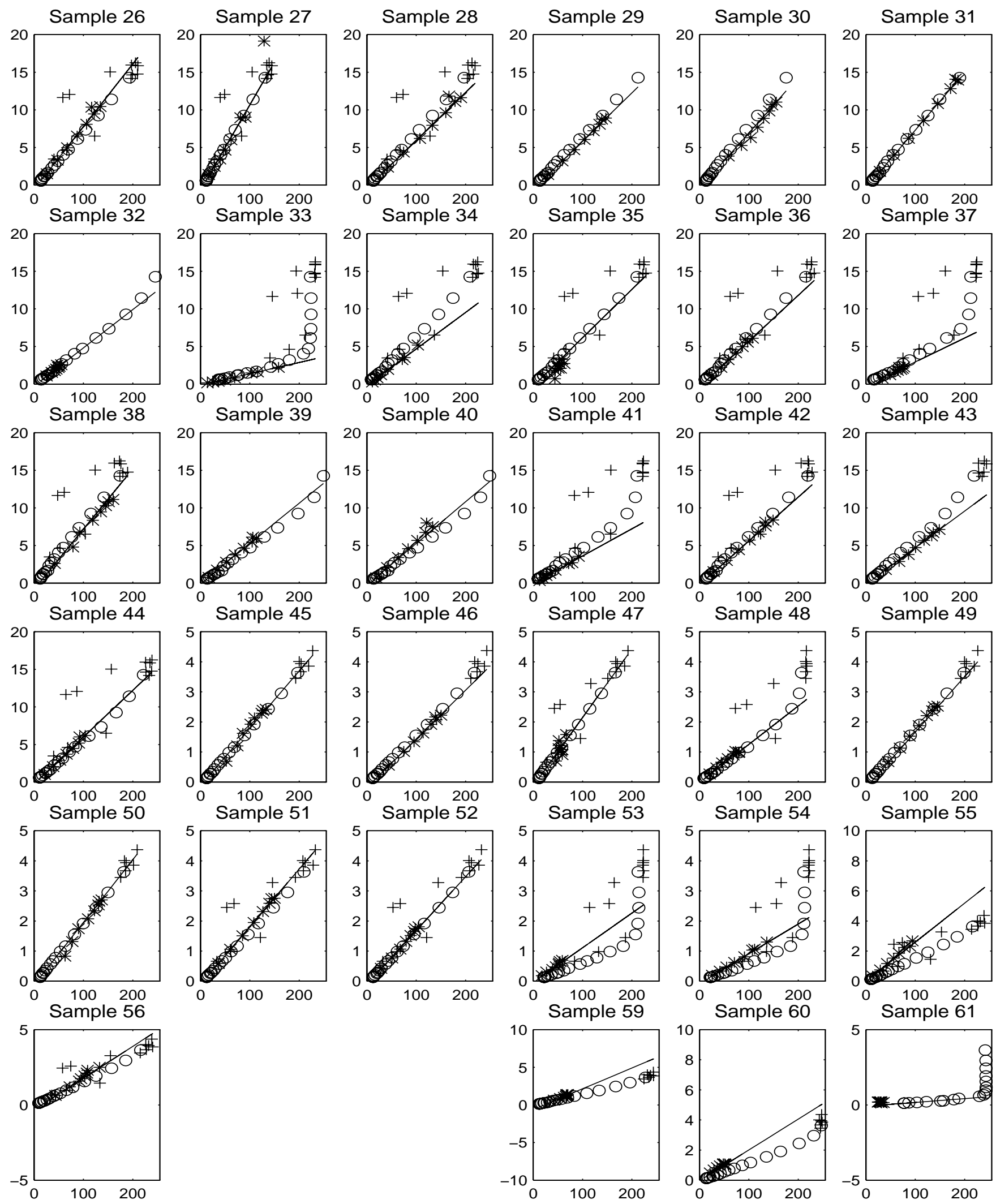

Figure 19: Calibration plots for the gray calibration data for samples 26-61. The plotting symbols are described in Figure 18. 

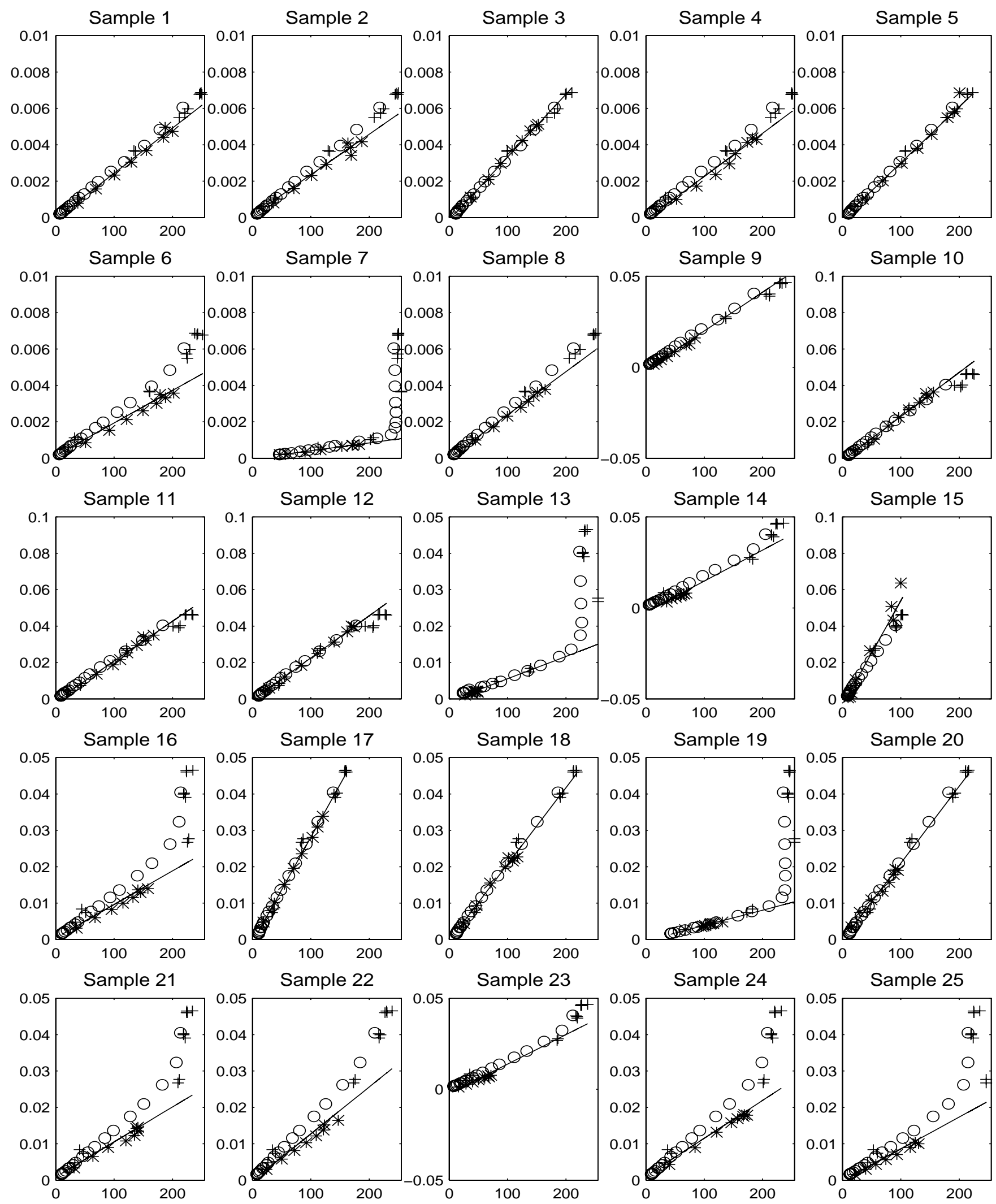

Figure 20: Calibration plots for the red calibration data for samples 1-25. The plotting symbols are described in Figure 18. 

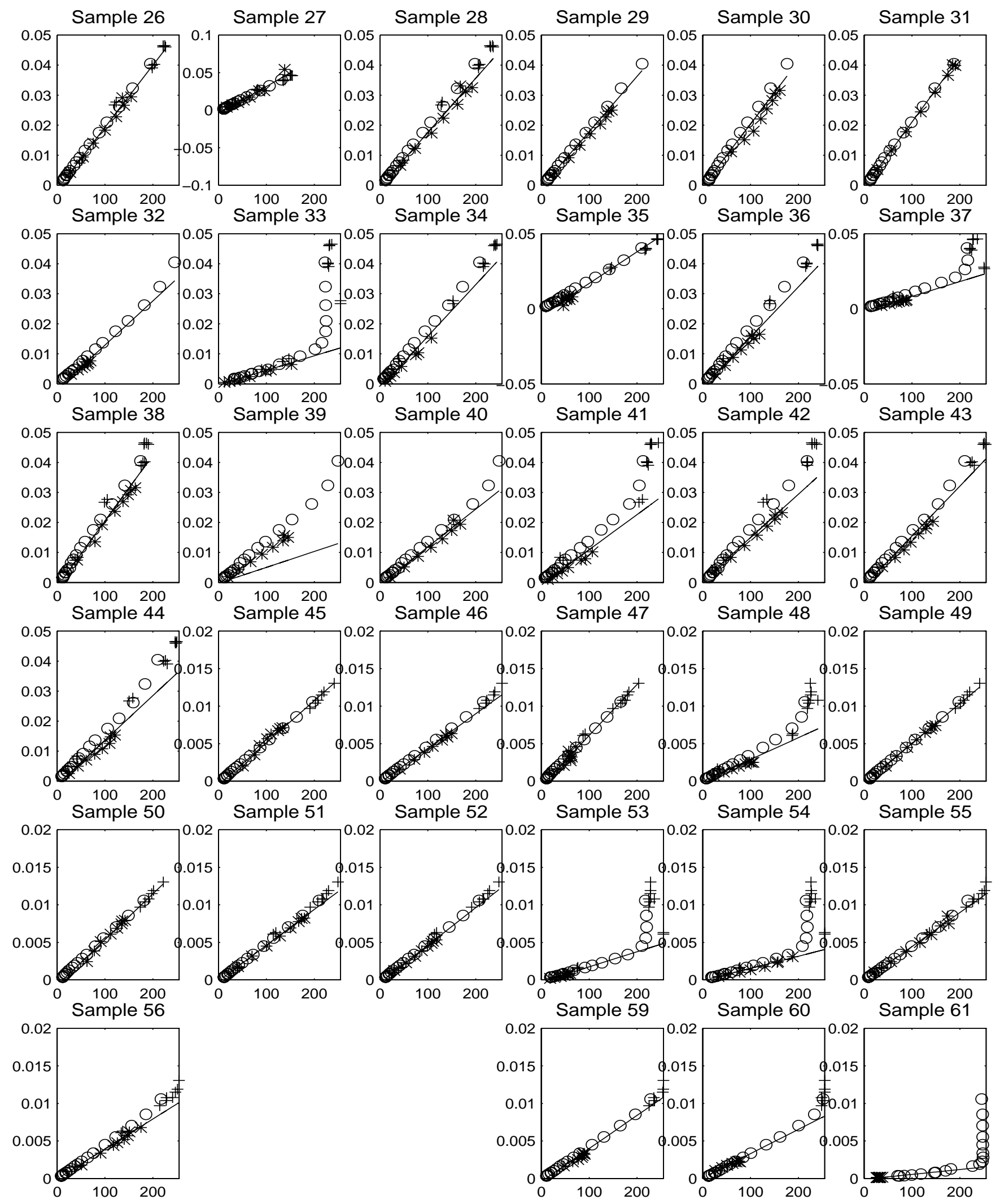

Figure 21: Calibration plots for the red calibration data for samples 26-61. The plotting symbols are described in Figure 18. 

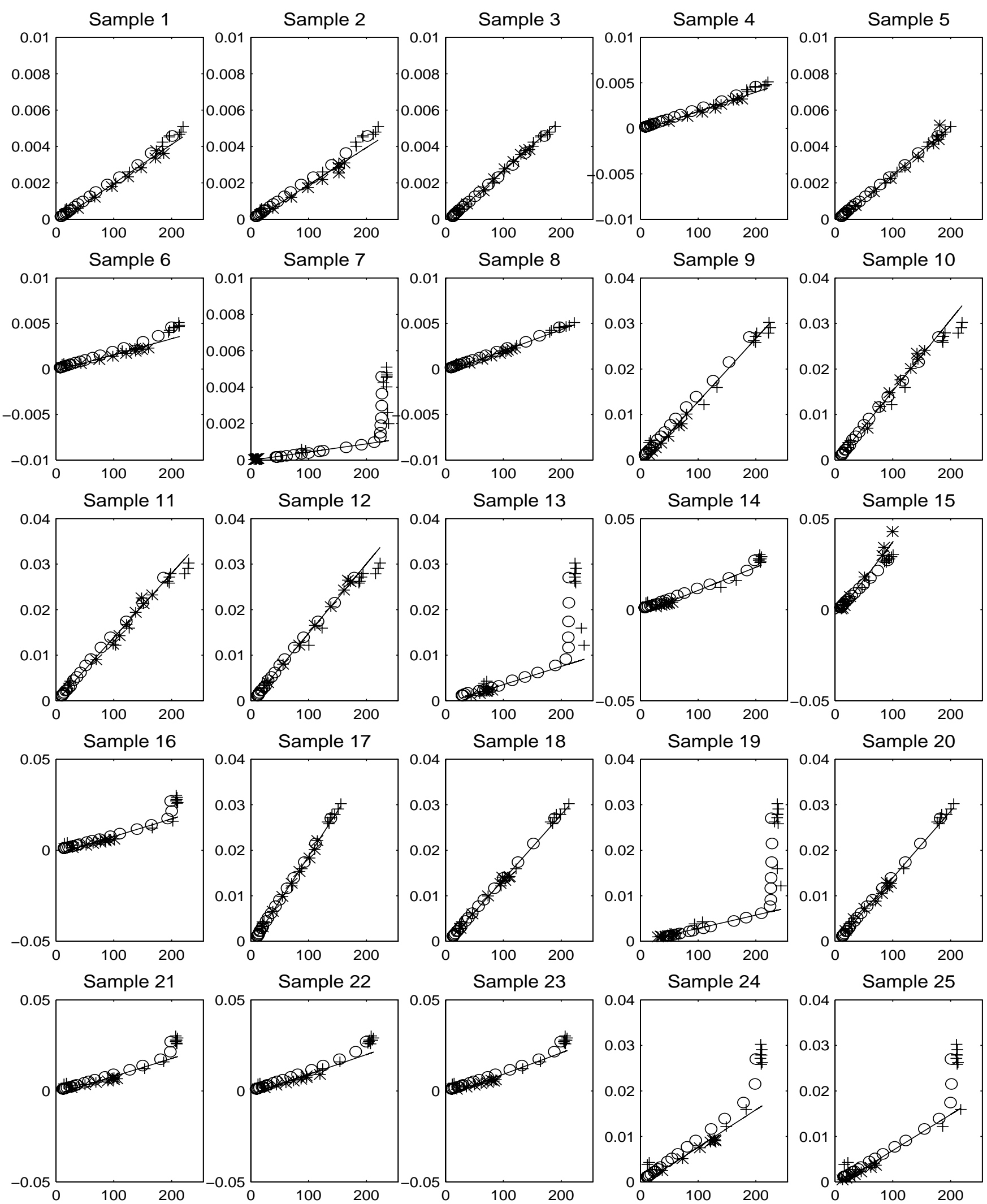

Figure 22: Calibration plots for the green calibration data for samples 1-25. The plotting symbols are described in Figure 18. 

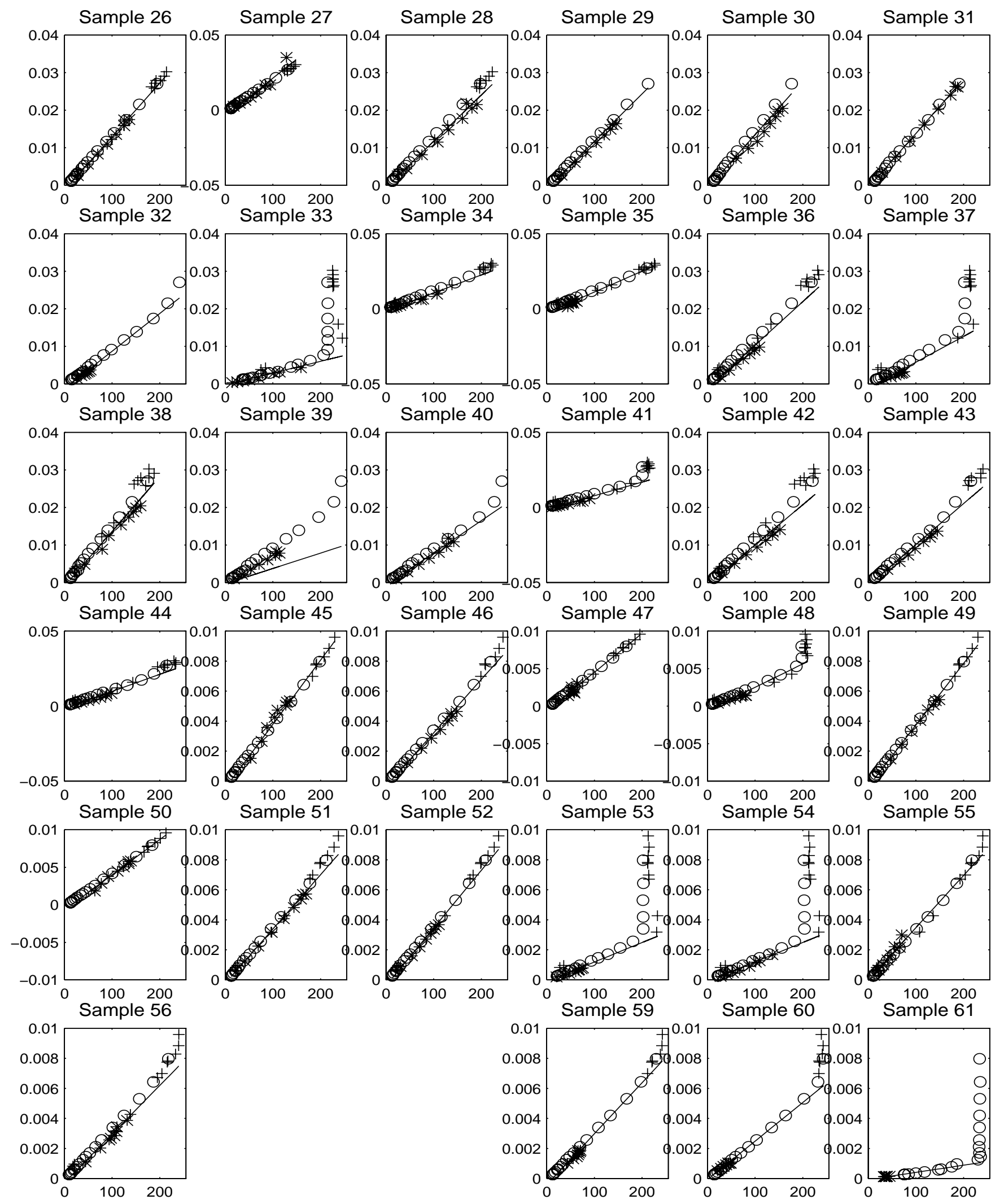

Figure 23: Calibration plots for the green calibration data for samples 26-61. The plotting symbols are described in Figure 18. 

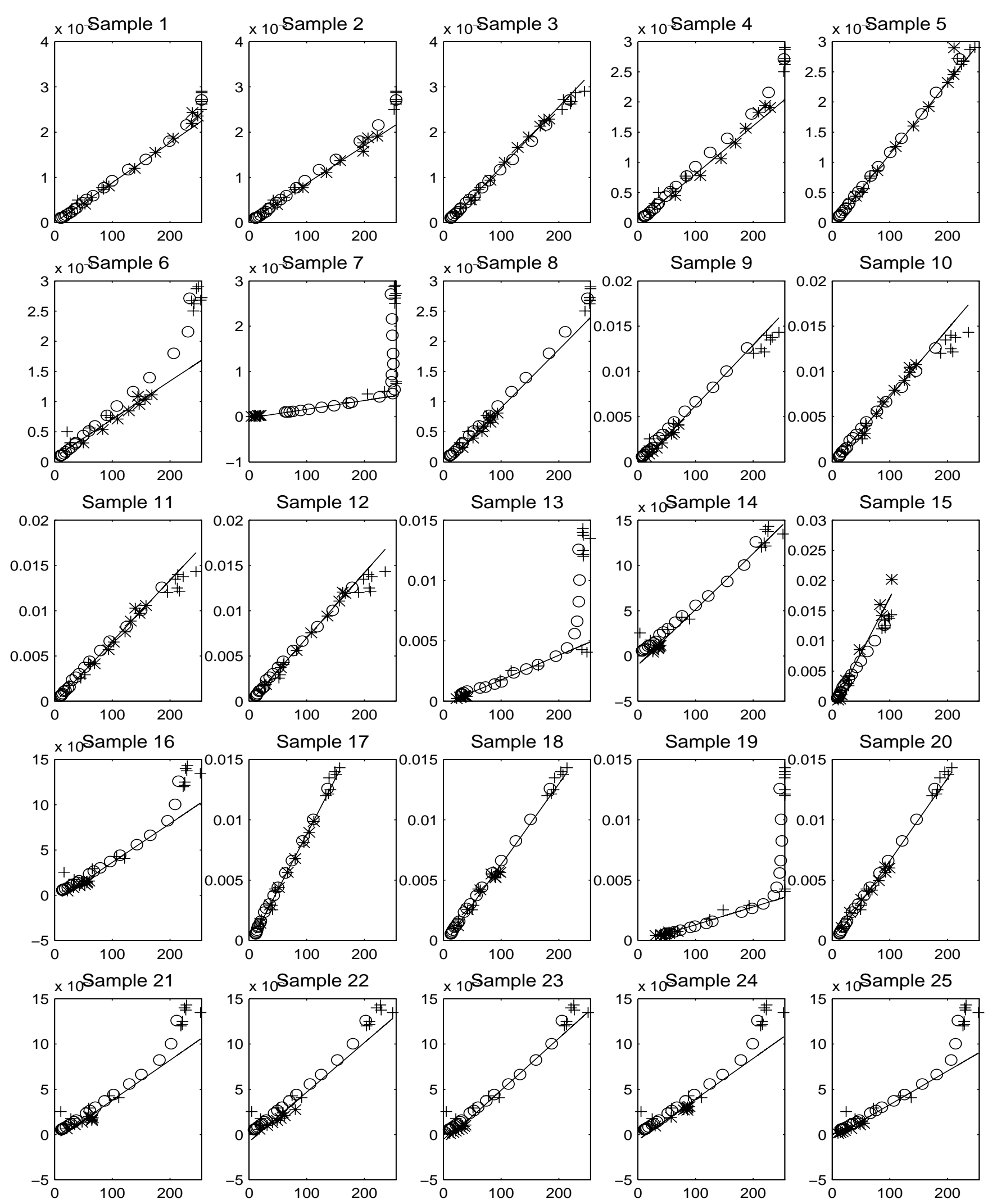

Figure 24: Calibration plots for the blue calibration data for samples 1-25. The plotting symbols are described in Figure 18. 

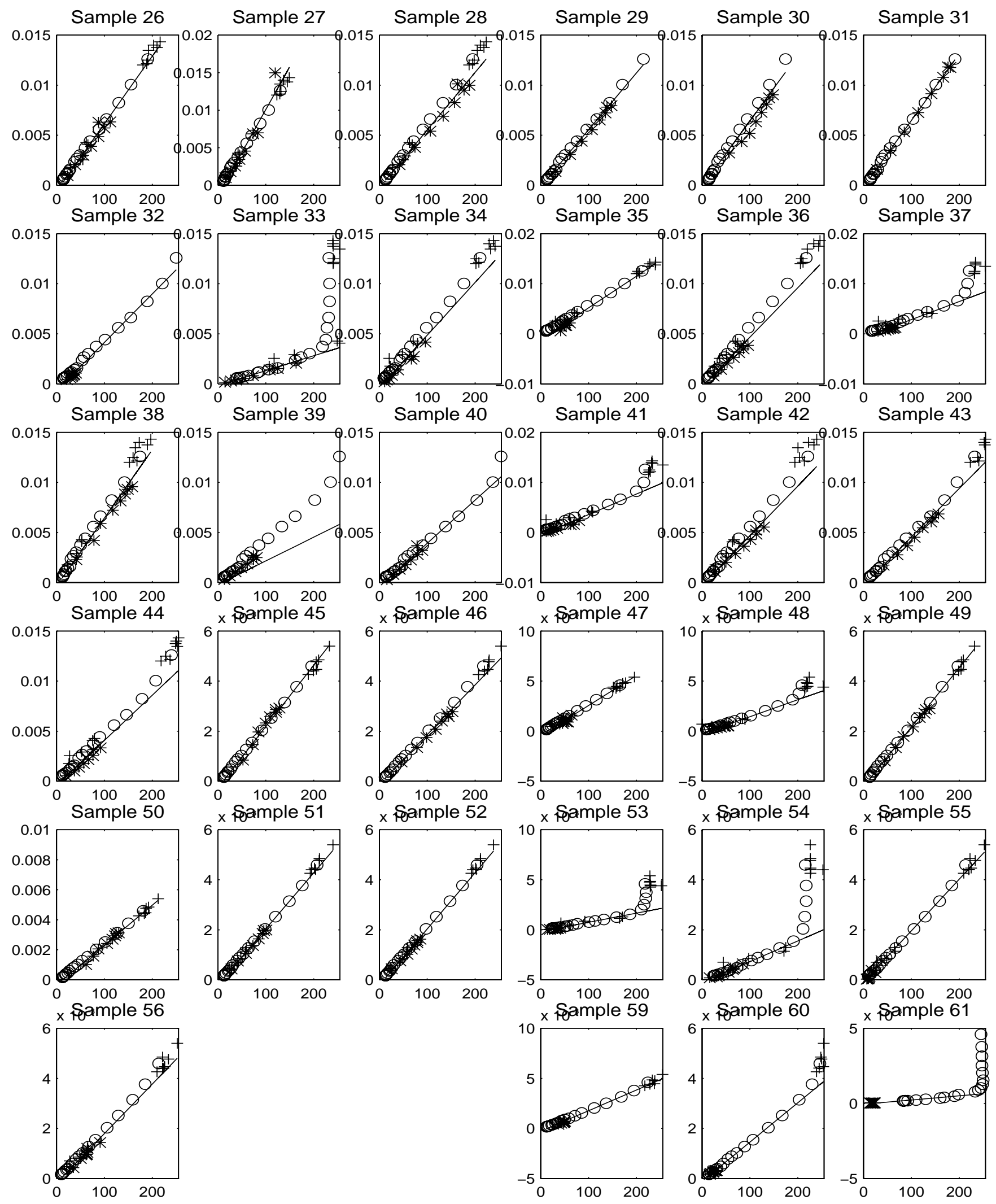

Figure 25: Calibration plots for the blue calibration data for samples 26-61. The plotting symbols are described in Figure 18. 


\section{FITTING RESULTS}

This section contains all the fitting results for the Oren-Nayar and Koenderink et al. model illustrated in Figures 26, 27, 28, 29, 30, 31, 32, 33, and 34. These figures have the same format as Figure 14. Note that Sample 57 is excluded because full segmentation of the peackock feather from the background is not possible. Also the glue used to adhere the feather contributes to the reflectance pattern. Sample 58 is not included because the sample was not globally flat. 

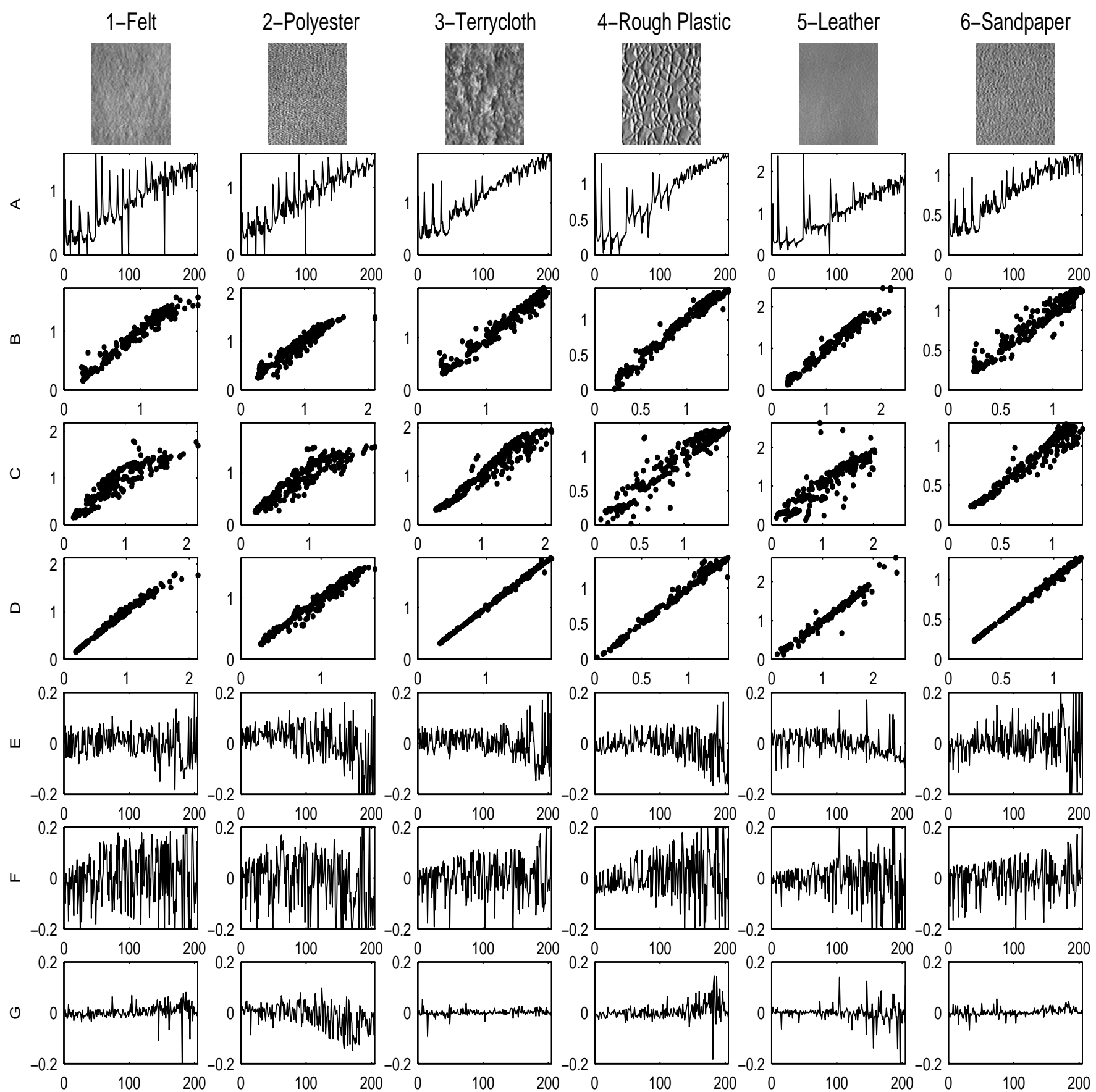

Figure 26: BRDF measurements and model-fitting results. Row A shows the raw measurement data plotted as a function of decreasing source angle to emphasize the non-Lambertian behavior of each sample. Rows B,C and D show the scatter-plots of the Oren-Nayar model fit (3 parameters), the order 2 Koenderink et al. fit ( 5 parameters) and the order 8 Koenderink et al. fit (55 parameters), respectively. Rows E,F and $\mathrm{G}$ show the residuals from the Oren-Nayar model fit, the order 2 Koenderink et al. fit and the order 8 Koenderink et al. fit, respectively. The residuals are plotted as a function of increasing viewing angle to show the concentration of errors at oblique views for some of the samples. The residuals shown are normalized by maximum measured radiance for that sample. 


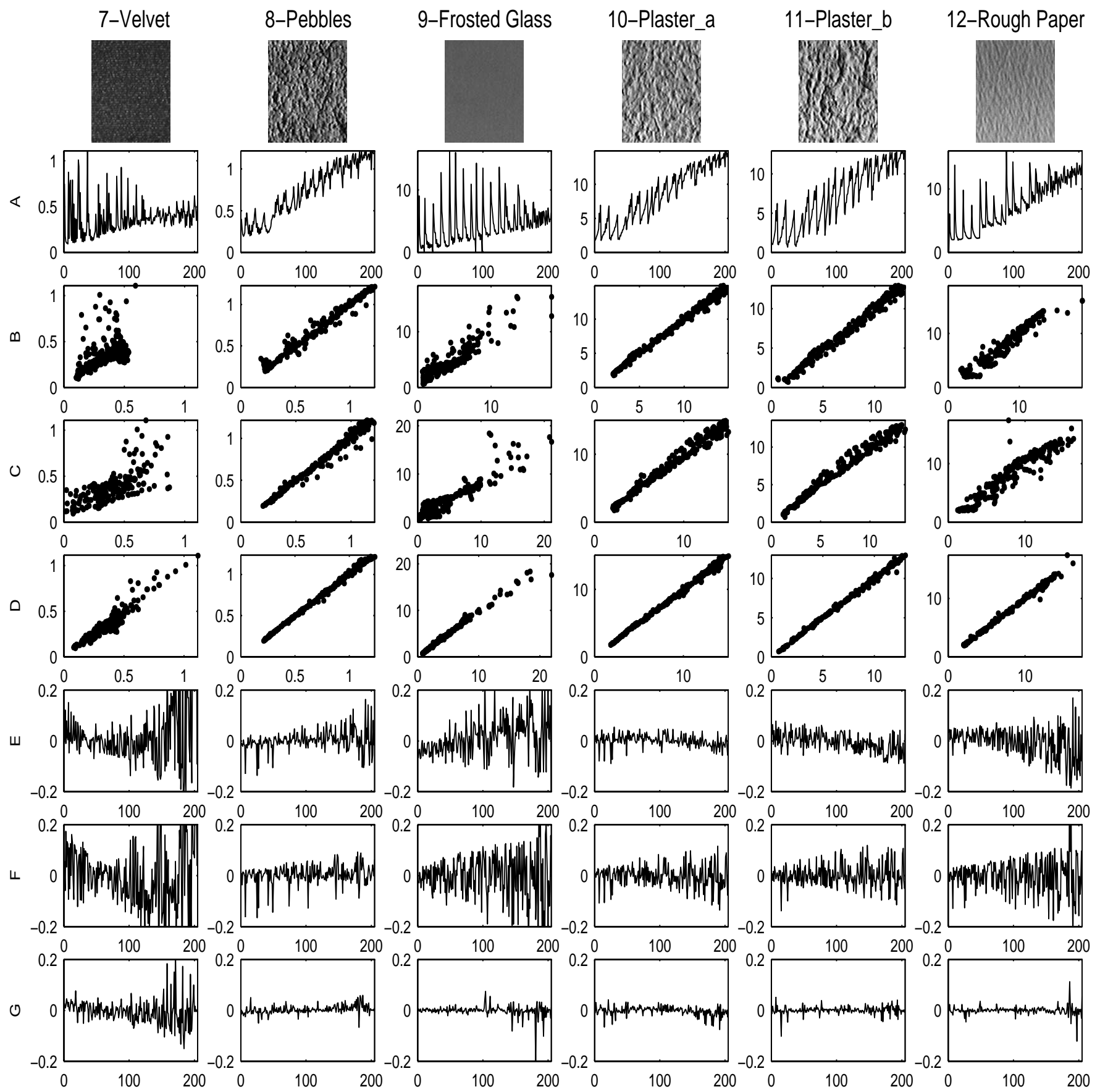

Figure 27: BRDF measurements and model-fitting results. Row A shows the raw measurement data plotted as a function of decreasing source angle to emphasize the non-Lambertian behavior of each sample. Rows B,C and D show the scatter-plots of the Oren-Nayar model fit (3 parameters), the order 2 Koenderink et al. fit ( 5 parameters) and the order 8 Koenderink et al. fit (55 parameters), respectively. Rows E,F and $\mathrm{G}$ show the residuals from the Oren-Nayar model fit, the order 2 Koenderink et al. fit and the order 8 Koenderink et al. fit, respectively. The residuals are plotted as a function of increasing viewing angle to show the concentration of errors at oblique views for some of the samples. The residuals shown are normalized by maximum measured radiance for that sample. 


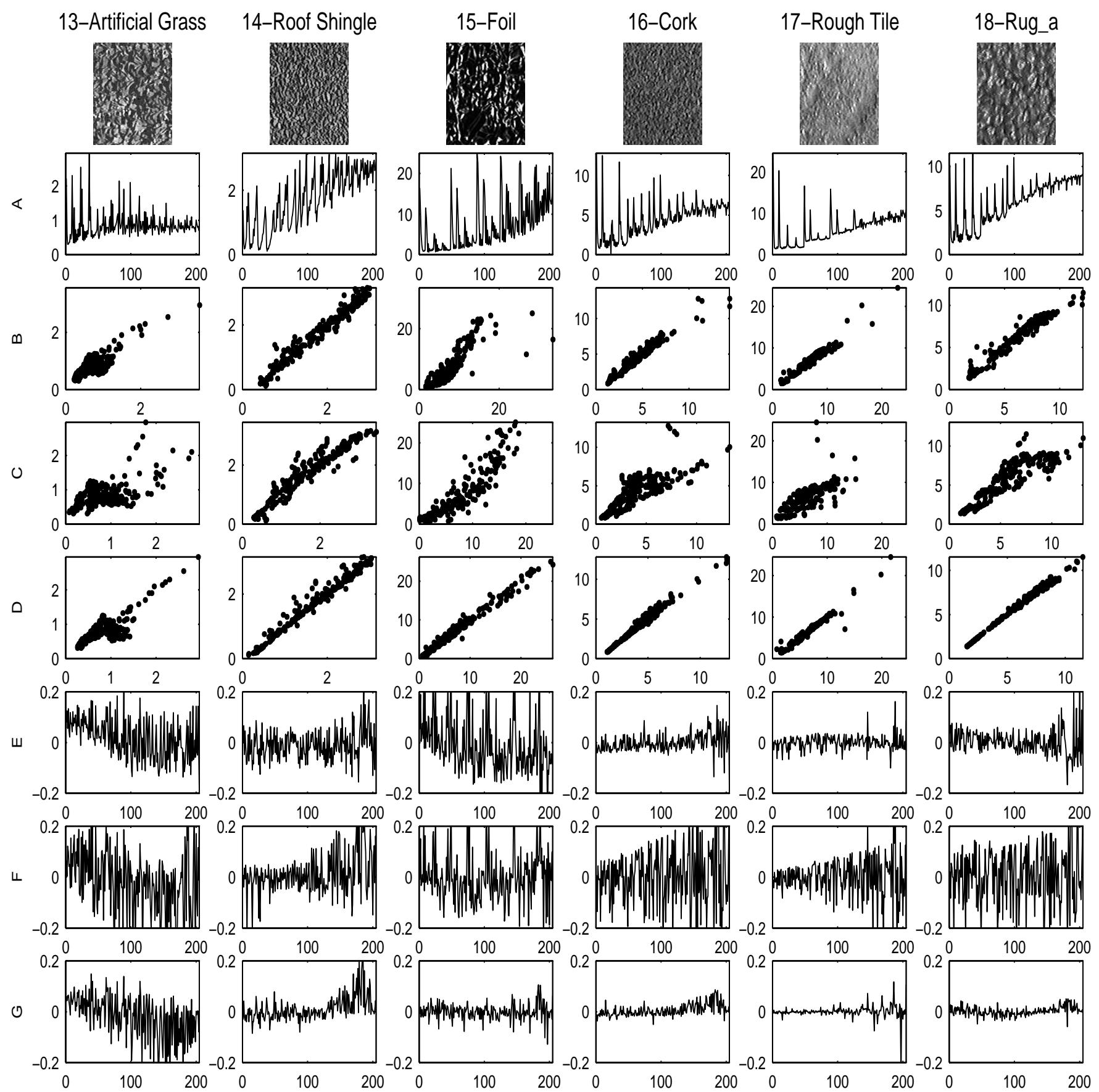

Figure 28: BRDF measurements and model-fitting results. Row A shows the raw measurement data plotted as a function of decreasing source angle to emphasize the non-Lambertian behavior of each sample. Rows B,C and D show the scatter-plots of the Oren-Nayar model fit (3 parameters), the order 2 Koenderink et al. fit ( 5 parameters) and the order 8 Koenderink et al. fit (55 parameters), respectively. Rows E,F and $\mathrm{G}$ show the residuals from the Oren-Nayar model fit, the order 2 Koenderink et al. fit and the order 8 Koenderink et al. fit, respectively. The residuals are plotted as a function of increasing viewing angle to show the concentration of errors at oblique views for some of the samples. The residuals shown are normalized by maximum measured radiance for that sample. 


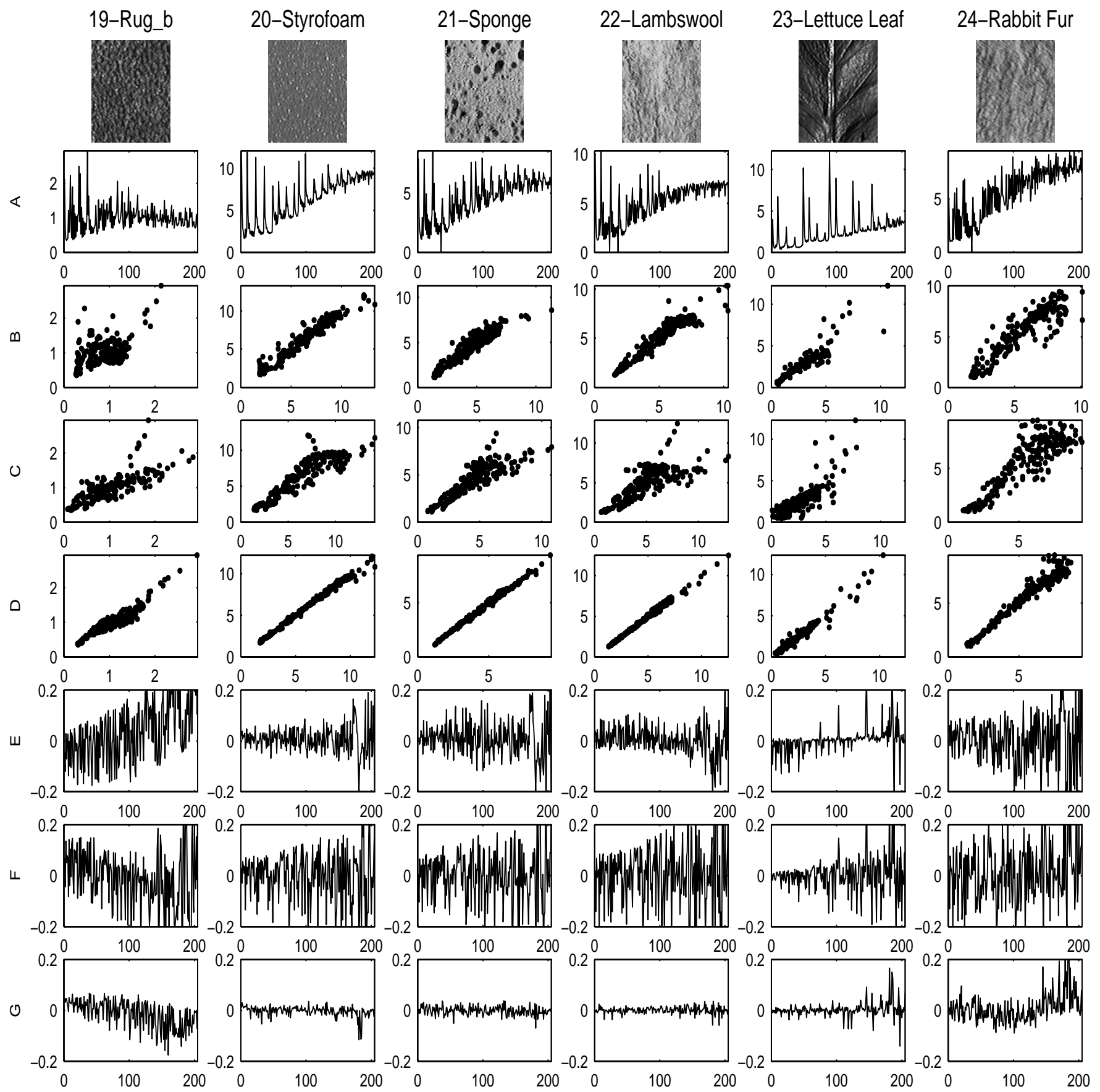

Figure 29: BRDF measurements and model-fitting results. Row A shows the raw measurement data plotted as a function of decreasing source angle to emphasize the non-Lambertian behavior of each sample. Rows B,C and D show the scatter-plots of the Oren-Nayar model fit (3 parameters), the order 2 Koenderink et al. fit ( 5 parameters) and the order 8 Koenderink et al. fit (55 parameters), respectively. Rows E,F and $\mathrm{G}$ show the residuals from the Oren-Nayar model fit, the order 2 Koenderink et al. fit and the order 8 Koenderink et al. fit, respectively. The residuals are plotted as a function of increasing viewing angle to show the concentration of errors at oblique views for some of the samples. The residuals shown are normalized by maximum measured radiance for that sample. 

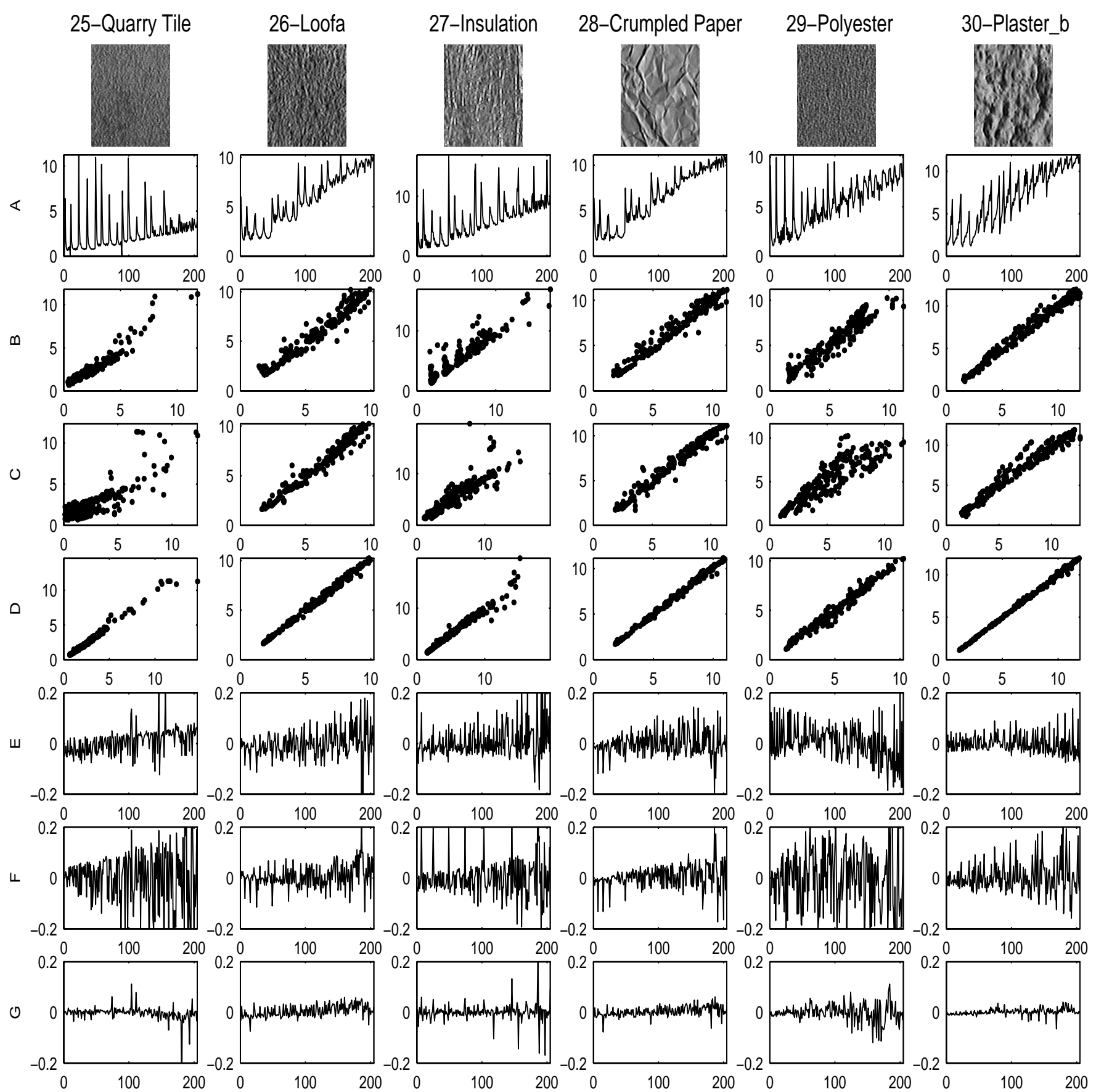

Figure 30: BRDF measurements and model-fitting results. Row A shows the raw measurement data plotted as a function of decreasing source angle to emphasize the non-Lambertian behavior of each sample. Rows B,C and D show the scatter-plots of the Oren-Nayar model fit (3 parameters), the order 2 Koenderink et al. fit ( 5 parameters) and the order 8 Koenderink et al. fit (55 parameters), respectively. Rows E,F and $\mathrm{G}$ show the residuals from the Oren-Nayar model fit, the order 2 Koenderink et al. fit and the order 8 Koenderink et al. fit, respectively. The residuals are plotted as a function of increasing viewing angle to show the concentration of errors at oblique views for some of the samples. The residuals shown are normalized by maximum measured radiance for that sample. 

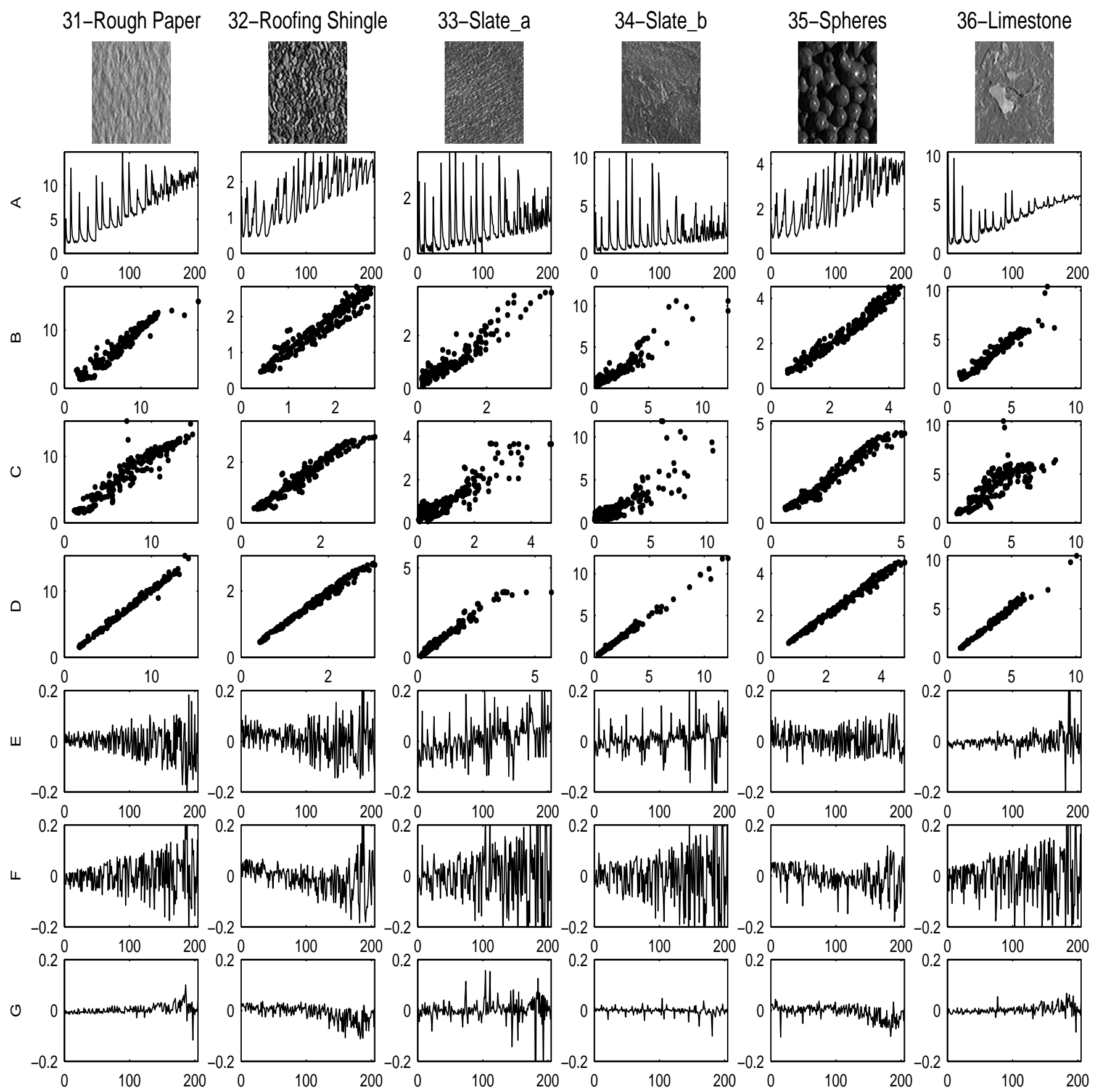

Figure 31: BRDF measurements and model-fitting results. Row A shows the raw measurement data plotted as a function of decreasing source angle to emphasize the non-Lambertian behavior of each sample. Rows B,C and D show the scatter-plots of the Oren-Nayar model fit (3 parameters), the order 2 Koenderink et al. fit ( 5 parameters) and the order 8 Koenderink et al. fit (55 parameters), respectively. Rows E,F and $\mathrm{G}$ show the residuals from the Oren-Nayar model fit, the order 2 Koenderink et al. fit and the order 8 Koenderink et al. fit, respectively. The residuals are plotted as a function of increasing viewing angle to show the concentration of errors at oblique views for some of the samples. The residuals shown are normalized by maximum measured radiance for that sample. 


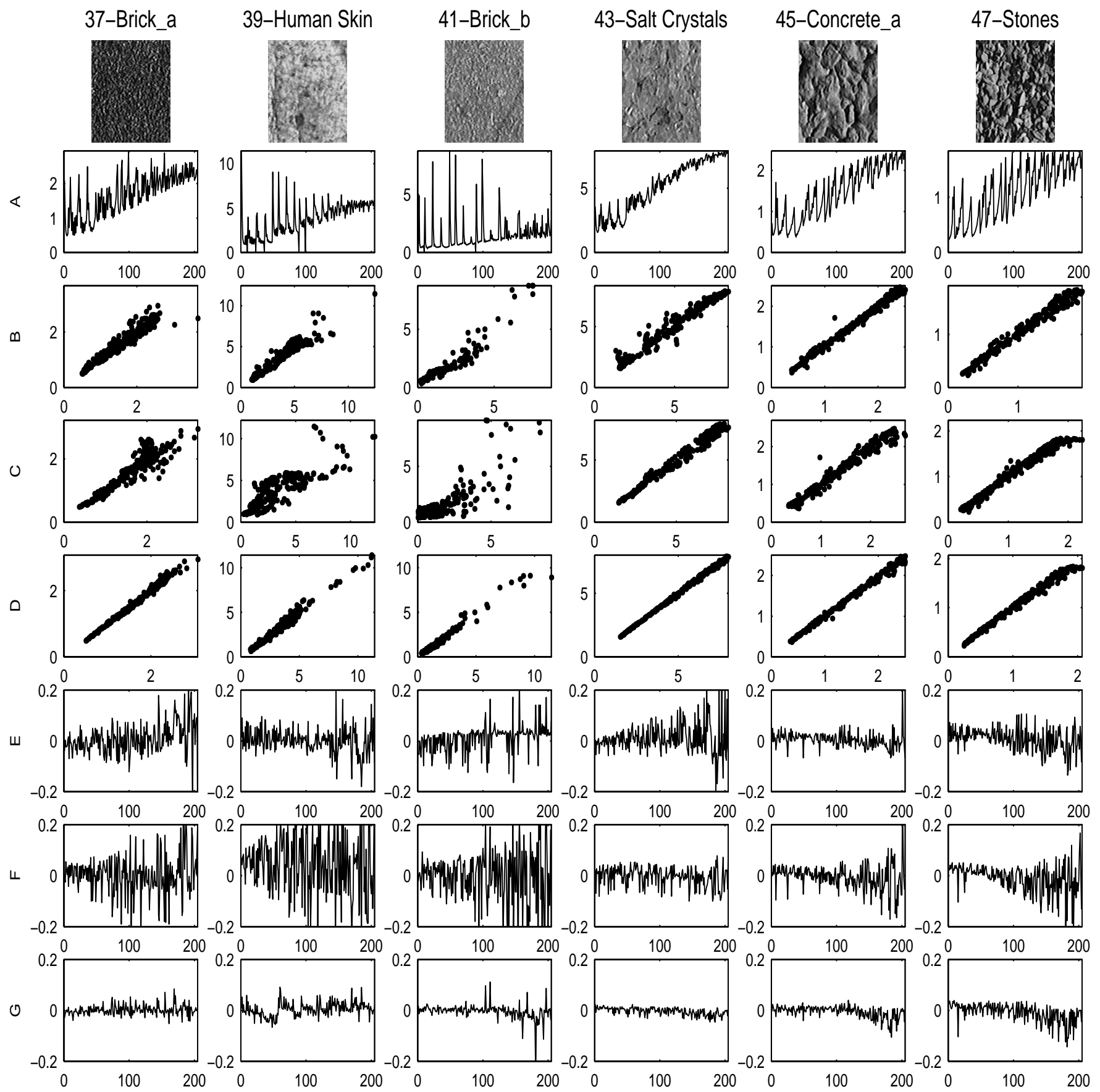

Figure 32: BRDF measurements and model-fitting results. Row A shows the raw measurement data plotted as a function of decreasing source angle to emphasize the non-Lambertian behavior of each sample. Rows B,C and D show the scatter-plots of the Oren-Nayar model fit (3 parameters), the order 2 Koenderink et al. fit ( 5 parameters) and the order 8 Koenderink et al. fit (55 parameters), respectively. Rows E,F and $\mathrm{G}$ show the residuals from the Oren-Nayar model fit, the order 2 Koenderink et al. fit and the order 8 Koenderink et al. fit, respectively. The residuals are plotted as a function of increasing viewing angle to show the concentration of errors at oblique views for some of the samples. The residuals shown are normalized by maximum measured radiance for that sample. 


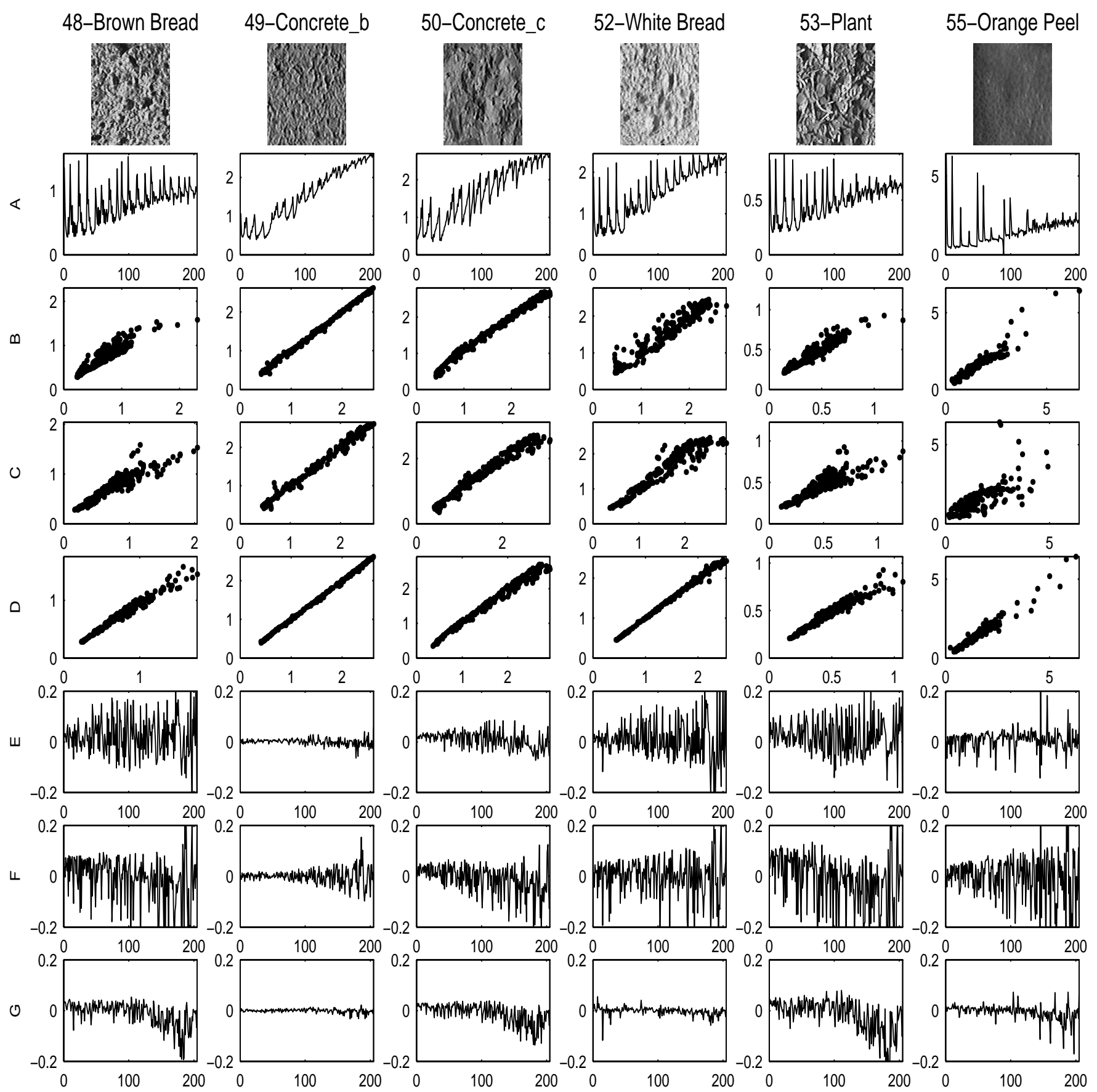

Figure 33: BRDF measurements and model-fitting results. Row A shows the raw measurement data plotted as a function of decreasing source angle to emphasize the non-Lambertian behavior of each sample. Rows B,C and D show the scatter-plots of the Oren-Nayar model fit (3 parameters), the order 2 Koenderink et al. fit ( 5 parameters) and the order 8 Koenderink et al. fit (55 parameters), respectively. Rows E,F and $\mathrm{G}$ show the residuals from the Oren-Nayar model fit, the order 2 Koenderink et al. fit and the order 8 Koenderink et al. fit, respectively. The residuals are plotted as a function of increasing viewing angle to show the concentration of errors at oblique views for some of the samples. The residuals shown are normalized by maximum measured radiance for that sample. 

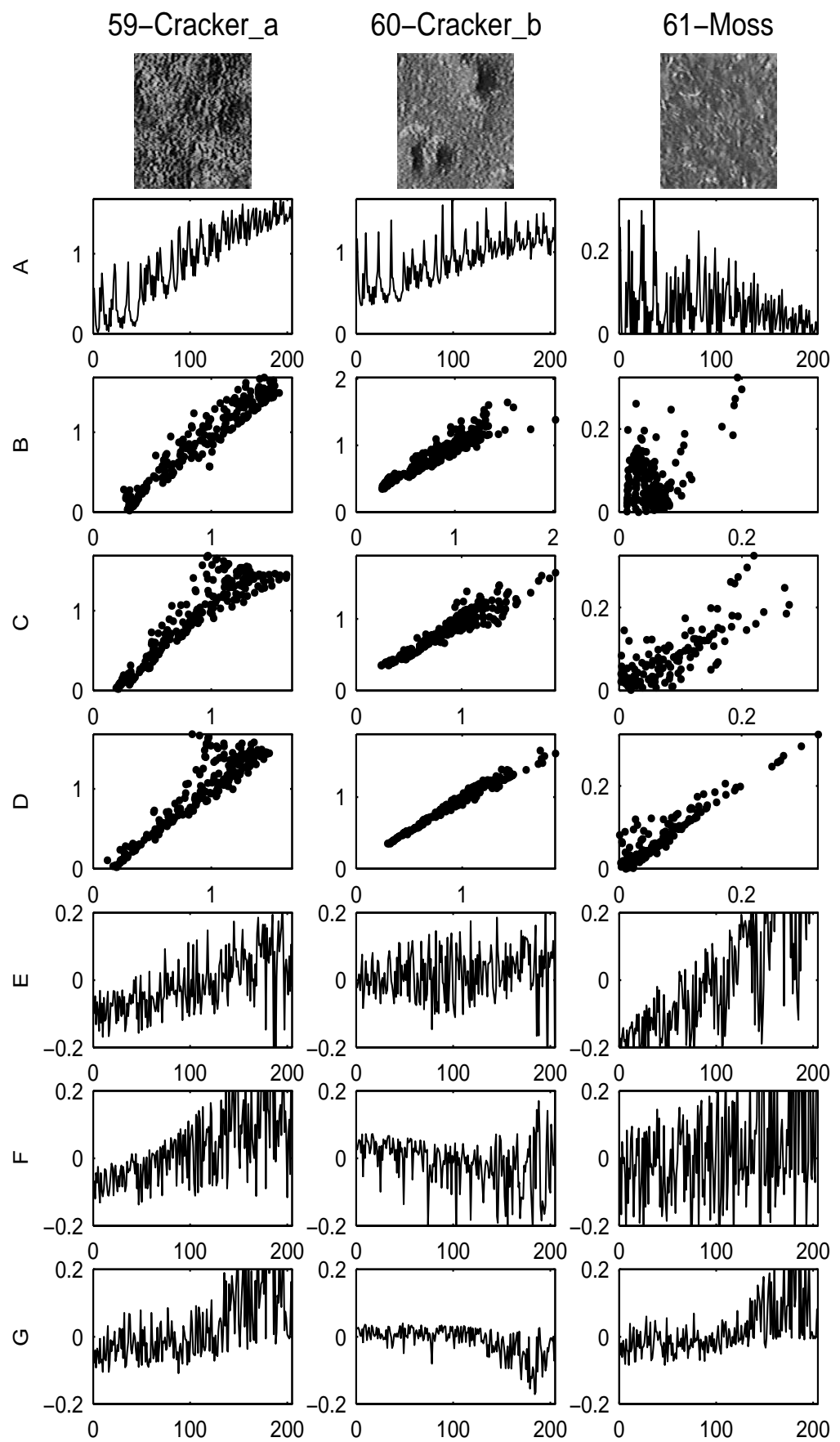

Figure 34: BRDF measurements and model-fitting results. Row A shows the raw measurement data plotted as a function of decreasing source angle to emphasize the non-Lambertian behavior of each sample. Rows B,C and D show the scatter-plots of the Oren-Nayar model fit (3 parameters), the order 2 Koenderink et al. fit ( 5 parameters) and the order 8 Koenderink et al. fit (55 parameters), respectively. Rows E,F and $\mathrm{G}$ show the residuals from the Oren-Nayar model fit, the order 2 Koenderink et al. fit and the order 8 Koenderink et al. fit, respectively. The residuals are plotted as a function of increasing viewing angle to show the concentration of errors at oblique views for some of the samples. The residuals shown are normalized by maximum measured radiance for that sample. 


\begin{tabular}{|c|l|l|l|l|}
\hline Lamp & Irradiance & $\sigma_{r}$ & $\sigma_{g}$ & $\sigma_{b}$ \\
\hline 1 & 9.56 & 0.0631 & 0.0620 & 0.0666 \\
2 & 57.664 & 0.0594 & 0.0605 & 0.0615 \\
3 & 13.0799 & 0.0496 & 0.0496 & 0.0529 \\
\hline
\end{tabular}

Table 10: Estimated irradiance and standard deviation of the normalized gray card image for each of the 3 lamps used in the measurements.

\section{E Light Sources}

Because of the limited lifetime of the lamps employed in the measurements, three different lamps were used:

- Lamp 1 was used for Samples 1-8.

- Lamp 2 was used for Samples 9-44.

- Lamp 3 was used for Samples 45-61.

Periodically during the measurements we perform a lamp calibration which consists of placing the photometer region of interest over each strip on the Kodak standard calibration card. This card consists of 20 grey strips (strip no. 0-19), a piece of a standard $18 \%$ grey card (strip no. 20) that was attached at the center of the card, and 12 color strips (strip no. 21-32). The purpose of these lamp calibrations is to relate pixel values to radiance values as described in Section C. (An image of the Kodak standard calibration card is obtained for each sample, after the camera aperture was set for that sample). The second purpose of the lamp calibrations is to determine the temporal stability of the lamp (whether it remained at approximately the same brightness for each day of the experiment)

For lamp 1, two calibrations were performed on Feb 2 and Feb 5 1996. Sample 1 was measured on Feb 6 and sample 8 was measured on Feb 5. Figure 35 shows the 32 photometer measurements from the standard calibration card for each of the lamp calibrations

For lamp 2, four calibrations were done on Feb 6,7,12 and 14. Sample 9 was measured on Feb 6 and sample 44 measured on Feb 15. Figure 36 shows the 32 photometer measurements from the standard calibration card for each of the lamp calibrations

For lamp 3 calibrations were done on Feb 19 and 23. Sample 45 was measured on Feb 16 and Sample 61 was measured on Feb 23. Figure 37 shows the 32 photometer measurements from the standard calibration card for each of the lamp calibrations

The plots from the 3 lamps show that spatial uniformity is reasonable and the lamps have good temporal stability.

Using the photometer measurements from the $18 \%$ gray card the irradiance was estimated. For lamps 1,2 and 3 the irradiance in watts $/ \mathrm{m}^{2}$ integrated over the wavelength range of $380 \mathrm{~nm}$ to $780 \mathrm{~nm}$ is shown in Table 10 .

The spatial uniformity of the lamps was analyzed by obtaining images of an 18of 22.5 degrees (i.e. with the camera in position 1) with the aperture set for the current sample. To summarize the result, we use one gray card image for each lamp (specifically we use the gray card images obtained when sample 8,25 and 47 were measured). The gray card images are normalized so that the maximum value is 1 and the standard deviation across the normalized card for each RGB channels $\left(\sigma_{r}, \sigma_{g}, \sigma_{b}\right)$ is computed. The results are shown in Table 10. 


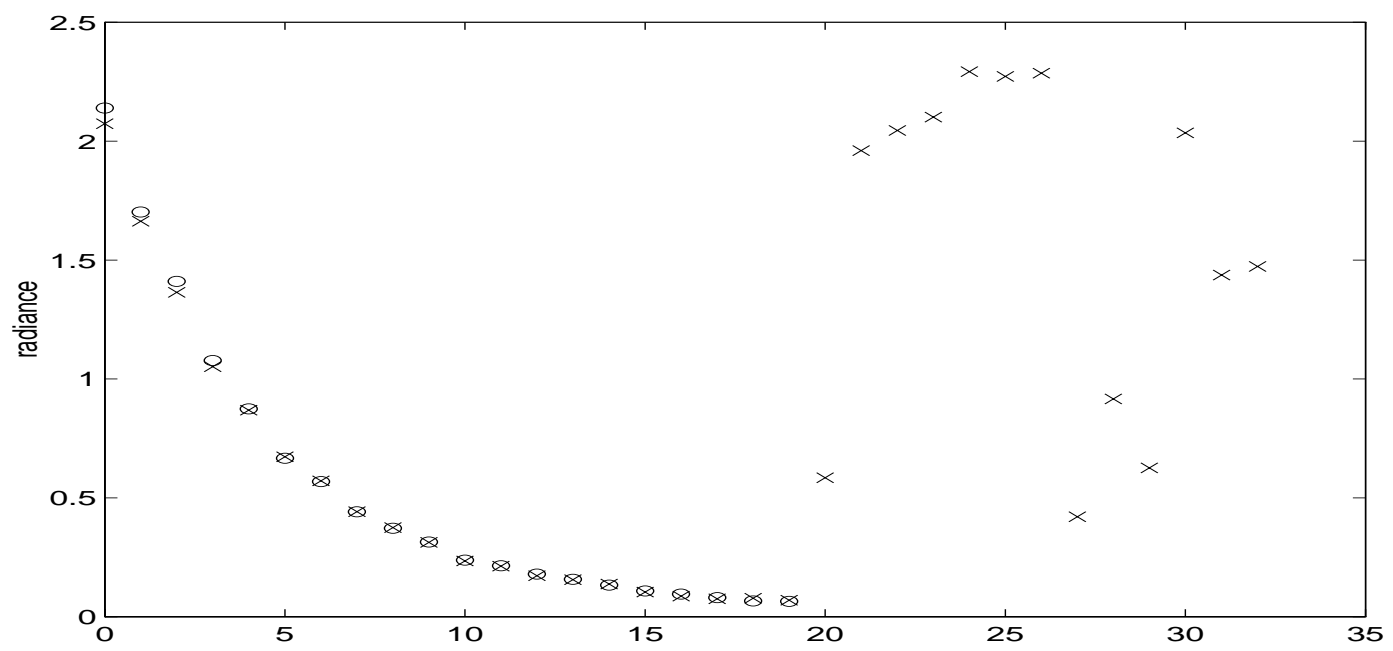

Figure 35: Lamp 1 calibration data. The first 20 points are the measured radiance from gray strips on the Kodak standard calibration card. The last 13 points are the measured radiance from the $18 \%$ gray card followed by the 12 color strips on the Kodak standard card. The lamp calibration was repeated twice on separate days. Data from the first set is plotted with the symbol 'o'. Data from the second set is plotted with the symbol ' $x$ '. (In this case the first calibration did not include the colored strips)

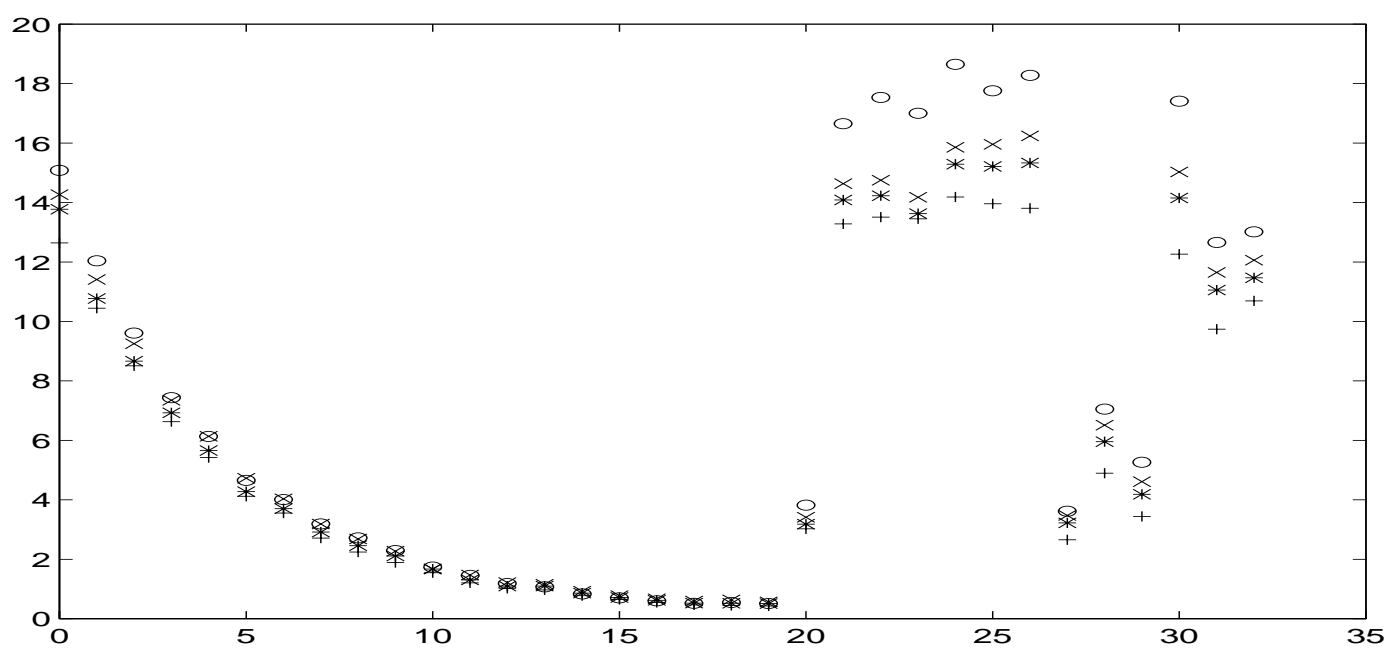

Figure 36: Lamp 2 calibration data. The first 20 points are the measured radiance from gray strips on the Kodak standard calibration card. The last 13 points are the measured radiance from the $18 \%$ gray card followed by the 12 color strips on the Kodak standard card. The lamp calibration was repeated four times on separate days. Data from the first, second, third and fourth calibrations are plotted with the symbols 'o', 'x', '*' and '+' respectively. Notice a small decrease in the lamp brightness with time. 


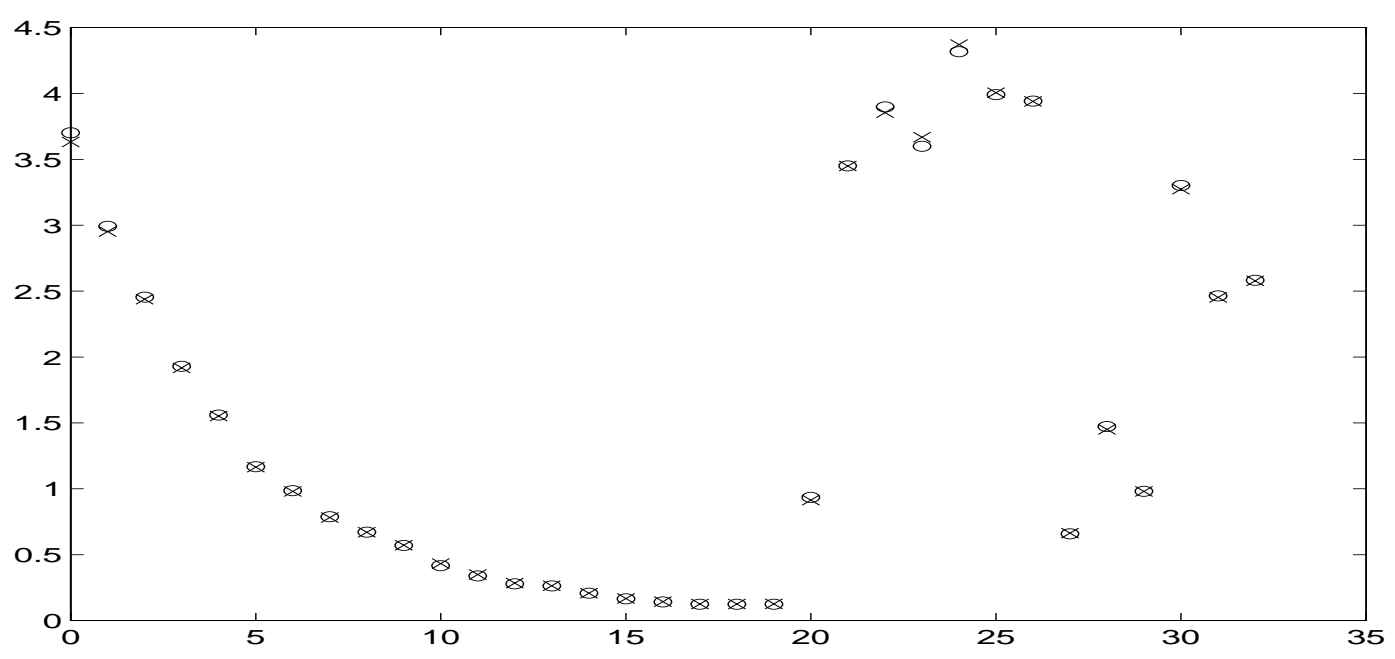

Figure 37: Lamp 3 calibration data. The first 20 points are the measured radiance from gray strips on the Kodak standard calibration card. The last 13 points are the measured radiance from the $18 \%$ gray card followed by the 12 color strips on the Kodak standard card. The lamp calibration was repeated twice on separate days. Data from the first set is plotted with the symbol 'o'. Data from the second set is plotted with the symbol ' $\mathrm{x}$ '.

\section{F Sources of Error}

A primary source of error in the experiments occured because of a brightness decrease that can be observed in the images when the robot edges specularly reflect the incident light. Although the camera's gain and gamma correction was turned off, a decrease in brightness of about 10was noticed in these specular positions. On average, this decrease affected 15 images of the 205 images obtained for each sample. A correction was devised by using the samples that were measured twice at two different magnifications. The zoomed view of these samples did not show the robot edges and provided ground truth to determine the exact magnitude of the brightness attenuation. By analyzing these images, it became clear that the empty image background around the sample provided a cue for when the brightness decrease occurred and the magnitude of that decrease. (By "empty background" we mean there was no significant reflection since the only object was a relatively distant wall.) This relation between background pixel value and intensity dip was independent of the aperture and light source since it depends only on the dark current of the camera. The correction procedure is as follows: 1) manually segment the background in the specular and near specular images to obtain an average background value $B, 2$ ) if the background pixel value is less than 7 apply divide the image by a correction factor of $0.0360 B+0.7236$. (This number was obtained by using the ground truth provided by the measurements of Samples 2,11,12,14 under two different magnifications. Under large magnification the robot edges were not visible and therefore the intensity decrease did not occur).

Tables 11 and 12 have a list of the images that were corrected with the corresponding correction factor (applied to $\mathrm{R} / \mathrm{G} / \mathrm{B}$ ). The image number provided in these tables is the same as that shown in the tables of Section A. 


\begin{tabular}{|c|c|c|c|}
\hline \multicolumn{2}{|c|}{ Sample 1} & \multicolumn{2}{|c|}{ Sample 4 (contd.) } \\
\hline 45 & 1.079373 & 129 & 1.112792 \\
\hline 48 & 1.101738 & 131 & 1.144691 \\
\hline 49 & 1.061542 & 133 & 1.077222 \\
\hline 51 & 1.175437 & 134 & 1.128846 \\
\hline 52 & 1.040517 & 136 & 1.118431 \\
\hline 53 & 1.124913 & 138 & 1.111562 \\
\hline 54 & 1.147310 & 140 & 1.053150 \\
\hline 55 & 1.115049 & 141 & 1.127432 \\
\hline 94 & 1.178668 & 142 & 1.077667 \\
\hline 97 & 1.170437 & 144 & 1.137630 \\
\hline 102 & 1.033554 & 150 & 1.085091 \\
\hline 128 & 1.133726 & 152 & 1.103637 \\
\hline 131 & 1.085486 & 156 & 1.118610 \\
\hline 144 & 1.117670 & 158 & 1.125850 \\
\hline 160 & 1.070285 & 160 & 1.122924 \\
\hline 183 & 1.152631 & 161 & 1.117311 \\
\hline 184 & 1.122306 & 163 & 1.118738 \\
\hline 192 & 1.026198 & 164 & 1.084392 \\
\hline 196 & 1.193183 & 165 & 1.105069 \\
\hline 197 & 1.185807 & 167 & 1.107965 \\
\hline 202 & 1.176015 & 168 & 1.124916 \\
\hline 203 & 1.178312 & 170 & 1.028305 \\
\hline 204 & 1.181993 & 171 & 1.069746 \\
\hline 205 & 1.161660 & 173 & 1.072757 \\
\hline \multicolumn{2}{|c|}{ Sample 2} & 178 & 1.131755 \\
\hline 51 & 1.126835 & 179 & 1.137829 \\
\hline 94 & 1.110561 & 181 & 1.119112 \\
\hline 97 & 1.106727 & 183 & 1.157139 \\
\hline 128 & 1.083106 & 184 & 1.149225 \\
\hline 131 & 1.137244 & 186 & 1.107010 \\
\hline 144 & 1.117708 & 187 & 1.143238 \\
\hline 183 & 1.162915 & 188 & 1.113137 \\
\hline 184 & 1.070157 & 189 & 1.124941 \\
\hline 196 & 1.156278 & 190 & 1.150286 \\
\hline 197 & 1.117207 & 191 & 1.139191 \\
\hline 202 & 1.198176 & 193 & 1.132960 \\
\hline 203 & 1.188463 & 196 & 1.182219 \\
\hline 204 & 1.181683 & 197 & 1.184758 \\
\hline 205 & 1.151726 & 198 & 1.153263 \\
\hline \multicolumn{2}{|c|}{ Sample 3} & 200 & 1.185897 \\
\hline 51 & 1.129194 & 201 & 1.170269 \\
\hline 94 & 1.064870 & 202 & 1.214484 \\
\hline 97 & 1.093310 & 203 & 1.200676 \\
\hline 128 & 1.072703 & 204 & 1.186258 \\
\hline 131 & 1.140562 & 205 & 1.077818 \\
\hline 144 & 1.082407 & & nple 5 \\
\hline 183 & 1.152882 & 45 & 1.045033 \\
\hline 196 & 1.153164 & 51 & 1.127345 \\
\hline 202 & 1.145036 & 54 & 1.147207 \\
\hline 203 & 1.115905 & 97 & 1.134828 \\
\hline & mple 4 & 128 & 1.073797 \\
\hline 37 & 1.076511 & 131 & 1.148850 \\
\hline 39 & 1.101676 & 144 & 1.093714 \\
\hline 42 & 1.087668 & 161 & 1.126711 \\
\hline 43 & 1.061210 & 183 & 1.153973 \\
\hline 44 & 1.065653 & 196 & 1.175482 \\
\hline 45 & 1.133849 & 197 & 1.123705 \\
\hline 46 & 1.130772 & 202 & 1.139320 \\
\hline 47 & 1.093994 & 203 & 1.185002 \\
\hline 48 & 1.111099 & & mple 6 \\
\hline 49 & 1.102353 & 45 & 1.082838 \\
\hline 50 & 1.110126 & 49 & 1.082399 \\
\hline 51 & 1.144965 & 51 & 1.134153 \\
\hline 52 & 1.127164 & 54 & 1.131921 \\
\hline 53 & 1.120129 & 55 & 1.087730 \\
\hline 54 & 1.116050 & 90 & 1.073045 \\
\hline 55 & 1.115422 & 94 & 1.134479 \\
\hline 81 & 1.078478 & 97 & 1.137385 \\
\hline 85 & 1.134638 & 102 & 1.081642 \\
\hline 87 & 1.117611 & 125 & 1.066124 \\
\hline 90 & 1.117678 & 128 & 1.127388 \\
\hline 93 & 1.103686 & 131 & 1.130007 \\
\hline 94 & 1.119205 & 136 & 1.081905 \\
\hline 96 & 1.076898 & 144 & 1.142828 \\
\hline 97 & 1.142146 & 160 & 1.091740 \\
\hline 98 & 1.066414 & 183 & 1.150291 \\
\hline 99 & 1.114409 & 184 & 1.087037 \\
\hline 100 & 1.087192 & 192 & 1.036779 \\
\hline 101 & 1.121016 & 197 & 1.044243 \\
\hline 102 & 1.107502 & 202 & 1.154921 \\
\hline 103 & 1.128022 & 203 & 1.140791 \\
\hline 119 & 1.096861 & 204 & 1.087876 \\
\hline 121 & 1.117565 & & \\
\hline 123 & 1.064564 & & \\
\hline 125 & 1.120929 & & \\
\hline 128 & 1.147033 & & \\
\hline
\end{tabular}

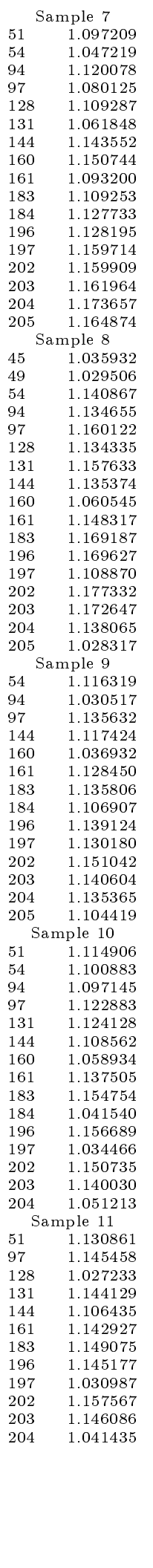

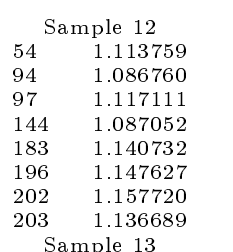

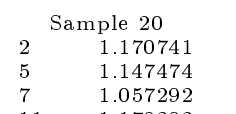

1.178292

1.053249
1.053635

1.053635
1.160292

1.040716

1.058004

1.089831
1.109915

1.056048

1.125099

1.053035
1.184188

1.066100
1.172585

1.172585

1.104929

1.090404

1.127859
1.118355

1.118355
1.085025

1.104446

1.154265

1.123192

1.146665

1.101637
1.157244

1.157244
1.134012

1.139748

1.132234

1.148994
1.128686
1.131146

1.131146

1.131146
1.081622

1.081622
1.080721

1.151251

1.149980
1.140966

$\begin{array}{ll}204 & 1.140966 \\ 205 & 1.131308\end{array}$

$\begin{array}{lr} & \text { Sample } 21 \\ 1 & 1.053210 \\ 2 & 1.171231 \\ 5 & 1.162381\end{array}$

1.162381

1.102724
1.172192

1.134451

1.121852

1.149665

1.142746

1.142746
1.135820

1.087658

1.104530

1.130071
1.051123

1.114552

1.103577

1.125898

1.043631

1.125300

1.124806

1.086745
1.088366
1.076061

1.088366
1.076061

1.110757

1.109612

1.071645

1.120705
1.109649

1.109649
1.108167

1.126907

1.175180

1.157649
1.149917

1.152066

1.152066
1.106213

1.106213
1.106717

1.139506

1.073866

1.048171

1.139771
1.041944

1.041944
1.133446

1.108618
Sample 21 (contd.)

$95 \quad 1.123563$

$97-1.108552$

$100 \quad 1.122903$

$101 \quad 1.080965$

$\begin{array}{ll}102 & 1.091687 \\ 103 & 1.103451\end{array}$

$104 \quad 1.096812$

$105 \quad 1.082452$

$106 \quad 1.050722$

$\begin{array}{ll}112 & 1.049458 \\ 113 & 1.105664\end{array}$

$115 \quad 1.105578$

$\begin{array}{ll}122 & 1.128889 \\ 123 & 1.102199\end{array}$

$125 \quad 1.110371$

$\begin{array}{ll}128 & 1.111139\end{array}$

$131 \quad 1.100785$

$\begin{array}{ll}137 & 1.119677\end{array}$

$\begin{array}{ll}138 & 1.074962 \\ 139 & 1.133332\end{array}$

$140 \quad 1.112010$

$141 \quad 1.112979$

$\begin{array}{ll}142 & 1.123912 \\ 144 & 1.139387\end{array}$

$\begin{array}{ll}144 & 1.139387 \\ 153 & 1.122374\end{array}$

$153 \quad 1.129291$

$\begin{array}{ll}155 & 1.074760 \\ 160 & 1.115803\end{array}$

$\begin{array}{ll}161 & 1.096068\end{array}$

$\begin{array}{ll}165 & 1.067887 \\ 167 & 1.043697\end{array}$

$168 \quad 1.063632$

$\begin{array}{ll}169 & 1.085290\end{array}$

$\begin{array}{ll}170 & 1.104357\end{array}$

$\begin{array}{ll}174 & 1.120648 \\ 176 & 1.040611\end{array}$

$178 \quad 1.105713$

$\begin{array}{ll}181 & 1.094553 \\ 182 & 1.130670\end{array}$

$183 \quad 1.133106$

$184 \quad 1.134645$

$187 \quad 1.128563$

$188 \quad 1.059383$

$\begin{array}{ll}189 & 1.082745 \\ 192 & 1.119753\end{array}$

$194 \quad 1.141635$

$195 \quad 1.118915$

$196 \quad 1.131709$

$198 \quad 1.099317$

$199 \quad 1.083855$

$\begin{array}{ll}200 & 1.137635 \\ 201 & 1.125835\end{array}$

$202 \quad 1.157410$

$203 \quad 1.151710$

$205 \quad 1.153792$

Sample 22

$49 \begin{array}{rr}\text { Sample } 22 \\ 51 & 1.051689 \\ 55 & 1.115562\end{array}$

$\begin{array}{ll}51 & 1.115562 \\ 55 & 1.075901\end{array}$

$54 \quad 1.106653$

$\begin{array}{ll}54 & 1.107006\end{array}$

$\begin{array}{ll}94 & 1.115597 \\ 97 & 1.108659\end{array}$

$128 \quad 1.096970$

$131 \quad 1.101988$

$144 \quad 1.166856$

$\begin{array}{ll}161 & 1.124724 \\ 183 & 1.150621\end{array}$

$184 \quad 1.121190$

$\begin{array}{ll}192 & 1.079414 \\ 192 & 1.078321\end{array}$

$196 \quad 1.137415$

$197 \quad 1.128607$

$202 \quad 1.154876$

$03 \quad 1.144825$

$\begin{array}{ll}204 & 1.158918 \\ 205 & 1.153635\end{array}$

Table 11: Correction factors for each sample. The left column of each table has the image number and the right column has the correction factor. 


\begin{tabular}{|c|c|c|c|}
\hline & pple 23 & & aple 28 \\
\hline 45 & 1.088045 & 51 & 1.114429 \\
\hline 49 & 1.128477 & 54 & 1.108953 \\
\hline 51 & 1.118224 & 97 & 1.120979 \\
\hline 55 & 1.119940 & 131 & 1.116225 \\
\hline 54 & 1.111257 & 144 & 1.135485 \\
\hline 94 & 1.121834 & 160 & 1.070874 \\
\hline 94 & 1.122777 & 161 & 1.133031 \\
\hline 97 & 1.112560 & 183 & 1.142224 \\
\hline 102 & 1.059114 & 184 & 1.081898 \\
\hline 125 & 1.069168 & 196 & 1.142109 \\
\hline 128 & 1.118232 & 197 & 1.122862 \\
\hline 131 & 1.121171 & 202 & 1.153888 \\
\hline 136 & 1.118206 & 203 & 1.132397 \\
\hline 144 & 1.162420 & 204 & 1.119404 \\
\hline 160 & 1.119135 & & ple 29 \\
\hline 161 & 1.096005 & 202 & 1.133970 \\
\hline 183 & 1.136331 & 203 & 1.123710 \\
\hline 184 & 1.144806 & 204 & 1.137990 \\
\hline 184 & 1.138708 & 205 & 1.117786 \\
\hline 192 & 1.067843 & 144 & 1.164473 \\
\hline 196 & 1.148243 & & ple 30 \\
\hline 197 & 1.148511 & 144 & 1.166937 \\
\hline 202 & 1.152126 & & ple 31 \\
\hline 203 & 1.141178 & 202 & 1.161739 \\
\hline 204 & 1.134701 & 203 & 1.101205 \\
\hline 205 & 1.069701 & 144 & 1.168618 \\
\hline & aple 24 & & pple 32 \\
\hline 51 & 1.093023 & 144 & 1.174087 \\
\hline 54 & 1.050768 & & ple 33 \\
\hline 94 & 1.099084 & 51 & 1.101791 \\
\hline 97 & 1.085464 & 54 & 1.032469 \\
\hline 128 & 1.114114 & 94 & 1.127876 \\
\hline 144 & 1.141887 & 97 & 1.060821 \\
\hline 160 & 1.091848 & 128 & 1.131260 \\
\hline 161 & 1.105780 & 131 & 1.086977 \\
\hline 183 & 1.135030 & 160 & 1.144125 \\
\hline 184 & 1.129164 & 161 & 1.039345 \\
\hline 196 & 1.144034 & 183 & 1.065172 \\
\hline 197 & 1.139857 & 184 & 1.115384 \\
\hline 202 & 1.138149 & 196 & 1.096457 \\
\hline 203 & 1.125823 & 197 & 1.139214 \\
\hline 204 & 1.136848 & 202 & 1.050818 \\
\hline 205 & 1.139288 & 204 & 1.156875 \\
\hline & pple 25 & 205 & 1.145880 \\
\hline 45 & 1.074921 & 144 & 1.131027 \\
\hline 51 & 1.122910 & & ple 34 \\
\hline 54 & 1.052832 & 51 & 1.120682 \\
\hline 94 & 1.126763 & 183 & 1.133618 \\
\hline 97 & 1.126482 & 184 & 1.098476 \\
\hline 128 & 1.127354 & 196 & 1.146216 \\
\hline 131 & 1.114814 & 197 & 1.140753 \\
\hline 144 & 1.147864 & 202 & 1.142840 \\
\hline 160 & 1.130750 & 203 & 1.131744 \\
\hline 183 & 1.115710 & 204 & 1.143481 \\
\hline 184 & 1.130634 & 205 & 1.029775 \\
\hline 192 & 1.088329 & 144 & 1.122662 \\
\hline 196 & 1.123512 & & aple 35 \\
\hline 197 & 1.154483 & 51 & 1.134412 \\
\hline 202 & 1.109303 & 97 & 1.137997 \\
\hline 203 & 1.082437 & 131 & 1.137179 \\
\hline 203 & 1.127768 & 183 & 1.137990 \\
\hline 204 & 1.148733 & 184 & 1.128744 \\
\hline & aple 26 & 196 & 1.059516 \\
\hline 51 & 1.115073 & 197 & 1.133449 \\
\hline 54 & 1.123056 & 202 & 1.132267 \\
\hline 97 & 1.125539 & 203 & 1.125174 \\
\hline 131 & 1.147686 & 204 & 1.109277 \\
\hline 144 & 1.139263 & 205 & 1.057631 \\
\hline 161 & 1.147373 & 144 & 1.121594 \\
\hline 183 & 1.157010 & & ple 36 \\
\hline 184 & 1.031963 & 54 & 1.074576 \\
\hline 196 & 1.154371 & 97 & 1.036314 \\
\hline 197 & 1.059236 & 131 & 1.053430 \\
\hline 202 & 1.152848 & 161 & 1.094330 \\
\hline 203 & 1.130625 & 183 & 1.116868 \\
\hline 204 & 1.025311 & 196 & 1.125180 \\
\hline & pple 27 & 197 & 1.082492 \\
\hline 51 & 1.096225 & 202 & 1.170727 \\
\hline 54 & 1.122555 & 203 & 1.135916 \\
\hline 94 & 1.119886 & 204 & 1.090704 \\
\hline 97 & 1.115459 & 144 & 1.131716 \\
\hline 128 & 1.122335 & & aple 37 \\
\hline 131 & 1.140530 & 160 & 1.101106 \\
\hline 144 & 1.114575 & 183 & 1.138975 \\
\hline 160 & 1.117057 & 184 & 1.051029 \\
\hline 161 & 1.154783 & 196 & 1.087405 \\
\hline 183 & 1.084856 & 197 & 1.086041 \\
\hline 184 & 1.062688 & 202 & 1.111035 \\
\hline 196 & 1.116923 & 203 & 1.077545 \\
\hline 197 & 1.089067 & 204 & 1.143120 \\
\hline 202 & 1.131161 & 205 & 1.111031 \\
\hline 203 & 1.111441 & 144 & 1.157282 \\
\hline 204 & 1.070181 & & \\
\hline 205 & 1.044545 & & \\
\hline
\end{tabular}

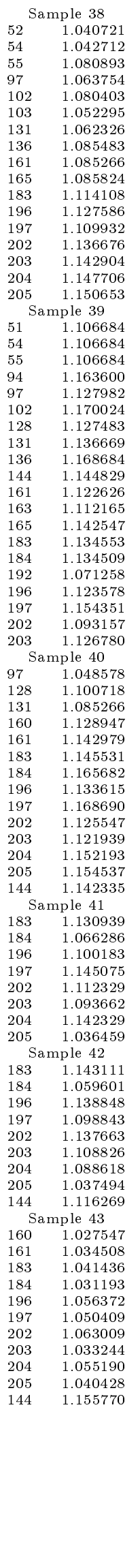

\begin{tabular}{|c|c|c|c|}
\hline & aple 44 & & aple 53 \\
\hline 51 & 1.123430 & 1 & 1.104506 \\
\hline 94 & 1.087655 & 2 & 1.131232 \\
\hline 97 & 1.065137 & 5 & 1.108631 \\
\hline 128 & 1.041334 & 7 & 1.039423 \\
\hline 160 & 1.098552 & 9 & 1.032435 \\
\hline 183 & 1.149181 & 11 & 1.071398 \\
\hline 184 & 1.065275 & 14 & 1.035573 \\
\hline 196 & 1.137259 & 16 & 1.025235 \\
\hline 197 & 1.042784 & 17 & 1.029511 \\
\hline 202 & 1.147155 & 19 & 1.026316 \\
\hline 203 & 1.122248 & 20 & 1.103754 \\
\hline 204 & 1.119005 & 22 & 1.048499 \\
\hline 205 & 1.063995 & 23 & 1.033049 \\
\hline 144 & 1.143685 & 26 & 1.032673 \\
\hline & aple 45 & 28 & 1.028374 \\
\hline 54 & 1.106684 & 30 & 1.027355 \\
\hline 97 & 1.106684 & 31 & 1.025347 \\
\hline 131 & 1.107864 & 33 & 1.037560 \\
\hline 161 & 1.143551 & 37 & 1.026274 \\
\hline 183 & 1.153712 & 39 & 1.031014 \\
\hline 196 & 1.139311 & 41 & 1.029726 \\
\hline 202 & 1.087485 & 42 & 1.025655 \\
\hline 144 & 1.171566 & 45 & 1.090215 \\
\hline & pple 46 & 48 & 1.036179 \\
\hline 183 & 1.093190 & 49 & 1.025337 \\
\hline 202 & 1.150322 & 51 & 1.083776 \\
\hline 203 & 1.143839 & 53 & 1.067530 \\
\hline 204 & 1.134963 & 54 & 1.036694 \\
\hline 205 & 1.101452 & 55 & 1.039484 \\
\hline 144 & 1.153524 & 90 & 1.079569 \\
\hline & aple 47 & 94 & 1.115098 \\
\hline 51 & 1.108163 & 97 & 1.094134 \\
\hline 94 & 1.025390 & 102 & 1.093719 \\
\hline 97 & 1.079345 & 125 & 1.086318 \\
\hline 183 & 1.137268 & 128 & 1.130865 \\
\hline 196 & 1.139571 & 131 & 1.107587 \\
\hline 197 & 1.027613 & 136 & 1.108395 \\
\hline 202 & 1.139727 & 144 & 1.155545 \\
\hline 203 & 1.127481 & 160 & 1.107560 \\
\hline 204 & 1.026141 & 161 & 1.116131 \\
\hline 144 & 1.106449 & 183 & 1.126000 \\
\hline & aple 48 & 184 & 1.129992 \\
\hline 51 & 1.133852 & 192 & 1.112735 \\
\hline 54 & 1.040814 & 196 & 1.139819 \\
\hline 94 & 1.121721 & 197 & 1.143942 \\
\hline 97 & 1.104197 & 202 & 1.157881 \\
\hline 128 & 1.120460 & 203 & 1.152389 \\
\hline 160 & 1.088350 & 204 & 1.155370 \\
\hline 161 & 1.028864 & 205 & 1.154808 \\
\hline 183 & 1.140503 & & aple 54 \\
\hline 184 & 1.129318 & 51 & 1.183945 \\
\hline 196 & 1.133627 & 54 & 1.170834 \\
\hline 197 & 1.136283 & 94 & 1.175686 \\
\hline 202 & 1.146893 & 97 & 1.177040 \\
\hline 203 & 1.131323 & 128 & 1.176144 \\
\hline 204 & 1.143477 & 131 & 1.180882 \\
\hline 205 & 1.103503 & 160 & 1.200567 \\
\hline 144 & 1.122832 & 161 & 1.208631 \\
\hline 144 & 1.148182 & 183 & 1.211733 \\
\hline & hple 49 & 184 & 1.226654 \\
\hline 202 & 1.153009 & 196 & 1.186249 \\
\hline 203 & 1.140358 & 197 & 1.197478 \\
\hline 144 & 1.156699 & 202 & 1.156294 \\
\hline & aple 50 & 203 & 1.151315 \\
\hline 202 & 1.078599 & 204 & 1.172547 \\
\hline 144 & 1.138170 & 205 & 1.169310 \\
\hline & aple 51 & 144 & 1.215858 \\
\hline 97 & 1.092194 & & aple 55 \\
\hline 131 & 1.045328 & 2 & 1.108985 \\
\hline 161 & 1.043983 & 49 & 1.172046 \\
\hline 183 & 1.136953 & 51 & 1.191910 \\
\hline 196 & 1.134601 & 53 & 1.118907 \\
\hline 197 & 1.063124 & 54 & 1.247578 \\
\hline 202 & 1.153494 & 55 & 1.194333 \\
\hline 203 & 1.137882 & 90 & 1.172572 \\
\hline 204 & 1.109141 & 94 & 1.178380 \\
\hline 205 & 1.034284 & 97 & 1.240256 \\
\hline 144 & 1.100556 & 99 & 1. 126112 \\
\hline & ple 52 & 102 & 1.189284 \\
\hline 51 & 1.139804 & 125 & 1.157819 \\
\hline 94 & 1.053043 & 128 & 1.176991 \\
\hline 97 & 1.065724 & 131 & 1.230083 \\
\hline 144 & 1.138676 & 134 & 1.125924 \\
\hline 183 & 1.133623 & 136 & 1.184949 \\
\hline 184 & 1.043346 & 144 & 1.142835 \\
\hline 196 & 1.116704 & 156 & 1.150901 \\
\hline 197 & 1.091930 & 158 & 1.092351 \\
\hline 202 & 1.105248 & 160 & 1.088089 \\
\hline 203 & 1.114387 & 161 & 1.218187 \\
\hline 204 & 1.054445 & 163 & 1.136737 \\
\hline 205 & 1.025115 & 165 & 1.168204 \\
\hline & & 178 & 1.150996 \\
\hline & & 179 & 1.124657 \\
\hline & & 183 & 1.192063 \\
\hline
\end{tabular}

Sample 55 (contd.)

$184 \quad 1.169666$

$187 \quad 1.163685$

$190 \quad 1.140083$

$191 \quad 1.120078$

$197 \quad 1.150032$

$\begin{array}{ll}198 & 1.110193 \\ 200 & 1.164816\end{array}$

$201 \quad 1.132830$

$202 \quad 1.135987$

1.141093

$\begin{array}{ll}203 & 1.120592 \\ 204 & 1.137160\end{array}$

$05 \quad 1.069088$

Sample 56

$\begin{array}{ll}94 & 1.098512 \\ 97 & 1.053302\end{array}$

$128 \quad 1.092076$

$160 \quad 1.032491$

$83 \quad 1.141989$

$\begin{array}{ll}96 & 1.135873 \\ 197 & 1.091193\end{array}$

$\begin{array}{ll}197 & 1.091193 \\ 202 & 1.112652\end{array}$

$203 \quad 1.088172$

$\begin{array}{ll}204 & 1.026140 \\ 144 & 1.160848\end{array}$

Sample 57

$128 \quad 1.111225$

$\begin{array}{ll}31 & 1.127759\end{array}$

$\begin{array}{ll}183 & 1.063298\end{array}$

$184 \quad 1.119635$

$\begin{array}{ll}197 & 1.123937 \\ 202 & 1.069473\end{array}$

$203 \quad 1.114834$

$204 \quad 1.135745$

$144 \quad 1.159974$

Sample 58

$183 \quad 1.058823$

$184 \quad 1.034539$

$\begin{array}{ll}196 & 1.124095 \\ 197 & 1.134569\end{array}$

$202 \quad 1.117503$

$203 \quad 1.116547$

$204 \quad 1.154145$

$144 \quad 1.166588$

Sample 59

$202 \quad 1.150348$

$203 \quad 1.144365$

$144 \quad 1.174654$

Sample 60

$54 \quad 1.106684$

$\begin{array}{ll}101 & 1.129178 \\ 97 & 1.106684\end{array}$

$\begin{array}{ll}97 & 1.106684 \\ 131 & 1.106684\end{array}$

$161 \quad 1.092454$

$\begin{array}{ll}183 & 1.110989 \\ 196 & 1.102676\end{array}$

$197 \quad 1.121422$

$202 \quad 1.145687$

$03 \quad 1.148215$

$04 \quad 1.143746$

$\begin{array}{ll}205 & 1.028221 \\ 144 & 1.170493\end{array}$

Sample 61

$\begin{array}{ll}160 & 1.080463 \\ 161 & 1.048179\end{array}$

$183 \quad 1.060091$

$184 \quad 1.107530$

$\begin{array}{ll}184 & 1.107530 \\ 196 & 1.127442\end{array}$

$197 \quad 1.175508$

$197 \quad 1.183174$

$\begin{array}{ll}202 & 1.249330 \\ 203 & 1.202328\end{array}$

$203 \quad 1.207230$

$203 \quad 1.222996$

$203 \quad 1.213102$

$204 \quad 1.271386$

$\begin{array}{ll}205 & 1.271319 \\ 144 & 1.168559\end{array}$

Table 12: Correction factors for each sample. The left column of each table has the image number and the right column has the correction factor. 


\section{Additional Sources of Error}

- Robot errors

- Based on the manufacturer's specifications and our observations, we estimate that the systematic angular errors are about one degree and the non-systematic errors are on the order of a tenth of a degree.

- Camera positioning error

- Cameras were manually positioned into holders attached to lab floor. This causes the center of image to shift.

- Variable thickness of sample

- This variable thickness is partially accounted for by changing robot calibration parameters.

- Visibility of sample base in some semi-transparent samples

- Specifically, frosted glass, polyester, cotton and the peacock feather were semitransparent and the backing (either cardboard or the wooden sample base painted in matte black) was slightly visible through these samples.

- Global shape of some samples

- Orange peel, tree bark and straw were all not globally planar in shape (Tree bark wasn't flat at all. Orange peel was as flat as we could make it. The bundle of straw was attached by tape at either end giving it some curvature.)

- Spatial uniformity of lamp

- See Section E for a discussion of the spatial uniformity of each lamp.

- Lamp beam not perfectly parallel

- Time-varying illumination

- See Section E for a discussion of the temporal stability of the lamps. The slight decrease in lamp brightness with time has some effect on the calibration results, but since the decrease is quite small the effect is not significant.

- Angle error due to extended flat sample

- Because the sample has an extended area, there is a slight error in the viewing angle away from the center of the sample. 


\section{References}

[1] Jean. M. Bennett and Lars Mattsson, Surface Roughness and Scattering, Optical Society of America, Washington DC, 1989.

[2] M. Born and E. Wolf, Principles of Optics, Pergamon Press, New York, 1959.

[3] P. Bui-Tuong, "Illumination for Computer Generated Pictures," Communications of the ACM, Vol. 18, pp. 311-317, 1975.

[4] S. Chatterjee, "Classification of Natural Textures using Gaussian Markov Random Fields," Markov Random Fields: Theory and Applications, pp. 159-177, Academic Press, Boston, 1993.

[5] K.W. Fleishcer, D.H. Laidlaw, B.L. Currin, and A.." Barr, "Cellular Texture Generation," Proceedings of SIGGRAPH , 1995.

[6] H. Gouraud, "Continous Shading of Curves Surfaces," IEEE Trans. on Computers, pp. 623-629, June 1971.

[7] B.K.P. Horn and M.J. Brooks, Shape from Shading, MIT Press, Cambridge, Mass, 1989.

[8] R.L. Kashyap, "Characterization and Estimation of Two-Dimensional ARMA Models," IEEE Transactions on Information Theory, Vol. IT-30, No. 5, September 1984.

[9] J.J. Koenderink, A.J. van Doorn and M. Stavridi, "Bidirectional reflection distribution function expressed in terms of surface scattering modes," European Conference on Computer Vision, pp. 28-39, 1996.

[10] J.J. Koenderink and A.J. van Doorn, "Illuminance texture due to surface mesostructure," Journal of the Optical Society of America A, Vol. 13 pp. 452-463, 1996.

[11] J. Krumm and S.A. Shafer, "Texture segmentation and shape in the same image," IEEE Conference on Computer Vision, pp. 121-127, 1995.

[12] J.P. Lewis, "Algorithms for Solid Noise Synthesis," Computer Graphics, Vol. 23, No. 3, pp. 263-270, July 1989.

[13] S.K. Nayar, K. Ikeuchi and T. Kanade, "Surface Reflection: Physical and Geometrical Perspectives," IEEE Transactions on Pattern Analysis and Machine Intelligence, Vol. 13, No. 7, pp. 611-634, July 1991.

[14] S.K. Nayar and M. Oren, "Visual appearance of matte surfaces," Science, Vol. 267, pp. 1153-1156, Feb. 1995.

[15] M. Oren and S.K. Nayar, "Generalization of the Lambertian model and implications for machine vision," International Journal of Computer Vision, Vol. 14, pp. 227-251, 1995.

[16] M.A.S. Patel and F.S. Cohen, "Shape from texture using Markov random field models and stereowindows," IEEE Conference on CVPR, pp. 290-305, 1992.

[17] D.R. Peachy, "Solid Texturing of Complex Surfaces," Proceedings of SIGGRAPH 1985, Computer Graphics, Vol. 19, pp. 279-286, 1985.

[18] K. Perlin, “ An Image Synthesizer," Computer Graphics, Vol. 19, No. 3, pp. 287-296, July 1985.

[19] K. Perlin, “ Hypertexture,” Computer Graphics, Vol. 23, No. 3, pp. 253-262, July 1989. 
[20] R.W. Picard, T. Kabir and F. Liu, " Real-time recognition with the entire Brodatz texture database," IEEE Conference on CVPR, pp. 638-9, 1993.

[21] P. Poulin and A. Fournier, "A Model for Anisotropic Reflection," Computer Graphics, Vol. 24, No. 4, August 1990.

[22] G. Sakas and B. Kernke, "Texture Shaping: A Method for Modeling Arbitrarily Shaped Volume Objects in Texture Space," Photorealistic Rendering in Computer Graphics, Proceedings of the Second Eurographics Workshop on Rendering, Springer-Verlag, New York, 1994.

[23] B.J. Super and A.C. Bovik, "Shape from texture using local spectral moments," IEEE Transactions on Pattern Analysis and Machine Intelligence, Vol. 17, pp. 333-343, 1995.

[24] H.D. Tagare and R.J.P. DeFigueiredo, "A Framework for the Construction of Reflectance Maps for Machine Vision," CVGIP: Image Understanding, Vol. 57, No. 3, pp. 265-282, May 1993.

[25] K.E. Torrance and E.M. Sparrow, "Theory for Off-Specular Reflection from Roughened Surfaces," Journal of the Optical Society of America, Vol. 57, No. 9, pp. 1105-1114, 1967.

[26] L. Wang and G. Healey, "Illumination and geometry invariant recognition of texture in color images," IEEE Conference on CVPR, pp. 419-424, 1996.

[27] G.J. Ward, "Measuring and Modeling Anisotropic Reflection," Computer Graphics, Vol. 26, No. 2, ACM SIGGRAPH, pp. 255-263, July 1992.

[28] S.H. Westin, J.R. Arvo, and K.E. Torrance, "Predicting Reflectance Functions from Complex Surfaces," Computer Graphics, Vol. 26, No. 2, ACM SIGGRAPH, pp. 255-263, July 1992.

[29] L.B. Wolff, "A Diffuse Reflectance Model for Smooth Dielectrics," Journal of the Optical Society of America A - Special Issue on Physics Based Machine Vision, Vol. 11, pp. 2956-2968, November 1994.

[30] R.J. Woodham, "Photometric Methods for Determining Surface Orientation from Multiple Images," Optics Engineering, Vol. 19, No. 1, pp. 139-144, 1980.

[31] Z. Xie and M. Brady, " Texture segmentation using local energy in wavelet scale space," ECCV, Vol. 1, pp. 304-313, 1996. 